Aus der Abteilung Klinische Neurophysiologie

(Prof. Dr. med. W. Paulus)

im Zentrum Neurologische Medizin

der Medizinischen Fakultät der Universität Göttingen

in Zusammenarbeit mit dem

Institut für Musikphysiologie und Musikermedizin

(Prof. Dr. med. E. Altenmüller)

der Hochschule für Musik und Theater Hannover

\title{
Kathodale transkranielle Gleichstromstimulation (tDCS) bei Gitarristen mit fokaler Dystonie
}

\author{
INAUGURAL - DISSERTATION \\ zur Erlangung des Doktorgrades \\ der Medizinischen Fakultät \\ der Georg-August-Universität zu Göttingen \\ vorgelegt von \\ Matthias Weidenmüller \\ aus \\ Stadthagen
}

Göttingen 2010 
Dekan: Prof. Dr. med. C. Frömmel

I. Berichterstatter: Prof. Dr. med. M.A. Nitsche

II. Berichterstatter/ in: Priv.-Doz. Dr. med. Dr. rer. nat. B. Zirn

III. Berichterstatter/ in: Prof. Dr. med. M. Oppermann

Tag der mündlichen Prüfung: Dienstag, d. 07.12.2010 


\section{Abkürzungsverzeichnis}

$\begin{array}{ll}\text { ADDS } & \text { Arm Dystonia Disability Scale } \\ \text { ANOVA } & \text { Varianzanalyse (Analysis of Variance) } \\ \text { Botox } & \text { Botulinumtoxin } \\ \text { cAMP } & \text { zyklisches Adenosin-Mono-Phosphat } \\ \text { Cz } & \text { zentraler Punkt entsprechend dem 10-20-System für das EEG } \\ \text { Dyst } & \text { dyston } \\ \text { EEG } & \text { Elektroenzephalogramm } \\ \text { EMG } & \text { Elektromyographie } \\ \text { FD } & \text { fokale Dystonie } \\ \text { GABA } & \text { Gamma-Amino-Buttersäure } \\ \text { Grobm } & \text { grobmotorisch } \\ \text { ICC } & \text { Intraklassenkorrelation } \\ \text { KE } & \text { Kameraeinstellung } \\ \text { Komp } & \text { kompensatorisch } \\ \text { Laut } & \text { Lautstärke } \\ \text { LTD } & \text { Langzeitdepression (long-term depression) } \\ \text { LTP } & \text { Langzeitpotenzierung (long-term potentiation) } \\ \text { M1 } & \text { primär motorischer Kortex } \\ \text { mA } & \text { Milliampere } \\ \text { MCP } & \text { Metakarpophalangeales Gelenk } \\ \text { MEG } & \text { Magnetenzephalographie } \\ \text { MEP } & \text { motorisch evoziertes Potential } \\ \text { MIDI } & \text { musical instrument digital interface } \\ \text { Min } & \text { Minuten } \\ \text { MRT } & \text { Magnetresonanztomographie } \\ \text { NMD } & \text { N-Methyl-D-Aspartat } \\ \text { Reitpunkt unmittelbar nach Stimulation, Sham-Bedingung } \\ \text { Zignifikanzniveau (probability) }\end{array}$


(r)TMS (repetitive) transkranielle Magnetstimulation

SD Standardabweichung (standard deviation)

Sek Sekunden

SICI short-latency intracortical inhibition

SP Zeitpunkt 60 Minuten nach Stimulation, Sham-Bedingung

SR Zeitpunkt 60 Minuten nach Stimulation, Verum-Bedingung

STAI State-Trait-Angstinventar

tDCS transkranielle Gleichstromstimulation (direct current stimulation)

V1 primär visueller Kortex

VP Zeitpunkt vor Stimulation, Sham-Bedingung ( $\mathrm{P}=$ Placebo)

VR Zeitpunkt vor Stimulation, Verum-Bedingung ( $\mathrm{R}=$ Real)

ZNS zentrales Nervensystem 


\section{Inhaltsverzeichnis}

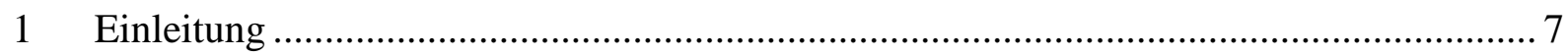

1.1 Die fokale Dystonie, speziell Musikerdystonie................................................... 7

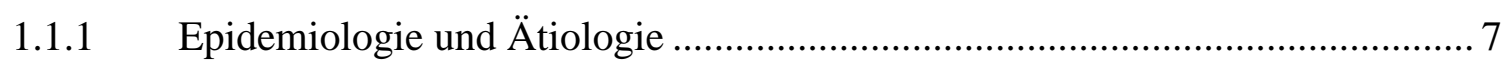

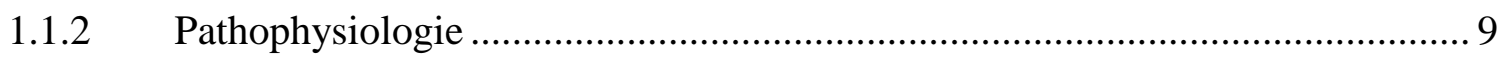

1.1.3 Therapie

1.2 Transkranielle Gleichstromstimulation ............................................................. 14

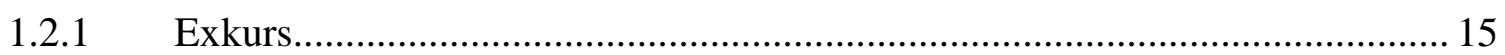

1.2.2 Prinzip und praktische Durchführung der Gleichstromstimulation beim

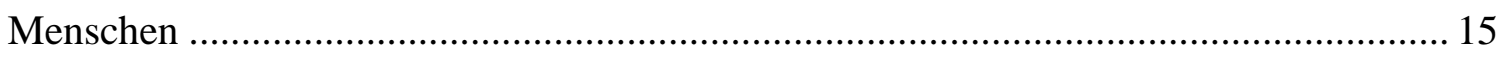

1.2.3 Funktionelle Auswirkungen und klinische Effekte ......................................... 18

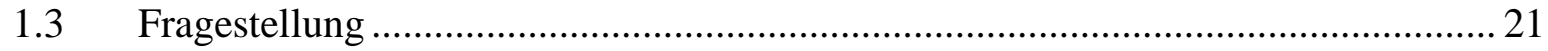

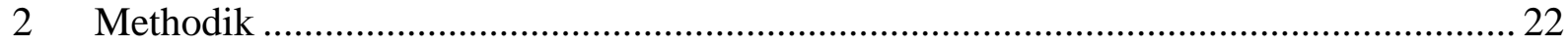

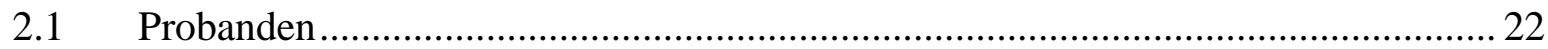

2.1.1 Vorangegangene Therapien (Botox, Trihexyphenidyl) ................................. 23

2.1.2 Kumulative Übezeit................................................................................... 23

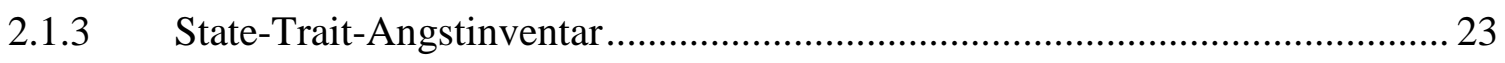

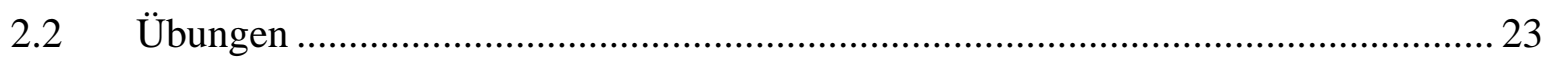

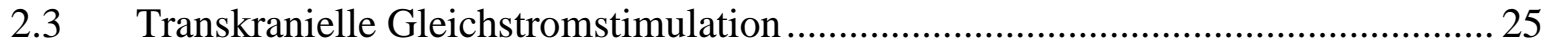

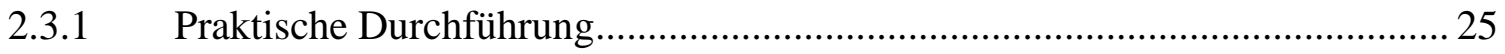

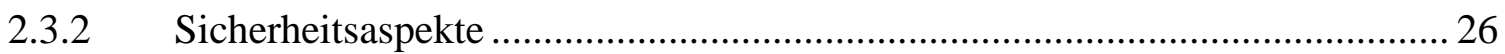

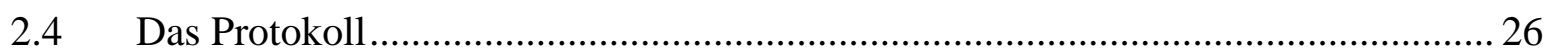

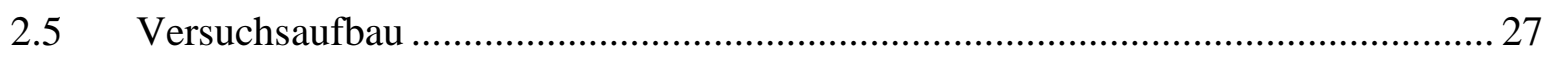

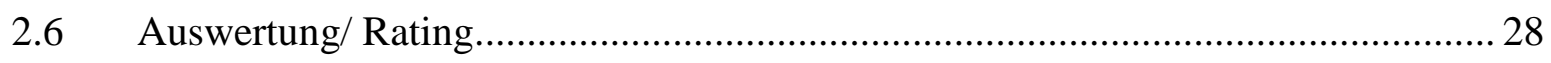

2.6.1 Objektive Einschätzung per Video.............................................................. 28

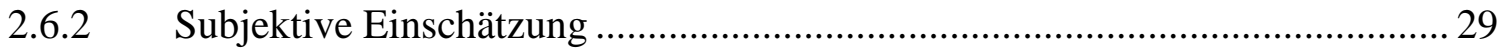

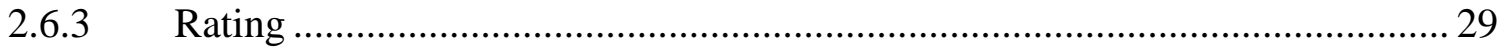

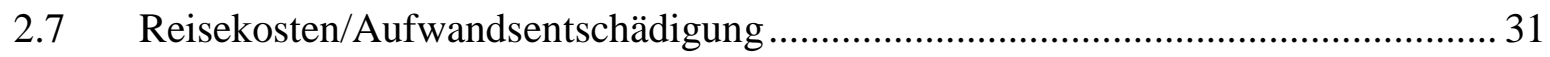

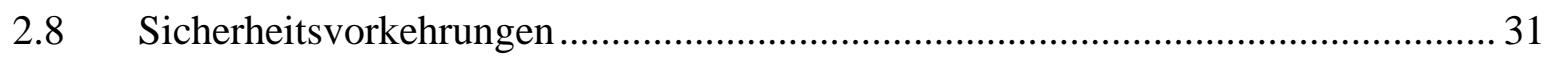

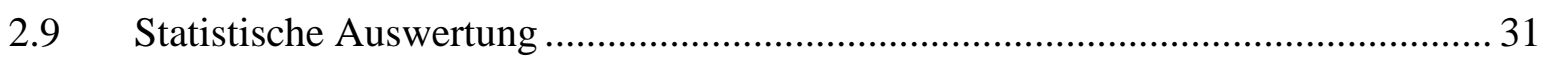




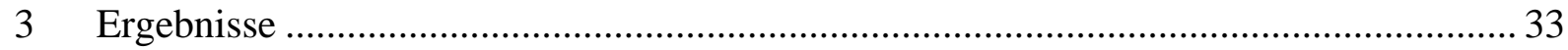

3.1 Effekte kathodaler tDCS über dem primär motorischen Kortex .............................. 33

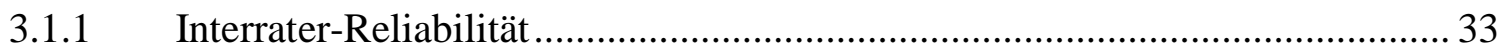

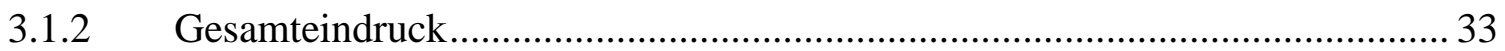

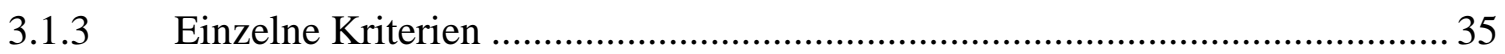

3.1.4 Einzelne Probanden (Proband 1) ....................................................................... 40

3.1.5 Gruppierte Probanden (Erkrankungsdauer der Dystonie) ................................. 41

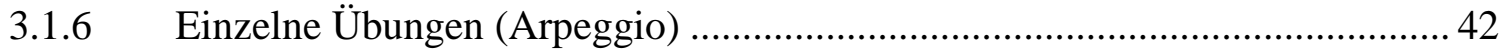

3.1.7 Zusammenfassung der Ergebnisse ............................................................... 45

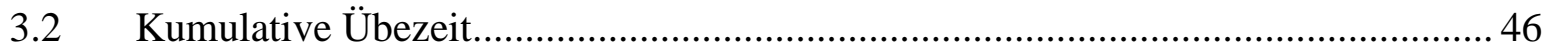

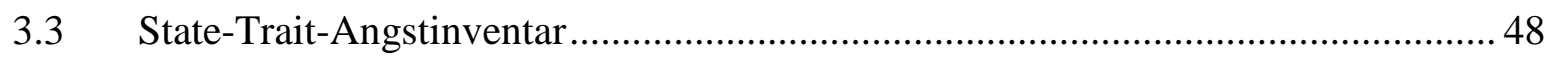

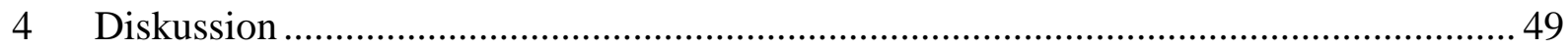

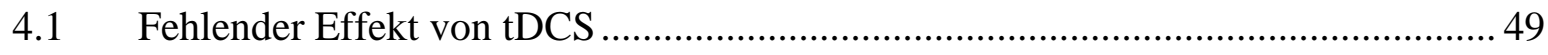

4.2 Verbesserung der Spielfähigkeit bei Proband 1 ..................................................... 52

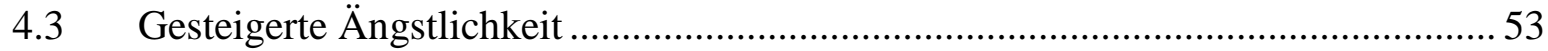

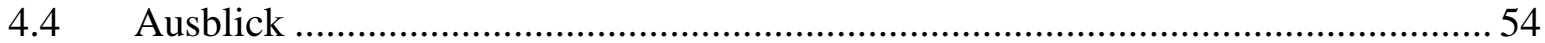

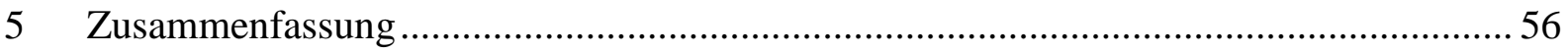

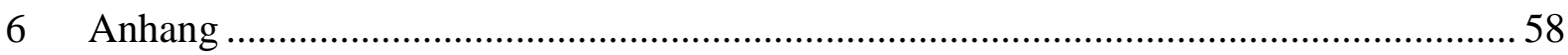

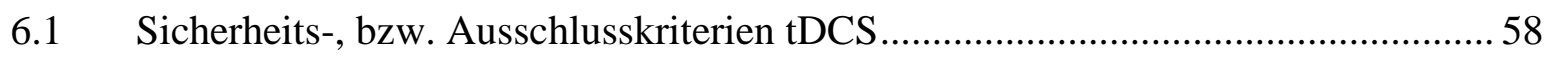

6.2 Edingurgh Handedness Inventory (Oldfield, 1971) ............................................... 59

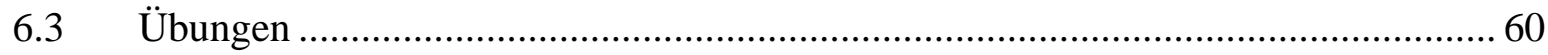

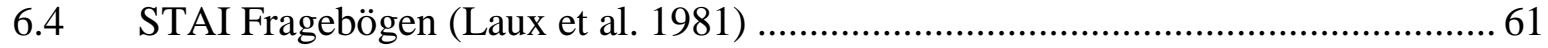

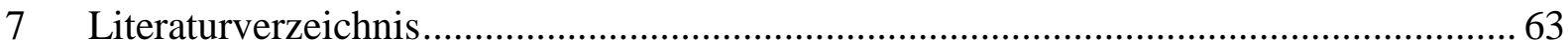




\section{Einleitung}

\subsection{Die fokale Dystonie, speziell Musikerdystonie}

Die fokale Dystonie (FD) ist eine aufgabenspezifische Bewegungseinschränkung. Ist ein Musiker von einer fokalen Dystonie betroffen, so spricht man auch von einer Musikerdystonie. Darunter versteht man eine schmerzlose Beeinträchtigung der Feinmotorik. Der Musiker ist nicht mehr in der Lage, intensiv geübte Bewegungsabläufe flüssig auszuführen. Schnelle Läufe oder Triller, die mitunter auch besonderen Kraftaufwand benötigen, laufen nicht mehr gleichmäßig (Altenmüller 2003). Man kann die Beeinträchtigung häufig unterscheiden in primär dystone und sekundär kompensatorische Bewegungen. So kontrahieren z.B. bei Pianisten oder Gitarristen ungewollt einzelne Finger, bzw. strecken sich einzelne Finger kompensatorisch aus. Für die meisten der betroffenen Musiker ist die Erkrankung sehr einschränkend, so zwingt sie nicht selten den Betroffenen zur Aufgabe der musikalischen Karriere.

\subsubsection{Epidemiologie und Ätiologie}

Verglichen mit der Normalbevölkerung zeigt sich unter Musikern eine deutlich höhere Prävalenz der beschäftigungsabhängigen Dystonie. Liegt die Prävalenz bei anderen Berufsgruppen (z.B. Mikrochirurgen, Uhrmachern, Schreibkräften) bei circa 1:3400, so wird angenommen, dass circa 1\% aller professionellen Musiker vom Musikerkrampf betroffen sind (Altenmüller 2003). Besonders häufig trifft es Männer, im Verhältnis zu Frauen mit einer Häufigkeit von etwa 4:1. Das mittlere Erkrankungsalter liegt in der 4. Lebensdekade (Jabusch und Altenmüller 2006, Lederman 1991, Brandfonbrener und Robson 2002), trifft also die meisten Musiker auf der Höhe ihres Könnens. Pianisten, Holzbläser und Gitarristen, die zusammengenommen etwa 70\% des Patientenkollektivs ausmachen, sind die am häufigsten betroffenen Instrumentalisten (Jabusch und Altenmüller 2006, Brandfonbrener und Robson 2002). Sowohl beim Pianisten als auch beim Gitarristen entwickelt sich die Dystonie in den meisten Fällen in der rechten Hand (Jabusch und Altenmüller 2006). Grund hierfür ist die höhere Spielbelastung als in der linken Hand. 

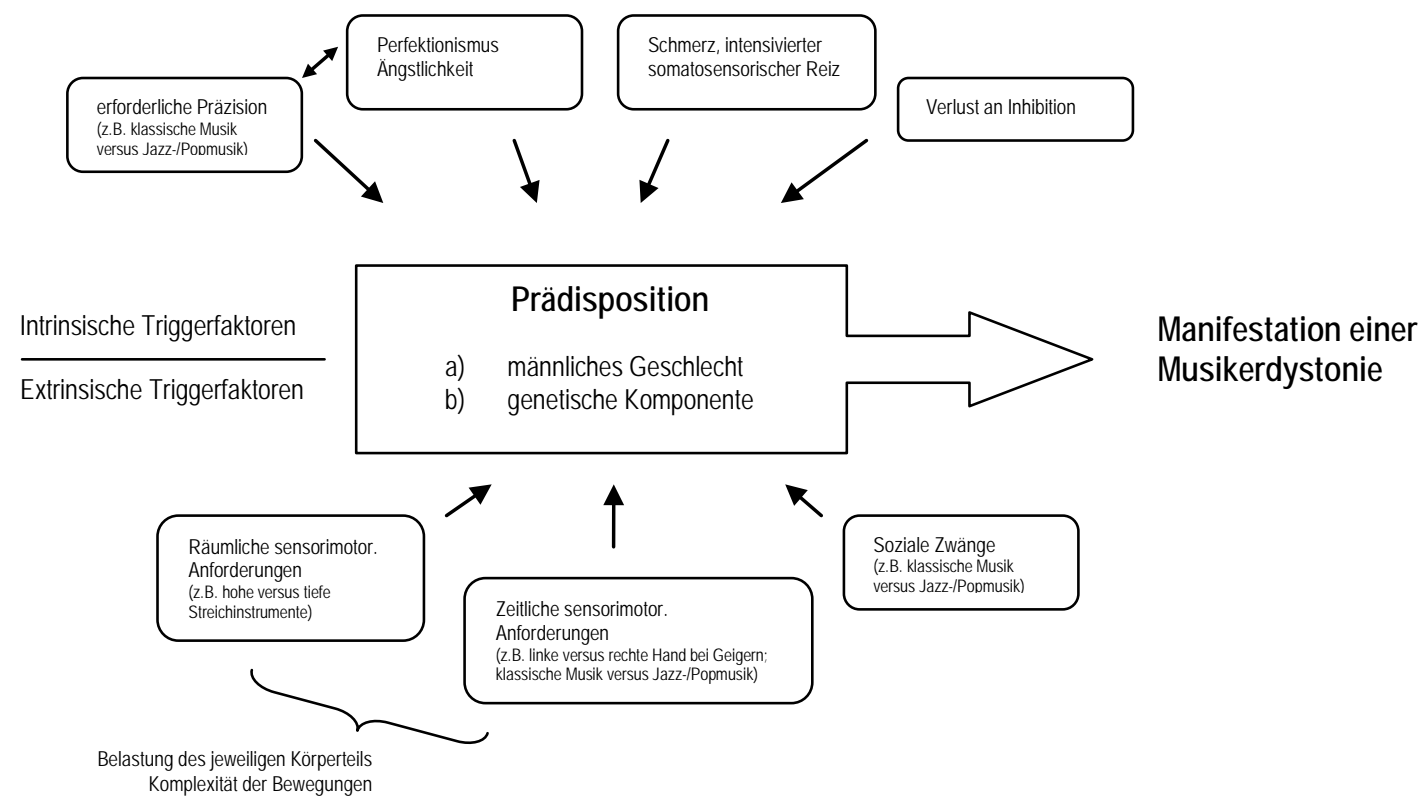

\begin{abstract}
Abbildung 1 Mögliche Interaktionen zwischen Prädisposition und extrinsischen und intrinsischen Faktoren bei der Manifestation einer Musikerdystonie.

(Quelle: modifiziert nach Altenmüller und Jabusch 2009, 152)
\end{abstract}

Die Ätiologie der Musikerdystonie ist multifaktoriell (Jabusch und Altenmüller 2006, s. Abbildung 1). Demnach gibt es neben prädisponierenden Faktoren extrinsische und intrinsische Trigger. Zu den prädisponierenden Faktoren zählt zum einen das männliche Geschlecht, zum anderen eine genetische Komponente. So berichteten circa 6\% in der von ihnen untersuchten Kohorte von einem Fall fokaler Dystonie (Schreibkrampf, bzw. Musikerkrampf) in der Familie (Jabusch und Altenmüller 2006).

Auffällig ist außerdem, dass sich die fokale Dystonie zu 95\% bei Interpreten klassischer Musik entwickelt. Der Grund hierfür liegt in dem besonderen Maß an räumlicher und zeitlicher Präzision, die die Musik dem Interpreten abverlangt (Tubiana und Chamagne 2000, Brandfonbrener und Robson 2002) und als extrinsischer Trigger verstanden werden kann. Neben den besonderen technischen Anforderungen in der klassischen Musik muss der Musiker auch dem Druck einer Aufführung standhalten. Hier ist ein besonders hohes Maß an Konzentration gefordert. Diese Konzentration und Anspannung besteht aus positiven Emotionen, wie der Freude am Musizieren, dem Ausdrücken der in der Musik steckenden Emotionen oder der Bestätigung der eigenen Leistung durch den anschließenden Applaus. Andererseits spielen aber auch negative Emotionen eine Rolle. So z.B. die „Angst des Musikers vor der falschen Note“ oder eine gesteigerte Anforderung an sich selbst durch sehr ausgeprägten Perfektionismus (Jabusch und Altenmüller 2004). Für die Erzeugung und Verarbeitung von Emotionen im menschlichen Gehirn ist vor allem das limbische System verantwortlich (Trepel 2004). Zusammengenommen können diese Emotionen zu einer 
generell gesteigerten Ängstlichkeit des Musikers führen. Diese kann als intrinsischer Trigger eine FD begünstigen. Ebenfalls zu den intrinsischen Triggern kann man physische Störungen zählen. Sie äußern sich in lokalem Schmerz und werden meist durch zu intensives Üben, durch Irritationen peripherer Nerven oder Verletzungen verursacht. Der Schmerz bedingt einen intensivierten somatosensorischen Reiz. Bewegungen werden in Schonhaltung ausgeführt, es können sich unbewusst falsche Bewegungsabläufe einprägen. All dies kann das Entwickeln einer FD begünstigen (Jankovic und Shale 1989, Lederman 1991, Altenmüller 2003). Außerdem taucht ein weiterer intrinsischer Trigger in dem Modell von Jabusch und Altenmüller auf, nämlich ein Mangel an inhibitorischen Schaltkreisen. Auf diesen Punkt werde ich in dem Abschnitt Pathophysiologie näher eingehen.

\subsubsection{Pathophysiologie}

Die Pathophysiologie der Erkrankung ist noch nicht geklärt. Es gibt lediglich verschiedene Ansätze.

Ein Ansatz beschreibt Veränderungen in den Basalganglien. Die Basalganglien regulieren die Motorik. Sie bewirken eine feine Abstimmung aller Bewegungsimpulse, die im Assoziationskortex entworfen wurden. Zu den Basalganglien zählen das Striatum (bestehend aus dem Nucleus caudatus und dem Putamen), der Globus pallidus internus und externus, die Substantia nigra und der Nucleus subthalamicus. Alexander und Crutcher 1990 und später auch Mink 1996 beschreiben den Aufbau der Basalganglien als center-surround Modell mit einem direkten exzitatorischen und einem indirekten inhibitorischen Weg. Der direkte Weg (center) läuft vom Striatum über den Globus pallidus internus zum Thalamus. Dieser Weg besteht aus zwei inhibitorischen GABAergen Synapsen. Eine Hemmung der Hemmung wirkt wie eine Aktivierung des Thalamus (Minus $\times$ Minus $=$ Plus). Beim indirekten Weg (surround) ist neben Striatum und Globus pallidus internus der Nucleus subthalamicus zwischengeschaltet. Insgesamt wirkt dieser Weg bewegungshemmend. Das Prinzip des center-surround Modells ist, dass bei einer spezifischen Bewegung, die über den direkten Weg gebahnt wird, gleichzeitig andere nicht geeignete Bewegungen über den indirekten Weg unterdrückt werden (surround inhibition, s.u.).

Bis zur tatsächlichen Ausführung einer Bewegung sind also viele Hirnareale an dem Zustandekommen dieser Bewegung beteiligt. Neben dem primär motorischen Kortex sind außerdem sekundär motorische Areale involviert, zu denen der prä- und supplementärmotorische Kortex, sowie der cingulär motorische Kortex zählen (Altenmüller

2003). So wird die Bewegung im prämotorischen Kortex geplant, im supplementärmotorischen Kortex wird bei komplexeren Abläufen z. B. das Zusammenspiel 
beider Hände koordiniert. Der cingulär motorische Kortex ist eng mit dem limbischen System verknüpft, das, wie bereits erwähnt, für die Verarbeitung von Emotionen verantwortlich ist. Bevor diese Bewegungsimpulse aus den sekundär motorischen Kortizes jetzt aber über den Thalamus an den primär motorischen Kortex und dann weiter an die Motoneurone zur tatsächlichen Ausführung der Bewegung durch die Muskeln weitergeschickt werden, bedarf es erst einer Rückmeldung aus den Basalganglien und dem Kleinhirn. Diese stehen ebenfalls mit dem Thalamus in Verbindung. In der Zwischenzeit befindet sich der Bewegungsimpuls in einer kortiko-thalamo-kortikalen Warteschleife. Das Kleinhirn ist für feinmotorische Abstimmungen und den richtigen Ablauf einzelner Bewegungen hintereinander verantwortlich. Liegen hier Störungen vor, resultiert dies meist in einer Ataxie. Die Basalganglien funktionieren entweder bahnend oder hemmend. Sie entscheiden, ob eine Bewegung sinnvoll und situationsgerecht ist. Sie sind unabdingbar für jede Art von willkürlicher Bewegung. Sie steuern Ausmaß, Richtung, Kraft und Geschwindigkeit einer Bewegung (Trepel 2004). Liegen in ihnen Veränderungen vor, resultiert dies in Hypo- bzw. Hyperkinesien, zu denen auch der Formenkreis der Dystonien zählt.

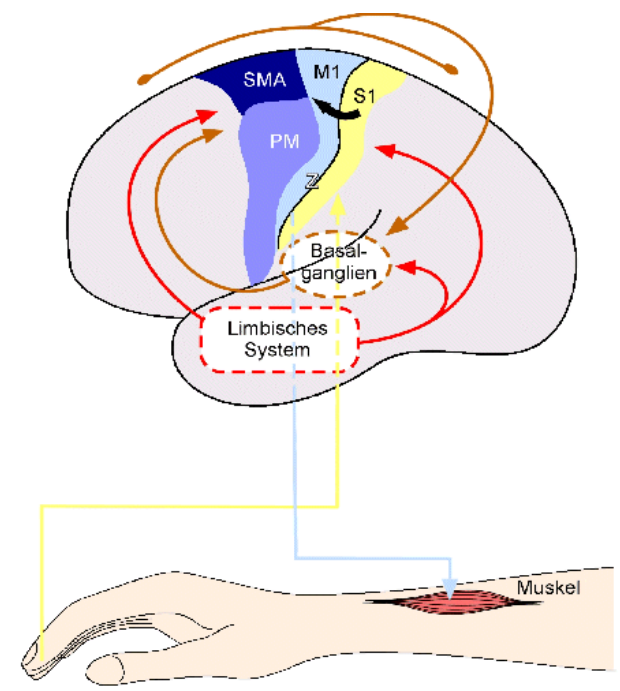

Abblidung 2 Sensomotorik, limbisches System und die Basalganglien stehen in Verbindung und beeinflussen einander (Quelle: Altenmüller 2003, 535)

Eine andere Theorie geht von verminderter kortikaler Inhibition aus. Dystonien werden durch exzessive inadäquate Bewegungen charakterisiert. Es finden exzessive Kokontraktionen der antagonistischen Muskeln statt und es kommt zu einer überflüssigen Überaktivierung der für die Bewegung irrelevanten Muskeln. Diese exzessiven Bewegungen können durch vermehrte Erregung oder verminderte Inhibition entstehen. Das ZNS ist dafür verantwortlich, Erregung und Inhibition auszubalancieren. Es gibt mehr und mehr Beweise dafür, dass bei der Dystonie 
ein Verlust der Inhibition stattfindet (Hallett 2004). Die vermehrte Kokontraktion der antagonistischen Muskeln kommt durch eine verminderte reziproke Hemmung zustande. Das bedeutet, dass z.B. bei Beugen des Armes durch Innervation des M. biceps brachii physiologischerweise auch gleichzeitig der M. triceps brachii gehemmt wird. Ist diese reziproke Hemmung vermindert, ist es wie gleichzeitiges Treten von „Gas und Bremse“. Festgestellt wurde dieser Vorgang erstmals 1983 von Rothwell und Mitarbeitern. Valls-Solé und Hallett veranschaulichten 1995 in einer Studie ebenfalls die verminderte reziproke Hemmung. Bei anhaltender Kontraktion der Flexoren des Handgelenks stimulierten sie gleichzeitig den N. radialis und maßen die Stärke der Kontraktionen der Extensoren per EMG. Diese waren bei Dystonikern deutlich stärker.

Ein von Hallett postuliertes Konzept (Hallett 2004) für diese mangelnde Inhibition stellt die surround inhibition dar. Es stammt ursprünglich aus der sensorischen Physiologie. Auf das visuelle System übertragen, kann man sich das folgendermaßen vorstellen: rezeptive Felder in der Sehrinde werden durch Licht, das in ihr Zentrum fällt, aktiviert; durch Licht, das in deren Peripherie fällt, gehemmt. Dadurch werden Grenzen schärfer dargestellt und Muster und Objekte können entstehen. Überträgt man dieses Modell auf motorische Abläufe, würde bei einer Bewegung das Gehirn die erwünschte spezifische Bewegung bahnen und gleichzeitig andere mögliche Bewegungen unterdrücken. Das Unterdrücken von anderen ungewollten Bewegungen wäre die surround inhibition und ermöglicht so präzisere Bewegungen. Geht man nun von einem Mangel an surround inhibition aus, ließen sich die dystonen Bewegungen gut erklären.

Verschiedene Studien zeigten eine Übererregbarkeit des motorischen Kortex bei FD. Die wahrscheinlichste Erklärung hierfür ist wieder ein Verlust der Inhibition. Ikoma und Mitarbeiter (Ikoma et al. 1996) und Ridding und Mitarbeiter (Ridding et al. 1995) haben dies durch transkranielle Magnetstimulation (TMS) veranschaulicht, durch die motorisch evozierte Potentiale (MEPs) induziert werden können. Ridding und Mitarbeiter zeigten sogar, dass bei Patienten mit fokaler Dystonie die Inhibition in beiden Hemisphären vermindert ist.

Auch der Einfluss von Neurotransmittern auf eine verminderte Inhibition wurde untersucht. So konnten Matsumura und Mitarbeiter (Matsumura et al. 1991) der Dystonie sehr ähnliche Störungen der Motorik an Primaten induzieren. Sie applizierten den Tieren den GABAAntagonisten Bicuculline. GABA als wichtigster hemmender Neurotransmitter im ZNS wurde dadurch geblockt.

Neben der beeinträchtigten Motorik gibt es auch immer mehr Beweise für eine Beeinträchtigung der Sensibilität. Zum einen können wir über feine Rezeptoren in der Haut 
(epikritische Sensibilität) die Umgebung ertasten, zum anderen bekommt das Gehirn über Rezeptoren in Muskeln und Sehnen (Propriozeption) Rückmeldung über Lage und Stellung der Körperteile und die Kraft, die bei Bewegungen aufbegracht wird. Dieses Feedback ist für die Feinabstimmung bei allen Arten von Bewegungen nötig. Es gibt immer mehr Beweise dafür, dass dieses sensible Feedback bei der FD gestört ist. Auf dem primären somatosensiblen Kortex laufen die taktilen Informationen zusammen. Die Nerven unterliegen einer bestimmten Somatotopik. Sie bilden den Körper mit seinen einzelnen Körperteilen auf dem Kortex ab, dem Homunculus. Besonders gut mit Nervenendigungen versorgte Gebiete, wie z.B. das Gesicht oder die Hände, sind größer repräsentiert. Ein rezeptives Feld ist eine Einheit, in der z. B. auf einem Hautfeld Informationen gesammelt und über einen Nerven an das Gehirn weitergeleitet werden. Hier auf dem somatosensiblen Kortex stellt es sich als repräsentatives Areal dar. Je kleiner die rezeptiven Felder, desto besser ist die taktile Diskriminierung in diesem Bereich. Dies ist z. B. besonders an den Fingerkuppen der Fall. In mehreren Studien konnte man nachweisen, dass diese repräsentativen Areale einzelner Finger auf dem somatosensiblen Kortex bei der FD miteinander verschmelzen. In einem Tierexperiment (Byl et al. 1996) ließ man zwei Affen über Wochen hinweg tausendemal die Hand öffnen und schließen. Anschließend stellte man die rezeptiven Felder der Finger mit ihren korrespondierenden repräsentativen Arealen auf dem somatosensiblen Kortex dar. Die Messungen ergaben, dass sich die rezeptiven Felder enorm vergrößert, teilweise sogar über mehrere Finger ausgedehnt hatten. Außerdem waren die einzelnen repräsentativen Areale nicht mehr klar von einander zu trennen. Sie waren verschmolzen. In einer weiteren Studie wurden mittels Magnetenzephalographie (MEG) die rezeptiven Felder der Finger dargestellt (Elbert et al. 1998). Die Fingerspitzen wurden hierbei durch leichten Druck stimuliert. Im Vergleich zu gesunden Probanden war der Abstand der repräsentativen Areale der einzelnen Finger auf dem somatosensiblen Kortex bei den Probanden mit FD verringert. Bara-Jimenez und Mitarbeiter (Bara-Jimenez et al. 1998) zeigten in einer ihrer Studien, dass neben der Annäherung einzelner repräsentativer Areale auch deren Lage entgegen der üblichen strengen Somatotopik eher zufällig ist. Normalerweise ist der Daumen lateral-inferior vom Kleinfinger auf dem sensiblen Kortex dargestellt. Bei 50\% der Probanden mit FD war dies genau umgekehrt. Außerdem war eine zunehmende Lageabweichung mit einer zunehmenden Schwere der Bewegungsstörung zu beobachten. 


\subsubsection{Therapie}

Vorweg muss gesagt werden, dass bis heute keine kurative Therapie der FD bekannt ist. Alle Therapieansätze können nur die Symptomatik verbessern. Es gibt sowohl medikamentöse als auch nicht medikamentöse Optionen.

$\mathrm{Zu}$ den medikamentösen Therapien zählt die lokale Injektion von Botulinumtoxin (z.B. Dysport). Sie wird nur in Fällen empfohlen, in denen primär dystone Bewegungen klar von sekundär kompensatorischen Bewegungen unterschieden werden können. Es ist eines der hochpotentesten Gifte und wirkt an der neuromuskulären Endplatte als Muskelrelaxanz. Es verhindert die Verschmelzung von Transportvesikeln mit der präsynaptischen Membran. Der Neurotransmitter Acetylcholin gelangt so nicht in den synaptischen Spalt, es findet keine Signalübertragung statt, im Prinzip wird eine chemische Denervierung erzeugt. Das Mittel wird in Mikrogramm-Einheiten lokal unter EMG-Monitoring in die von der Dystonie betroffenen Muskeln appliziert. Bei Patienten mit Flexions- oder Extensionsdystonie an einzelnen Fingern sind dies meist die langen Handmuskeln am Unterarm (Jabusch und Altenmüller 2006). Die Wirkung setzt mit einer Verzögerung von 3-5 Tagen ein und hält durchschnittlich über 3 Monate an. Die Gefahr besteht in einer Überdosierung, bei der reversible örtliche Lähmungserscheinungen auftreten können.

Außerdem können zentralwirksame Anticholinergika wie z.B. Trihexyphenidyl (Artane, Parkopan) verabreicht werden. Sie sollen an den Basalganglien wirken. Wenn keine Kontraindikation besteht, sind sie meist Mittel der ersten Wahl. Sie werden einschleichend dosiert, wobei die typischen anticholinergen Nebenwirkungen wie Mundtrockenheit, Müdigkeit, Schwindel etc. auftreten können. Häufig kann die optimale Höhe der Dosierung zur Verbesserung der Symptome nicht erreicht werden. Die Patienten brechen die Therapie aufgrund der starken Nebenwirkungen ab. Prognostische Faktoren für ein gutes Ergebnis bei Behandlung mit Anitcholinergika scheinen zu sein: die Dystonie betrifft den Arm oder die Hand, es wird eine maximal hohe Dosis erreicht und der Patient hat eine geringe Zahl an Begleiterkrankungen, die einer Behandlung bedürfen (Jabusch und Altenmüller 2006).

$\mathrm{Zu}$ den nicht medikamentösen Therapien zählen z. B. ergonomische Hilfen, eine vorübergehende Immobilisierung oder ein Retraining unter pädagogischer Anleitung. Ergonomische Hilfen sollten, wann immer praktizierbar, angewendet werden. Dazu zählen z. B. Schienen für den dystonen Finger oder Veränderungen am Instrument, wie z. B. Verrücken von Klappen bei Blasinstrumenten oder Tragehilfen, wie Gürtel oder Ständer. Mit ihnen wird versucht, dystone Bewegungen zu blockieren oder zu umgehen. Ergonomische Hilfen sind 
allerdings nur effektiv bei Dystonien, die die Extremitäten betreffen (Jabusch und Altenmüller 2006).

Eine weitere Methode ist die vorübergehende Immobilisierung des dystonen Körperteils. Bei betroffenen Pianisten oder Gitarristen wird z. B. der Unterarm mitsamt der Hand über mehrere Wochen geschient. In einer Studie beurteilten Priori und Mitarbeiter (Priori et al. 2001) den Effekt dieser Methode an acht Probanden, die über 4-5 Wochen geschient waren. Eine Woche nach Therapie hatten alle von der Ruhigstellung profitiert. Am Ende einer 24wöchigen Beobachtung des Behandlungserfolges zeigten sich bei 4 Probanden deutliche, bei drei mäßige und bei einem Probanden keine Verbesserung, verglichen mit dem Zeitpunkt vor der Therapie. Es ist eine einfache, relativ effektive und billige Behandlungsmethode.

Es gibt verschiedene Retraining-Möglichkeiten. Sie alle enthalten Elemente folgender Prinzipien: 1. Beschränkung der Bewegung in Kraft und Tempo, um die dystone Bewegung nicht auszulösen, 2. Vermeiden von kompensatorischen Bewegungen und 3. das Bewusstmachen von Bewegungsabläufen. Zum einen gibt es das „Sensory-Motor-Retuning“, bei dem nicht der primär dystone, sondern einer oder mehrere der kompensatorischen Finger ruhig gestellt werden. Candia und Mitarbeiter (Candia et al. 2002) haben dazu eine Studie mit elf Musikern durchgeführt. Acht Tage am Stück mussten die Probanden anderthalb bis zweieinhalb Stunden täglich unter Supervision bestimmte Fingerübungen machen. Anschließend wurden die Probanden gebeten, die Übungen zuhause für ein Jahr täglich eine Stunde fortzuführen. Vor allem für Pianisten und Gitarristen scheint dies eine gute Methode zu sein. Eine andere Methode ist das systematische sensorische Diskriminationstraining nach Byl (Byl und McKenzie 2000). Hierbei wird versucht, die Sensibilität der Finger wieder zu schärfen, indem die Betroffenen das Erkennen von Braille-Schrift (Blindenschrift), Prägungen und Münzen erlernen. Außerdem werden Bewegungsabläufe mit der gesunden Hand vor dem Spiegel geübt. Hierdurch entsteht der Eindruck, dass die Bewegung mit der betroffenen Hand ausgeführt wird.

\subsection{Transkranielle Gleichstromstimulation}

Mit der transkraniellen Gleichstromstimulation (tDCS) ist man in der Lage, Neuroplastizität zu erzeugen. Die Stimulation bewirkt eine Veränderung der Erregbarkeit kortikaler Neurone. Zum einen geschieht dies während der Stimulation durch eine unterschwellige Verschiebung des Ruhemembranpotentials (De- bzw. Hyperpolarisation). Zum anderen resultieren Nacheffekte aus einer Art Langzeitpotenzierung, bzw. -depression. Diese können Minuten bis über eine Stunde anhalten. Über am Kopf angebrachte Elektroden erfolgt die Stimulation 
nicht invasiv und schmerzlos. Je nach Lokalisation der Stimulationselektroden kann mit tDCS Einfluss auf die Motorik oder die Wahrnehmung genommen werden.

\subsubsection{Exkurs}

In den 60er Jahren hat man erste Experimente mit der Gleichstromstimulation an Tieren durchgeführt. Bindman et al. zeigten schon 1964, dass die neuronale Aktivität und Erregbarkeit des sensorimotorischen Kortex durch anodale Stimulation gesteigert und durch kathodale Stimulation vermindert werden kann.

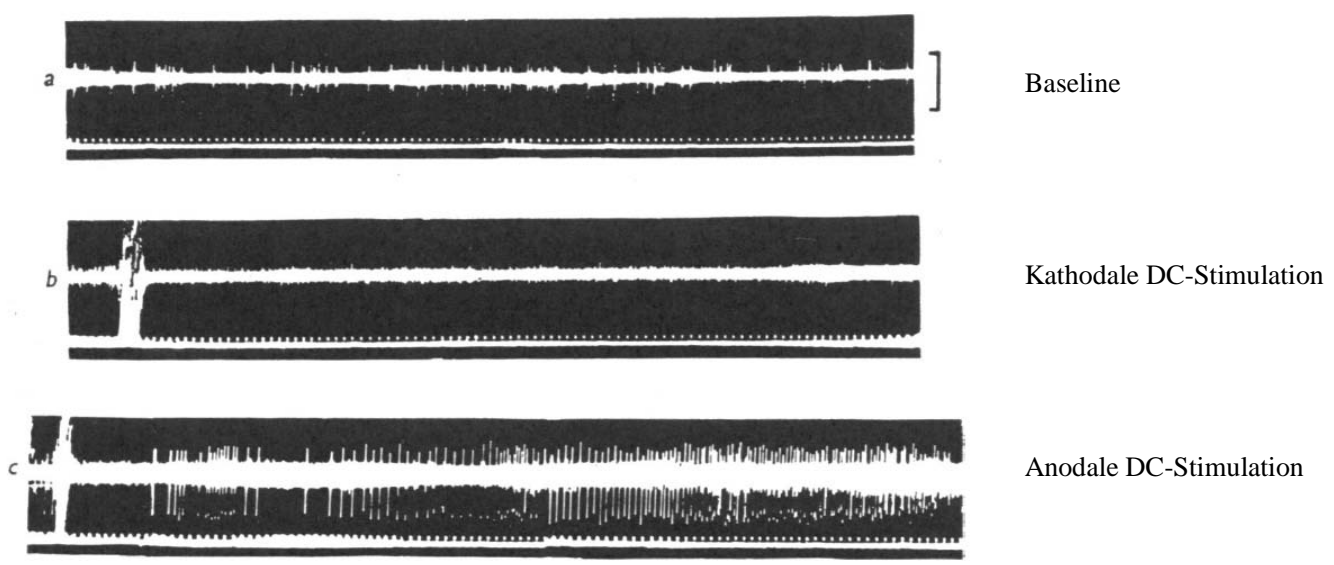

Abbildung 3 intrazerebrale DC-Stimulation bei Ratten, dargestellt sind die abgeleiteten Aktionspotentiale unter den verschiedenen Bedingungen, a) Baseline, b) kathodale DC-Stimulation mit $1 \mu \mathrm{A}$, c) anodale DC-Stimulation mit $0,25 \mu \mathrm{A}$. (Quelle: Bindman et al. 1964, 373)

Damals wurden Versuche an narkotisierten Ratten gemacht, denen Elektroden intrazerebral oder epidural appliziert wurden. Weitere Studien ergaben, dass diese Effekte sowohl durch Veränderungen der intrazellulären Kalzium- und cAMP-Spiegel (Hattori et al. 1990, Islam et al. 1995) bedingt, als auch Proteinsynthese-abhängig sind (Gartside 1968). Nach heutigem Kenntnisstand sind diese Mechanismen der Langzeit-Potenzierung (long-term potentiation (LTP)), bzw. der Langzeit-Depression (long-term depression (LTD)) sehr ähnlich (Hattori et al. 1990, Moriwaki 1991, Islam et al. 1995). Außerdem fand man bereits in den 60ern ebenfalls im Tiermodell heraus, dass auch eine transkranielle Stimulation über oberflächliche Elektroden einen ausreichenden Effekt bringt (Rush und Driscoll 1968). In den 70ern wurde diese Technik an Menschen wiederholt (Dymond et al. 1975).

\subsubsection{Prinzip und praktische Durchführung der Gleichstromstimulation beim Menschen}

Über am Kopf angebrachte Elektroden wird ein geringer Gleichstrom appliziert. Während der Stimulation findet dadurch eine unterschwellige Verschiebung des Ruhemembranpotentials kortikaler Neurone statt. In Abbildung 4 ist die Veränderung kortikaler Erregbarkeit unter tDCS dargstellt. Dabei wurden unter DC-Stimulation mittels TMS motorisch evozierte 
Potentiale (MEPs) erzeugt. Graphisch dargestellt ist die Ableitung der MEPs des Musculus abductor digiti minimi. Je nach Polarität wird bei anodaler Stimulation eine Depolarisation, bei kathodaler Stimulation eine Hyperpolarisation der neuronalen Membran erzeugt (Purpura und McMurtry 1965, Scholfield 1990).

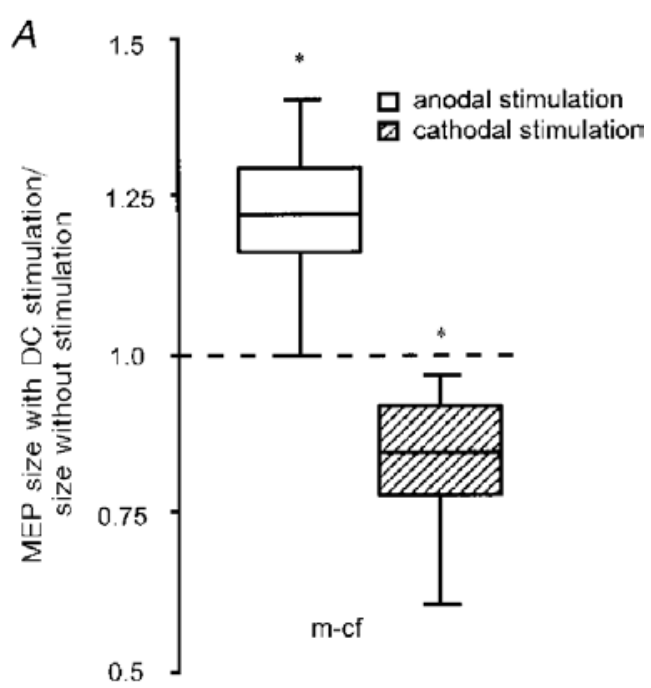

Abbildung 4 Veränderung der kortikalen Erregbarkeit während tDCS, dargestellt sind die MEPs des Musculus abductor digiti minimi, die durch TMS während DC-Stimulation ausgelöst wurden. Entsprechend einem Boxplot enthalten die Kästchen den Bereich 25. bis 75. Perzentile, die Fehlerbalken umfassen die 10. bis 90. Perzentile. Die horizontale Linie in den Kästchen stellt den Median dar. (Quelle: Nitsche und Paulus 2000, 635)

Die Langzeiteffekte nach Stimulation sind NMDA-Rezeptor-abhängig (Nitsche et al. 2003, Nitsche et al 2004a). Außerdem wirken sich adrenerge, dopaminerge und GABAerge Transmitterwege auf die Erregbarkeitsveränderung aus (Nitsche et al. 2004b, Nitsche et al. 2006, Nitsche et al. 2004c).

Der Effekt der Stimulation hängt von Stromdichte, Stromflussrichtung und Stimulationsdauer ab. Die Stromdichte (Stromstärke pro Elektrodenfläche) bestimmt die Stärke der Veränderung des Ruhemembranpotentials (Nitsche und Paulus 2000) und die Stärke des elektrischen Feldes im Kortex. Je größer die Stromdichte, desto tiefer gelegene Kortexschichten werden erreicht. Daneben ist die Stromflussrichtung, in der der Strom durch die entsprechenden Neuronen fließt, entscheidend für den Effekt. Optimal ist der Fluss entlang der Längsachse des Neurons. So kommt es zu einer homogenen Verschiebung des Ruhemembranpotentials (Nitsche und Paulus 2000). Abhängig von der Stimulationsdauer entstehen Nacheffekte oder nicht. Wird nur für wenige Sekunden stimuliert, bleiben Nacheffekte aus. Bei Stimulation über mehrere Minuten resultieren Nacheffekte bis über eine Stunde (Nitsche und Paulus 2000, Nitsche und Paulus 2001, Nitsche et al. 2003). 


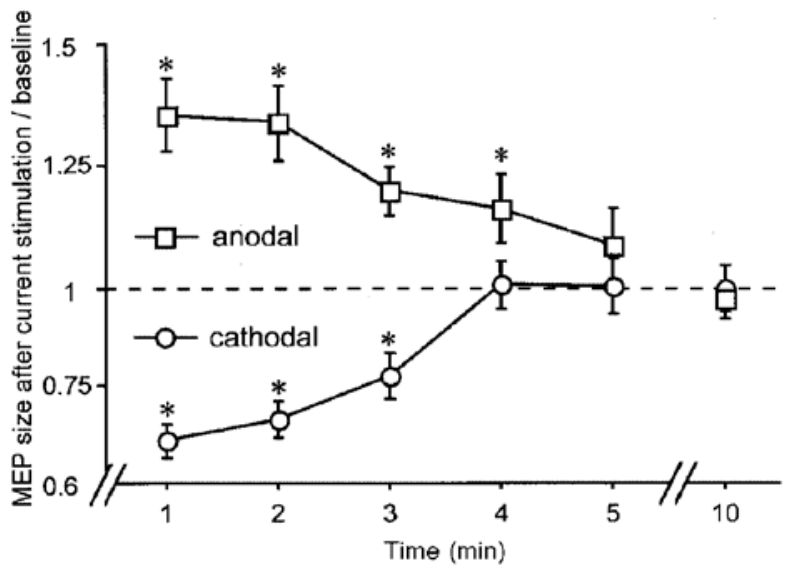

Abbildung 5 tDCS-Nacheffekte abhängig von der Polarität der Stimulation, dargestellt sind die MEPs, die durch TMS ausgelöst wurden, stimuliert wurde über dem primär motorischen Kortex Quelle: Nitsche und Paulus 2000, 636)

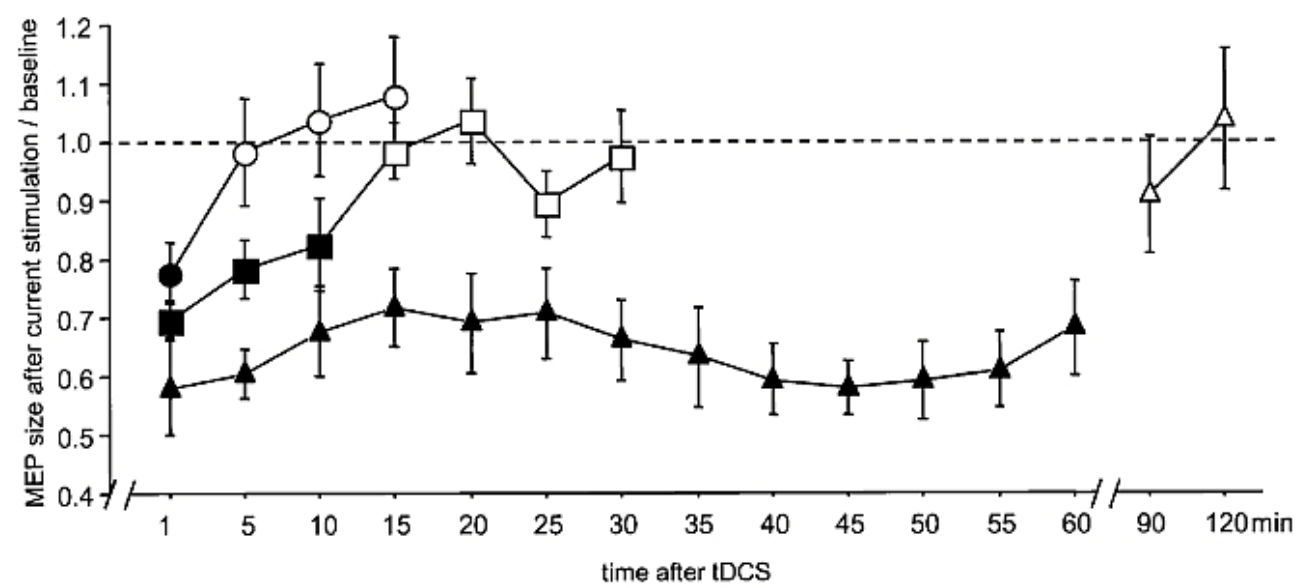

Abbildung 6 Nacheffekte bei kathodaler tDCS, dargestellt sind die MEPs im Zeitverlauf, die durch TMS ausgelöst wurden; stimuliert wurde mit $1 \mathrm{~mA}$ über dem primär motorischen Kortex; $O=5$-minütige tDCS, $\square=7$-miütige tCDS, $\triangle$ = 9-minütige tDCS; ausgefüllte Symbole stellen einen signifikanten Unterschied gegenüber der Baseline dar; (Quelle: Nitsche et al. 2003b, 602)

Technisch ist die tDCS relativ einfach durchzuführen. Es werden zwei großflächige Elektroden mithilfe von Riemen auf der Kopfhaut befestigt. Für besseren Kontakt zur Kopfhaut werden die Elektroden in wassergetränkte taschenartige Schwämme gesteckt. Ein Gleichstromgenerator liefert konstante Stromstärke. Bei Stromstärken von 1-2mA und Elektrodengrößen von 25-35 $\mathrm{cm}^{2}$ kommen entsprechend Stromdichten zwischen 0,03 und $0,08 \mathrm{~mA} / \mathrm{cm}^{2}$ zustande. Stimulationen mit diesen Parametern gelten nach derzeitigem Kenntnisstand als sicher, sofern sie eine Stimulationsdauer von 15-20 Minuten nicht überschreiten (Iyer et al. 2005, Nitsche und Paulus 2001, Nitsche et al. 2003). Es wurden keine ernsthaften Veränderungen im EEG, MRT oder laborchemisch anhand der neuronenspezifischen Enolase (NSE) im Serum, als Marker für Schädigung von Nerven, festgestellt (Nitsche und Paulus 2001, Nitsche et al. 2003). Die Probanden spüren von der 
Stimulation meist nichts, außer einem leichten Jucken auf der Kopfhaut. Missempfindungen treten bei schlechtem Elektrodenkontakt und bei abruptem Stimulationsbeginn bzw. -ende auf. Ein rampenförmiges Ein- und Ausschleichen des Stromflusses soll dies verhindern. Die Elektrodenposition bestimmt die Stromflussrichtung und ist wesentlich für den Effekt. Die Stimulationselektrode wird über dem $\mathrm{zu}$ stimulierenden Kortexareal angebracht. In verschieden Studien wurde eine optimale Position der Referenzelektrode gesucht. So wird sie bei Stimulation des motorischen Kortex am besten kontralateral supraorbital (Nitsche und Paulus 2000), bei Stimulation des visuellen Kortex am besten im Bereich Cz (entsprechend dem 10-20-System für das EEG) angebracht (Antal et al. 2004c). Wie bereits oben erwähnt, ist die Stimulationsdauer entscheidend für den Nacheffekt. Wird z.B. nur für 4 Sekunden mit einer Stromdichte von $0,03 \mathrm{~mA} / \mathrm{cm}^{2}$ stimuliert, so gibt es keinen Nacheffekt. Bei 7-minütiger Stimulationsdauer tritt ein Nacheffekt über wenige Minuten auf. Wird aber anodal 13 Minuten oder kathodal 9 Minuten stimuliert (s. Abbildung 6), so halten Nacheffekte bis über eine Stunde an (Nitsche und Paulus 2000, Nitsche und Paulus 2001). Zwischen den einzelnen Stimulationssitzungen sollte mindestens ein Zeitraum von einer Woche liegen, um eventuelle Überlagerungen von Nacheffekten zu vermeiden. TDCS ist ein günstiges Verfahren für eine Placebo-Stimulation. Dabei kann der Stromfluss rampenförmig ein- und ausgeschlichen werden, wobei die Stimulation nach 30 Sekunden endet. Der Proband empfindet unter Umständen zu Beginn wie bei der Verum-Stimulation ein leichtes Jucken auf der Kopfhaut. Durch das rampenförmige Ein- und Ausschleichen, werden, wie oben erwähnt, Missempfindungen, die bei abruptem Stimulationsbeginn bzw. -ende entstehen, vermieden. Solch eine kurze Stimulationsdauer mit sehr niedriger Stromstärke hat keinen die Stimulation überdauernden Effekt auf die neuronale Erregbarkeit.

\subsubsection{Funktionelle Auswirkungen und klinische Effekte}

In verschiedenen Experimenten untersuchte man den Einfluss von tDCS auf den somatosensorischen und -motorischen Kortex. So betrachteten Matsunaga und Mitarbeiter (Matsunaga et al. 2004) die Veränderung von somatosensorisch evozierten Potentialen (SEPs) unter anodaler und kathodaler Stimulation über dem primär motorischen Kortex. Der somatosensorische Reiz wurde am Nervus medianus gesetzt. Resultat war eine Anhebung der Amplituden der Reizweiterleitung unter anodaler tDCS für circa eine Stunde. Eine kathodale Stimulation hatte keine Veränderung zur Folge. Die transkranielle DCS stellte sich als nützliche Methode heraus, um Plastitzität in der somatosensorischen Reizverarbeitung zu erzeugen. 
In einer anderen Arbeit untersuchte man die taktile Diskriminierung unter tDCS (Rogalewski et al. 2004). Die Probanden sollten die Frequenz einer vibrierenden Sonde beurteilen. Das Resultat war, dass unter kathodaler tDCS über dem somatosensorischen Kortex C4 die taktile Diskriminierung vermindert ist.

Neben somatosensiblen Veränderungen unter tDCS untersuchte man auch Effekte auf motorisches Lernen. Ein Beispiel hierfür ist ein standardisierter Test zur Bestimmung des Lernens einer motorischen Sequenz, die sogenannte Serial Reaction Time Task. Stimuliert wurden der primär motorische, der prämotorische und der präfrontale Kortex. Es zeigte sich ein verbessertes Lernen unter anodaler tDCS über dem primär motorischen Kortex (Nitsche et al. 2003).

Zu vergleichbaren Ergebnissen kam eine Studie von Antal et al. (Antal et al. 2004a), in der es um visuo-motorisches Lernen ging. Probanden mussten auf einem Bildschirm bestimmte Punkte verfolgen und entsprechend mit einer Apparatur die Bewegung nachahmen. Stimuliert wurde neben dem primär motorischen Kortex M1 über V5, einem extrastriatalen visuellen Hirnareal, das stark in der Wahrnehmung von Bewegung involviert ist. Ergebnis war, dass anodale tDCS sowohl über V5 als auch M1 vor allem in der frühen Phase des Lernens dieser visuo-motorischen Aufgabe einen begünstigenden Effekt hatte. Eine Folgestudie über visuomotorisches Lernen (Antal et al. 2004b) ergab unter kathodaler tDCS über V5 einen begünstigenden Effekt, wenn die Aufgabe bereits „überlernt“ war. Als Grund für diese unterschiedlichen Effekte in den unterschiedlichen Phasen des Lernens wird eine PhasenSpezifität der Veränderungen von Neuroplastizität vermutet: eine Steigerung der Erregbarkeit während der frühen Phase des motorischen Lernens soll das Knüpfen von Aufgabenrelevanten Synapsen steigern, wenn eine Aufgabe aber bereits „überlernt“ ist, ist es von Vorteil, Aufgaben-irrelevante Synapsen zu unterdrücken und dadurch die Aufgabenrelevanten neuronalen Verbindungen zu schärfen. Letzteres entspricht auch dem Modell der surround-inhibition.

Klinisch kann tDCS zum Verständnis von Pathomechanismen bei neurologischen und psychischen Erkrankungen beitragen. Darüberhinaus kann es möglicherweise auch von therapeutischem Nutzen sein.

Einige Erfahrungen wurden bereits in der klinischen Anwendung von tDCS bei SchlaganfallPatienten gesammelt. Viele dieser Patienten leiden nach dem Schlaganfall unter einer Hemiparese, verursacht durch einen ischämischen Insult oder eine intrazerebrale Blutung. Die motorische Rehabilitation dieser Patienten wird durch ein Phänomen schlecht adaptierter Plastizität erschwert: dem erlernten „nicht Gebrauchen“ der betroffenen Hemisphäre (Liepert 
et al. 1998). Die anhaltende Bewegungsarmut der paretischen Seite vermindert auch die motorische Repräsentation und Erregbarkeit der entsprechenden kontralateralen Hemisphäre. Durch die Unterrepräsentation der betroffenen Hemisphäre wird eine Überrepräsentation der gesunden Hemisphäre begünstigt. Hummel et al. (Hummel et al. 2005) zeigten, das Schlaganfall-Patienten von einer anodalen tDCS über dem primär motorischen Kortex der betroffenen Hemisphäre profitierten. Die motorische Verbesserung wurde anhand des JebsenTaylor-Handfunktionstests evaluiert, der alltägliche Bewegungsabläufe mit der Hand imitiert. Fregni et al. (Fregni et al. 2005) bewiesen, das kathodale tDCS über dem primär motorischen Kortex der gesunden Hemisphäre einen vergleichbar guten Effekt auf die motorische Verbesserung bei Schlaganfall-Patienten bringt.

Auch im Rahmen von Patienten mit fokaler Dystonie gab es bereits Untersuchungen mithilfe von tDCS. Siebner et al. (Siebner et al. 2004) zeigten anhand einer Gruppe gesunder Probanden in einer Studie, in der sie tDCS mit repetitiver TMS kombinierten, dass Veränderungen der Plastizität innerhalb des motorischen Kortex einer Homöostase unterliegen. Sie sorgt dafür, dass sich die neuronale Erregbarkeit innerhalb eines physiologischen Rahmens bewegt. Repetitive TMS ist genauso wie tDCS in der Lage, Veränderungen in der Erregbarkeit von kortikospinalen Neuronen zu erzeugen. Die Probanden wurden in einer ersten Phase mittels tDCS „präkonditioniert“, in einer zweiten Phase schloss sich die rTMS an. Wurde die neuronale Erregbarkeit zu Beginn durch anodale tDCS gesteigert, konnte dieser Effekt durch die anschließende rTMS rückgängig gemacht werden, die erhöhte Erregbarkeit wurde vermindert. Wurde andersherum die neuronale Erregbarkeit zu Beginn durch kathodale tDCS vermindert, wurde hier ebenfalls Effekt durch die anschließende rTMS aufgehoben, indem die Erregbarkeit wieder erhöht wurde. Es fand regelmäßig eine Gegenregulation statt. Eine Folgestudie ging einen Schritt weiter. Quartarone et al. (Quartarone et al. 2005) untersuchten die neuronale Plastizität, die wie eben besprochen im Normalfall einer Homöostase unterliegt, in einer Gruppe von Probanden mit fokaler Dystonie, speziell mit Schreibkrampf. Analog zu Siebner und Mitarbeitern kombinierte man tDCS und rTMS. Bei den Probanden mit Schreibkrampf jedoch zeigten sich schon bei der „Präkonditionierung“ mit tDCS Unterschiede. Zwar konnte unter anodaler tDCS die Erregbarkeit gesteigert werden, eine kathodale tDCS hatte jedoch keinen inhibitorischen Effekt auf die Erregbarkeit. Außerdem zeigte die anschließende rTMS nicht die gleichen Effekte wie zuvor bei den gesunden Probanden. Die durch anodale tDCS gesteigerte Erregbarkeit konnte durch nachfolgende rTMS nicht aufgehoben, bzw. gegenreguliert werden. Man zeigte, dass homöostatische Regelkreise, insbesondere inhibitorische 
Regelkreise, die die neuronale Erregbarkeit in einem physiologischen Rahmen halten, bei Menschen mit Schreibkrampf gestört sind.

\subsection{Fragestellung}

Zusammenfassend hatten wir folgende Überlegungen als Ausgangspunkt für unsere Studie:

Allgemein liegt der Pathophysiologie der FD eine mangelnde Inhibition auf verschiedenen Ebenen im ZNS zugrunde. Zum einen mangelnde Inhibition auf der Ebene des motorischen Kortex, die sich einerseits in einer Übererregbarkeit mit gesteigerten MEPs (Ikoma et al. 1996, Ridding et al. 1995), andererseits in der Kokontraktion von antagonistischen Muskeln (Rothwell et al. 1983, Valls-Solé und Hallett 1995) äußert. Außerdem liegt eine mangelnde surround-inhibition innerhalb der Basalganglien vor (Hallett 2004): gleichzeitiges Bahnen von gewünschten Bewegungen und Unterdrücken von ungeeigneten Bewegungen und somit das Ermöglichen von präziseren Bewegungen funktioniert nicht mehr richtig. Auch die postulierte gestörte Homöostase bei Dystonikern (Siebner et al. 2004, Quartarone et al. 2005), wodurch kortikale Erregbarkeit nicht mehr in einem physiologisch sinnvollen Rahmen gehalten werden kann, geht in die gleiche Richtung.

Die Studie von Antal et al. (Antal et al. 2004) hat außerdem anhand gesunder Probanden gezeigt, dass kathodale tDCS über dem visuellen Kortex V5 (s.o.) beim Ausführen von visuomotorischen Aufgaben in der Lage war, Wahrnehmung und Ausführung zu fokussieren. Dies versuchte man dadurch zu erklären, dass durch die kathodale tDCS die globale kortikale Erregbarkeit und damit die Aktivierung von konkurrierenden, bzw. ungeeigneten Bewegungen vermindert wird.

Übertragen auf unsere Studie stellten wir uns die Frage, ob eine kathodale tDCS über dem primär motorischen Kortex bei Gitarristen mit FD (Musikerdystonie) ebenfalls in der Lage ist, gesteigerte kortikale Erregbarkeit (mangelnde Inhibition) zu reduzieren und damit eine fokussiertere neuronale Aktivität zu ermöglichen. Kann kathodale tDCS hier die mangelnde surround-inhibition ausgleichen und dadurch die Koaktivierung von ungeeigneten Bewegungsabläufen vermindern, gewünschte Bewegungen dagegen präzisieren? Werden die Gitarristen von einer solchen Stimulation profitieren und hat sie therapeutischen Nutzen? 


\section{Methodik}

\subsection{Probanden}

Für unsere Studie luden wir zehn männliche Gitarristen ein, die allesamt unter fokaler Dystonie leiden. Das Durchschnittsalter der Probanden betrug 48,8 \pm 6,43 (Standardabweichung) Jahre. Der jüngste Teilnehmer war 39, der älteste 57 Jahre alt. Der Beginn der Erkrankung lag im Mittel 8,75 \pm 9 Jahre zurück. Kürzeste Erkrankungsdauer war ein halbes Jahr, längste 26 Jahre. Alle Probanden hatten eine fokale tätigkeitsspezifische Dystonie der rechten Hand, bzw. des rechten Armes. Die dystonen Bewegungen beim Gitarrespiel betrafen einen oder mehrere Finger, bzw. bei einem Probanden auch den gesamten Unterarm (Proband 1). Außer beim Spiel auf der Gitarre machten sich Symptome der Dystonie bei drei der Probanden beim Schreiben auf einer Computertastatur bemerkbar, drei Probanden waren beim handschriftlichen Schreiben beeinträchtigt, ein Proband hatte Schwierigkeiten beim Auf- und Zuknöpfen von z.B. Hemden und einer beim Schrauben.

Tabelle 1
\begin{tabular}{|c|c|c|c|c|c|}
\hline Proband & $\begin{array}{l}\text { Alter } \\
\text { (Jahre) }\end{array}$ & Geschlecht & $\begin{array}{l}\text { Erkrankungs- } \\
\text { dauer (Jahre) }\end{array}$ & Händigkeit & dystone Bewegungen \\
\hline 1 & 57 & $\mathrm{M}$ & 11 & $\mathrm{R}$ & $\begin{array}{c}\text { A, Hypertonus in UA und } \\
\text { Hand }\end{array}$ \\
\hline 2 & 39 & $\mathrm{M}$ & 9 & $\mathrm{R}$ & $\mathrm{I}$ \\
\hline 3 & 40 & $\mathrm{M}$ & 1 & $\mathrm{R}$ & $\mathrm{M}$ \\
\hline 4 & 57 & $\mathrm{M}$ & 0,5 & $\mathrm{R}$ & $\mathrm{A}, \mathrm{DM}$ \\
\hline 5 & 48 & $\mathrm{M}$ & 18 & $\mathrm{R}$ & $\mathrm{I}$ \\
\hline 6 & 54 & $\mathrm{M}$ & 2 & $\mathrm{R}$ & $\mathrm{I}$ \\
\hline 7 & 48 & $\mathrm{M}$ & 17 & $\mathrm{R}$ & $\mathrm{P}, \mathrm{A}$ \\
\hline 8 & 47 & $\mathrm{M}$ & 2 & $\mathrm{R}$ & $\mathrm{M}, \mathrm{A}$ \\
\hline 9 & 53 & $\mathrm{M}$ & 26 & $\mathrm{R}$ & $\mathrm{P}$ \\
\hline 10 & 45 & $\mathrm{M}$ & 1 & $\mathrm{~L}$ & $\mathrm{M}$ \\
\hline
\end{tabular}

Mithilfe des Edinburgh-Handedness-Inventory (Oldfield 1971, s. Anhang) haben wir die Händigkeit der einzelnen Probanden ermittelt. Durch Angabe, welche Hand, bzw. welches Auge oder welchen Fuß man bei verschiedenen Tätigkeiten bevorzugt, werden hier zwei Werte ermittelt (Lateralisations-Quotient und Decile), mit denen man die Händigkeit festlegen kann. Demnach waren neun der Probanden rechts- und einer linkshändig. Aus Sicherheitsgründen für die Stimulation mit tDCS galten für die Probanden bestimmte Ausschlusskriterien, die die Probanden nicht aufweisen durften (s. Anhang). Für die Studie wurde vorab ein Antrag von der zuständigen Ethikkommission der Georg-August-Universität Göttingen genehmigt (Antragsnummer: 2/3/07), die Studie wurde gemäß den Leitlinien der Deklaration von Helsinki durchgeführt. 


\subsubsection{Vorangegangene Therapien (Botox, Trihexyphenidyl)}

Die Probanden sollten aktuell nicht mit einem zentralwirksamen Anticholinergikum wie z.B. Trihexyphenidyl therapiert werden. Außerdem sollten sie auch nicht in einer Phase sein, in der die Symptomatik unter Botulinumtoxin (Botox)-Therapie verbessert ist. Zwei der Probanden befanden sich in einer Botox-Therapie und verbanden die Teilnahme an der Studie mit einer Botox-Injektion. Es wurde darauf geachtet, dass die Injektion erst anschließend an die Stimulationssitzung erfolgte, um eine Muskelirritation auszuschließen. Die letzte vorangegangene Injektion hatte bei Teilnahme an der Studie ihre Wirkung bereits verloren, sodass die Beeinträchtigung der Spielfähigkeit aufgrund der Dystonie an beiden Terminen vergleichbar war. Ein Proband nahm aufgrund der Dystonie seit mehreren Jahren einen Betablocker (Propanolol), um einem generell erhöhten Muskeltonus entgegenzuwirken.

\subsubsection{Kumulative Übezeit}

Die Probanden mussten Angaben zum Übeverhalten in den einzelnen Lebensabschnitten (010, 11-15, 16-20 Jahre, etc.) machen. Sie sollten die tägliche Übezeit in diesen Abschnitten in Stunden angeben. So konnten wir beurteilen, in welchen Zeiträumen besonders intensiv geübt worden ist.

\subsubsection{State-Trait-Angstinventar}

Neben den Informationen zu der Dystonie selbst haben wir auch die Ängstlichkeit der Probanden abgefragt. Anlass dazu gab die Hypothese, dass auch eine erhöhte Ängstlichkeit bei Musikern ein Faktor für den Ausbruch der Dystonie ist (Jabusch und Altenmüller 2004, Jabusch et al. 2004b). Für eine möglichst objektive Erfassung benutzten wir das State-TraitAngstinventar nach Laux und Mitarbeitern (Laux et al. 1981). Hierbei werden Angst als momentaner Zustand (State) und Ängstlichkeit als generelle Eigenschaft (Trait) unterschieden. Erhöhte Ängstlichkeit (Trait) bewirkt, dass Situationen schneller als bedrohlich eingestuft werden. Es gab einen Fragebogen zur State- und einen zur Trait-Angst. Es wurden jeweils 20 Aussagen getroffen, die von dem Probanden in vier verschieden Abstufungen beantwortet werden konnten (s. Anhang).

\section{2 Übungen}

Gespielt wurden insgesamt 12 standardisierte Übungen (s. Anhang) und ein jeweils für den Probanden individuell schwieriges Stück.

Übung 1 waren Arpeggien: Der Proband spielt gebrochene Akkorde nach definierter Anschlagfolge p-i-m-a-m-i-m-a, wobei der Daumen die Saiten wie folgt wechselt: E-A-D-A. 
Anschließend rutscht die linke Hand, die einen verminderten Akkord greift, einen Bund nach oben. Gespielt wird vom I.-VII. Bund auf- und wieder abwärts.

Die Übungen 2 - 4 bestanden im Anschlagen von leeren Saiten: mit der rechten Hand wurden die einzelnen Saiten (E-D-A-G-H-E, auf- und wieder abwärts) triolisch repetiert. Drei aufeinander folgende Durchläufe wurden mit verschiedener Anschlagfolge gespielt (a-m-i, im-i, a-m-a).

Übungen 5 - 6 waren eine Wiederholung der leeren Saiten mit der Anschlagfolge a-m-i und a-m-a. Dieses mal wurde aber aus einer anderen Kameraperspektive gefilmt (s. u.)

Die Übungen 7 - 9 waren Tonleitern mit Wechselschlag: die linke Hand sollte im V. Bund spielen (B-Dur, Fingersatz vom Fis-Dur Typ), drei Durchläufe wieder mit vorgegebener Anschlagfolge p-i, m-i und a-m.

Die Übung 10 war wieder eine Wiederholung der Tonleiter mit der Anschlagfolge a-m, aus der zweiten Kameraperspektive gefilmt.

Übungen 11 - 12 waren Simultananschlag bzw. geschlossener Anschlag in zwei Varianten: die rechte Hand spielte triolisch, zuerst kleiner Anschlag mit dem Daumen auf der D-Saite, dann mit großem Anschlag mit dem Daumen auf der E-Saite. Die linke Hand sollte einen verminderten Akkord greifen und nach jeder Triole einen Bund weiter rutschen (I.-VII. Bund auf- und wieder abwärts).

Übung 13 bestand aus einer für den Probanden jeweils schwierigen Passage aus seinem Repertoire.

Bei jedem Probanden wurde zu Beginn des 1. Termins für jede einzelne Übung ein Tempo festgelegt, dass für alle Durchläufe an beiden Terminen galt. Die Spielfähigkeit sollte nicht durch unterschiedliche Tempi beeinflusst werden. Je schneller jedoch die Übungen gespielt werden, also je unbewusster, desto eher lassen sich die dystonen Bewegungen provozieren (Altenmüller 2003). Das Gehirn ruft bei schnellem Spiel stark prozeduralisierte Bewegungen, d. h. Bewegungen, die durch langes Üben automatisiert wurden, ab. Andererseits sollte der Gitarrist nicht durch ein für ihn zu schnelles Tempo frustriert werden. Es galt hier, eine Balance durch ein geeignetes Tempo zu finden.

Außerdem haben wir zu Beginn die bevorzugte Anschlagstechnik erfragt. Appoyando ist der angelegte Anschlag, bei dem die Finger auf den Saiten aufliegen. Tirando ist der freie Anschlag, bei dem die Finger vor dem Anschlagen der Saite nicht aufliegen. Angestrebt war der freie Anschlag, da diese Spielweise in den meisten Fällen auch dystone Bewegungen provoziert. Dies war leider nicht bei allen Probanden möglich. Auch hier mussten wir 
abwägen, was dem Probanden zuzumuten war. Deshalb sollte er mit der Technik spielen, die er auch sonst verwendet. Die unterschiedlichen Übungen erforderten ohnehin die unterschiedlichen Anschlagtechniken. So wurde bei der Arpeggio-Übung von den meisten der Tirando-Anschlag verwendet.

\subsection{Transkranielle Gleichstromstimulation}

\subsubsection{Praktische Durchführung}

Die Stimulation erfolgte über zwei $35 \mathrm{~cm}^{2}$ große Elektroden, die zur Herstellung einer besseren Kontaktfläche jeweils in einen wassergetränkten Schwamm gesteckt wurden. Die Elektroden wurden mit Gurten am Schädel fixiert. Die Position der Stimulationselektrode wurde mit einem Maßband ausgemessen (s. Abbildung 8). Zunächst wurde $\mathrm{Cz}$ (entsprechend dem 10-20-System für das EEG) ermittelt. Hierzu wurde die halbe Strecke zwischen Nasion und Inion (Protuberantia occipitalis externa) markiert. Anschließend wurde auf der Linie zwischen den beiden Kiefergelenken und dem markierten Punkt zwischen Nasion und Inion angelegt und ebenfalls die Mitte markiert (Cz). Um die Kiefergelenke einfacher zu ertasten, ließen wir hierzu die Probanden kauen. Von Cz 20\% der Strecke in Richtung linke Hemisphäre befindet sich der primär motorische Kortex (C3 bzw. M1) der rechten Körperhälfte. Die Referenzelektrode wurde kontralateral supraorbital zur Stimulationselektrode, also über dem rechten Auge angebracht.
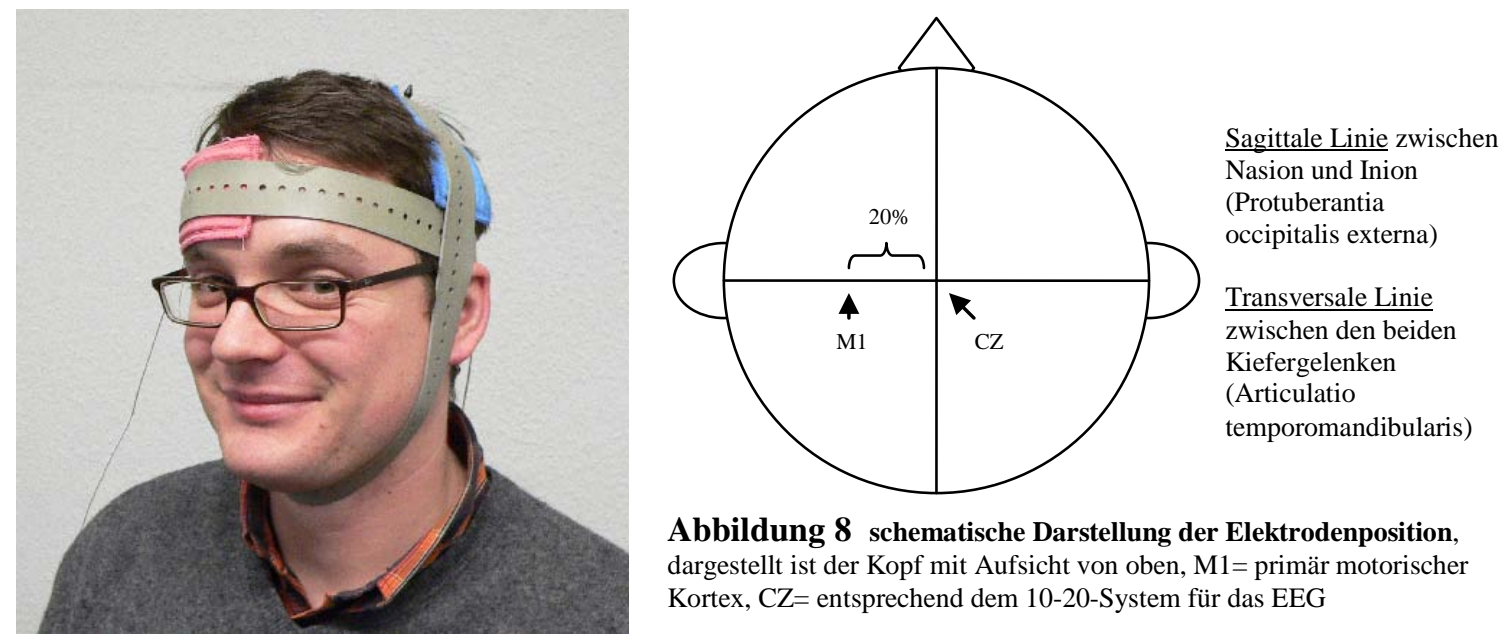

Abbildung 8 schematische Darstellung der Elektrodenposition, dargestellt ist der Kopf mit Aufsicht von oben, M1= primär motorischer Kortex, CZ= entsprechend dem 10-20-System für das EEG

Position der Elektroden

Stimuliert wurde kathodal unter Verum-Bedingungen für 20 Minuten mit $2 m A$, die Stromdichte betrug 0,06 $\mathrm{mA} / \mathrm{cm}^{2}$. Generiert wurde der Strom durch einen batteriebetriebenen Stimulator (DC-Stimulator von der Firma Eldith, Ilmenau, s.o.). Die Stromstärke wurde 
rampenförmig über 8 Sekunden ein- und am Ende wiederum über 8 Sekunden ausgeschlichen, um Missempfindungen zu vermeiden. Unter Placebo-Bedingungen wurde über 30 Sekunden mit 1mA stimuliert, die Stromstärke wurde ebenfalls 8 Sekunden rampenförmig ein- und ausgeschlichen. Die Probanden merkten größtenteils den Stromfluss durch ein leichtes Kribbeln.

\subsubsection{Sicherheitsaspekte}

In einer Studie von Nitsche et al. (Nitsche et al. 2004) konnte anhand von MRT-Bildgebung nachgewiesen werden, dass Stimulation mit einer Stromdichte von 0,03-0,08 mA/cm² (Elektrodengröße 25-35 cm², Stromstärke 1-2mA) über einen Zeitraum von 15-20 Minuten keine Gewebeschäden, wie z.B. Veränderungen an der Blut-Hirn-Schranke oder Ödeme verursacht. Außerdem finden auch keine bedenklichen Überwärmungen unter den Elektroden statt (Nitsche und Paulus 2000). Ein Anstieg der neuronenspezifischen Enolase im Serum, als Marker für neuronale Schäden, konnte ebenfalls nicht beobachtet werden (Nitsche und Paulus 2001, Nitsche et al. 2003). Es war darauf zu achten, dass die Elektroden nicht direkt über Fissuren oder Foraminae im Schädel angebracht wurden, um eine Bündelung des Stroms zu vermeiden (Rush und Discroll 1968). Nach bisherigem Kenntnisstand können unsere Stimulationsparameter also als sicher angesehen werden.

\subsection{Das Protokoll}

Die Probanden kamen an zwei Terminen, mit mindestens einer Woche Abstand. Es wurde einmal Verum-, das andere Mal Placebo-stimuliert. Die Reihenfolge wurde per Los festgelegt. Hierzu wurden zehn Briefumschläge vorbereitet, die die Reihenfolge enthielten (fünfmal Placebo/Verum, fünfmal Verum/Placebo). An jedem Termin wurden sechs Takes (Take = kompletter Durchlauf aller fünf Übungen) aufgenommen: vor der Stimulation, während, unmittelbar nach, 30, 60 und 120 Minuten nach der Stimulation. Dies ist der Zeitraum, in dem erwähnenswerte Nacheffekte auftreten können (Nitsche und Paulus 2001).

Nach jedem Take musste der Proband seine subjektive Selbsteinschätzung in Prozent zu jeder Übung angeben. Hierdurch sollten subjektive Verbesserungen oder Verschlechterungen erfasst werden. Die Spielfähigkeit vor der Stimulation stellte die Baseline dar. Die Angaben wurden in Prozent gemacht, wobei 100\% völlig unbeeinträchtigte Spielfähigkeit bedeutet.

Außerdem haben wir in zwei Fragebögen die Probanden nach ihrer Angst, bzw. Ängstlichkeit befragt (s.o.). Die Fragebögen bestehen aus jeweils 20 Fragen, wobei wir die Angst als Zustand an beiden Terminen, die Angst als Eigenschaft (Ängstlichkeit) nur einmalig beim ersten Termin abgefragt haben. 
In einem persönlichen Fragebogen haben wir wichtige Parameter wie Beginn der Dystonie, die bisher angewandten Therapiemethoden, kumulative Übezeit und Händigkeit erfasst. Bei den Therapiemethoden interessierte uns insbesondere, ob die Probanden bereits zentralwirksam mit Trihexyphenidyl, oder lokal muskelrelaxierend mit Botox behandelt wurden. Ließen sich Probanden Botox spritzen, war wichtig, wann die letzte Injektion erfolgte. Für die Händigkeit (Rechts- oder Linkshänder) gebrauchten wir das Edinburgh Handedness Inventar nach Oldfield (s. Anhang).

\subsection{Versuchsaufbau}

Unsere Gitarristen sollten in einer für sie angenehmen Haltung spielen. Das Spiel wurde aus zwei unterschiedlichen Kameraperspektiven gefilmt (s.u.). Hinter dem Probanden, für ihn nicht einsehbar, war der Stimulator aufgebaut. Dieser stand zusätzlich hinter einem Sichtschutz, so dass auch der Versuchsleiter, der die Selbsteinschätzung und den STAI abzufragen hatte, den Stimulator nicht einsehen konnte.

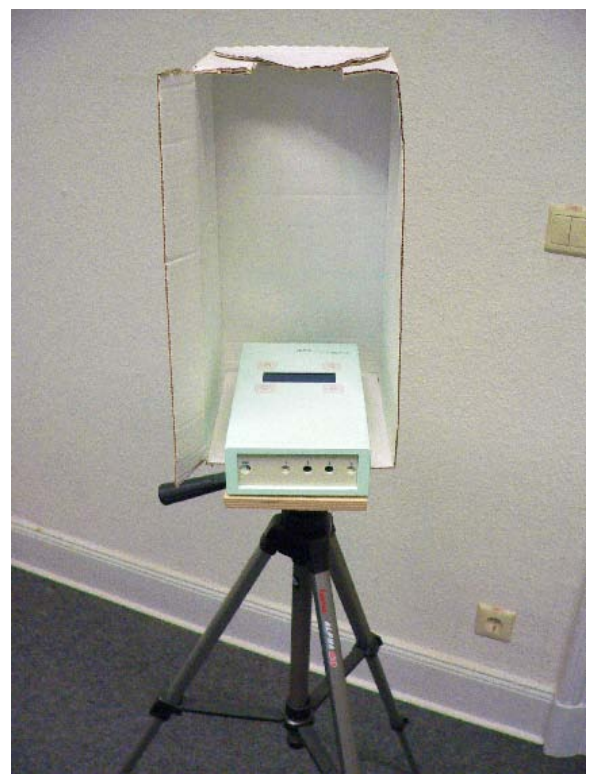

Box mit Stimulator

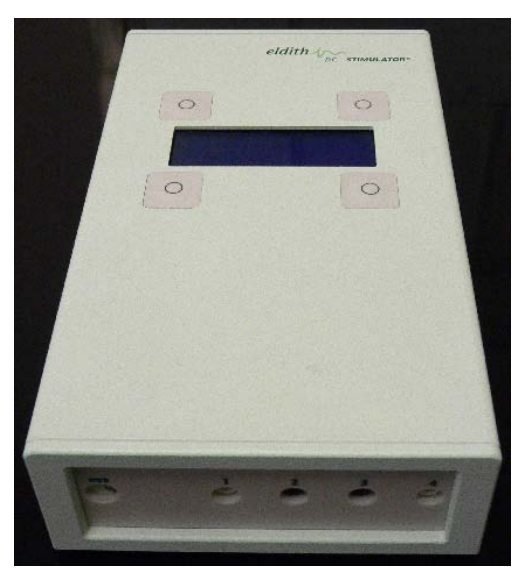

Stimulator

So konnten auch für den Versuchsleiter verblindete Bedingungen geschaffen werden, um mögliche Beeinflussungen beim Abfragen der Spielfähigkeit zu vermeiden. Die Versuche wurden immer mit zwei Personen durchgeführt: dem Versuchsleiter, der die Kamera zu bedienen, STAI und die subjektive Selbsteinschätzung jeweils nach jedem Take abzufragen hatte und einer zweiten Person, die den Umschlag mit der Reihenfolge der beiden Protokolle (Verum/Placebo oder Placebo/Verum) entgegen nahm und den Stimulator bediente. Der Proband wurde über zwei unterschiedliche Stimulationsbedingungen aufgeklärt, wusste aber nicht, dass eine davon eine Placebo-Stimulation ist. 


\subsection{Auswertung/ Rating}

\subsubsection{Objektive Einschätzung per Video}

Alle Probanden wurden während des Spielens gefilmt. Der Fokus lag auf den Bewegungen der rechten Hand. Um diese am besten darzustellen, haben wir aus zwei Kameraeinstellungen gefilmt.

Kameraeinstellung 1 war mit Sicht von oben, hierbei war die Kamera auf einem Stativ montiert. Der Blick fiel entlang des Gitarrenstegs auf die rechte Hand. So zeigten sich Einziehungen oder Streckungen der Finger, vor allem des Zeige- und Mittelfingers am besten. Auch der Daumen war so gut zu beurteilen. Aus dieser Einstellung wurden alle Übungen in allen Anschlagvarianten gefilmt.

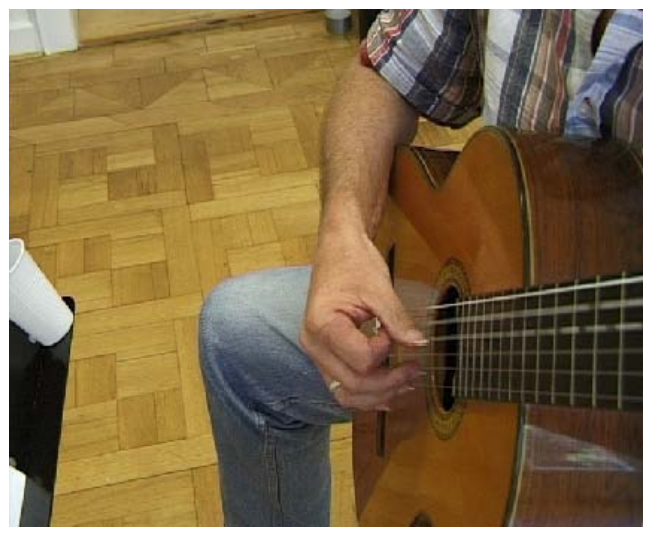

Kameraeinstellung 1

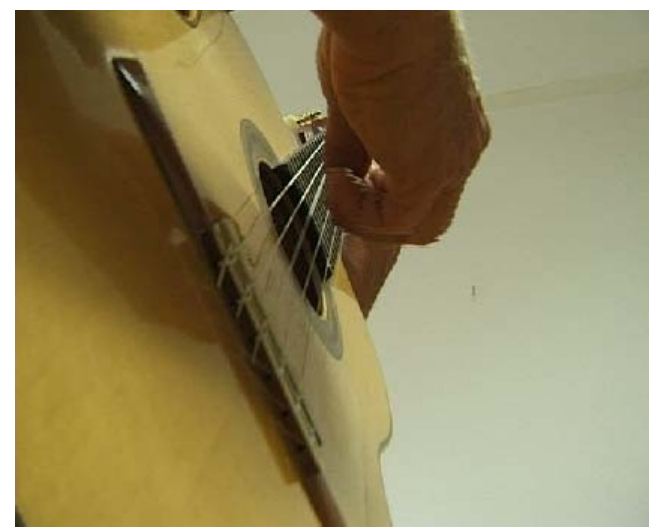

Kameraeinstellung 2

Kameraeinstellung zwei zeigte die Sicht von unten. Aus dieser gegenüberliegenden Position mit Blick von unten auf die rechte Hand konnte man vor allem Ring- und Kleinfinger gut beurteilen. Aus dieser Einstellung wurden Übungen in den Anschlagvarianten gefilmt, bei denen es auf den Ringfinger ankam: Übungen 5 - 6 Leere Saiten (a-m-i und a-m-a) und die Übung 10 Tonleiter a-m. Bei Probanden, bei denen vor allem Ring- und/oder Kleinfinger betroffen sind/ist, haben wir auch das individuell schwierige Stück (Übung 13) aus dieser Einstellung aufgenommen.

Pro Proband wurden 12 Takes aufgenommen (2 Termine mit jeweils 6 Takes (vor, während, nach, 30, 60 und 120 min nach Stimulation)). Zu Beginn jedes Takes haben wir ein Klappenblatt aufgenommen, auf dem die genaue Zuordnung des Takes (Name des Probanden, Datum, Zeitpunkt) stand, z.B.: Herr XY, 28.7.2007, vor Stimulation. So konnte der Take hinterher nach der Videobearbeitung immer genau zugeordnet werden, vor allem zu dem ersten oder zweiten Termin und somit Verum- oder Placebo-Stimulation.

Von jedem Probanden wurde anschließend eine vollständig anonymisierte und randomisierte DVD erstellt. In das Rating flossen nur noch jeweils drei Takes pro Termin ein (vor, 
unmittelbar nach und 60 min nach Stimulation). Grund hierfür war die schon bei diesen drei Takes pro Termin zu beurteilende Datenmenge. Das Filmmaterial von 30 Minuten pro Proband musste wiederholt unter den verschiedenen Kriterien (Lautstärke, Klang, ADDS, etc) betrachtet und beurteilt werden.

\subsubsection{Subjektive Einschätzung}

Nach jedem Take musste der Proband seine subjektive Selbsteinschätzung in Prozent zu jeder Übung angeben. Hierdurch sollten subjektive Verbesserungen oder Verschlechterungen erfasst werden. Die Spielfähigkeit vor der Stimulation stellte die Baseline dar. Die Angaben wurden in Prozent gemacht, wobei 100\% völlig unbeeinträchtigte Spielfähigkeit bedeutet.

\subsubsection{Rating}

Geratet wurde von drei unabhängigen Spezialisten (Prof. Altenmüller, Prof. Kopiecz, Prof. Brendgens). Das Filmmaterial sollte auf bestimmte Kriterien hin beurteilt werden. Die Kriterien waren Rhythmus, Lautstärke, Klang, feinmotorische Spielfähigkeit (Arm Dystonia Disability Scale: ADDS, s.u.), grobmotorisch abnorme Bewegungen und die Anzahl von dystonen bzw. kompensatorischen Bewegung (Frequency of Abnormal Movements: FAM, s.u.). Abschließend sollte für jeden Take ein Gesamteindruck wiedergegeben werden. Abgesehen von der Anzahl der dystonen und kompensatorischen Bewegungen (FAM), sollten die Rater die einzelnen Kriterien auf einer Skala von 0-3 bewerten. Grundlage hierfür war die Arm Dystonia Disability Scale (ADDS: 0= normales Spiel, 1= leichte Schwierigkeiten, 2= moderate Schwierigkeiten, 3= deutliche Schwierigkeiten). Verglichen mit dem idealen Verfahren zur objektiven Beurteilung der Schwere von FD, dem MIDI-gestützten Verfahren zur Tonleiter-Analyse nach Jabusch (Jabusch et al. 2004b), hat ADDS eine gute Validität (Spector und Brandfonbrener 2007), d.h. eine gute Korrelation der Beurteilung der FD durch zwei verschiedene Methoden (z.B.: MIDI-gestützte Analyse und ADDS). Außerdem kann man mit der ADDS auch gut Veränderungen der Spielfähigkeit nach Interventionen (Responsiveness to Change) beurteilen (Spector und Brandfonbrener 2007). Die übrigen Kriterien haben wir ähnlich der ADDS gestaffelt. Dies bedeutete bei Rhythmus bzw. Gleichmäßigkeit des Anschlags: $0=$ keine Abweichung vom Metronom, 1= leichte Abweichung, 2= moderate Abweichung und 3= deutliche Abweichung vom Metronom. Bei der Regelmäßigkeit der Lautstärke: 0= keine Abweichung, 1= leichte Abweichung, 2= moderate Abweichung, $3=$ deutliche Abweichung. Bei Kriterium Klang: $0=$ nicht beeinträchtigt, $1=$ leicht beeinträchtigt, $2=$ moderat beeinträchtigt und $3=$ deutlich beeinträchtigt. Für die grobmotorischen Bewegungen galt: $0=$ keine abnormalen 
Bewegungen, 1= leichte abnormale Bewegungen, 2= moderate abnormale Bewegungen, 3= deutlich abnormale Bewegungen. Abschließend galt für den Gesamteindruck des Takes: 0= normales Spiel, 1= leichte Schwierigkeiten, 2= moderate Schwierigkeiten und 3= deutliche Schwierigkeiten. Zur besseren objektiven Quantifizierung der dystonen Bewegungen nahmen wir eine von Spector und Brandfonbrener entwickelte Methode, der Frequency of Abnormal Movements (FAM) (Spector und Brandfonbrener 2005), auf: Hierbei wird sowohl die Anzahl der dystonen, als auch der kompensatorischen Bewegungen gezählt. Wir haben die dystone und die kompensatorische Bewegung genau definiert und besonderen Wert darauf gelegt, dass die Rater diese gut verstanden haben, damit sie die Bewegungen möglichst einheitlich bewerten würden.

Demnach lautete die Definition für eine dystone Bewegung: der dystone Finger rollt sich nach dem Anschlag der Saite in eine abnormale Beugung ein und wird nicht in eine Ausgangsposition zurückgeführt, von welcher aus der Finger wieder spielbereit wäre.

Für die kompensatorische Bewegung galt: Streckung des kompensatorischen Fingers. Zwischen kompensatorischem Finger und mindestens einem anderen Finger entsteht ein Winkel von mindestens $30^{\circ}$ (hierbei gilt die Linie zwischen dem metacarpophalangealen Gelenk und dem proximalen Interphalangealgelenk der jeweiligen Finger, s. Abbildung 7).

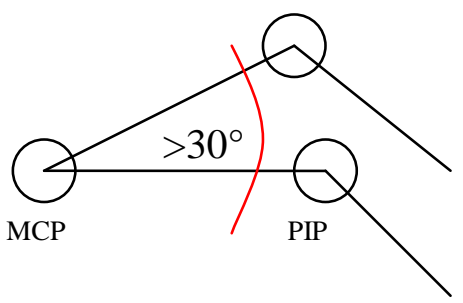

Abbildung 7 Fingerstellung bei kompensatorischer Bewegung, $\mathrm{MCP}=$ metakarpophalangeales Gelenk, $\mathrm{PIP}=$ proximales Interphalangealgelenk

Wenn mehrere Finger gleichzeitig kompensatorisch strecken, wird dies trotzdem als nur eine kompensatorische Bewegung gezählt. Bei permanenter kompensatorischer Streckung eines Fingers kann eine weitere kompensatorische Bewegung erst gezählt werden, wenn der Finger aus der Streckung in Neutralposition gegangen ist.

$\mathrm{Zu}$ jedem Probanden wurde den Ratern der oder die dystone(n) Finger genannt. Zum einfacheren Zählen der mitunter sehr schnell aufeinander folgenden dystonen Bewegungen bekamen die Rater Handzähler für die Auswertung. Die Rater wurden bei einem gemeinsamen Treffen genau instruiert, sie bekamen alle dieselben Informationen. Es wurde ihnen zum besseren Vergleich zu Beginn ein Take mit allen Übungen von einer gesunden Gitarristin demonstriert, den wir vorab auch auf Video aufgenommen hatten. Durch ein 
Proberating an einzelnen Takes wollten wir uns vorab über eine ausreichende Reliabilität vergewissern. D.h. eine gute Korrelation der Beurteilung der FD von einem Rater zwischen zwei verschiedenen Zeitpunkten (Intra-Rater Correlation), bzw. von einem Zeitpunkt zwischen zwei verschiedenen Ratern (Inter-Rater Correlation). Jeder Rater bekam zehn DVDs, worauf jeweils ein Proband mit seinen beiden Terminen enthalten war. Die Probanden waren anonymisiert und liefen nur unter einer Nummer (1-10). Auf jeder DVD waren 6 Takes (A-F). Zum 1. Termin gehörten Take A-C, zum 2. Termin Take D-F. Die Reihenfolge der Takes (vor, nach, 60 min nach Stimulation) wurde randomisiert. D.h., dass die Rater zwar wussten, zu welchem Termin ein Take gehört, ihn aber keinem Zeitpunkt (vor, unmittelbar nach, 60 min nach Stimulation) zuordnen konnten. Die Rater waren also geblindet.

\subsection{Reisekosten/Aufwandsentschädigung}

Sofern die Probanden ausschließlich für den Versuch angereist sind und nicht zusätzlich auch einen regulären Termin in der Musiker-Ambulanz bei Prof. Altenmüller hatten, wurden die Fahrtkosten erstattet. Jeder Proband erhielt für die Teilnahme eine Aufwandsentschädigung von 100 Euro.

\subsection{Sicherheitsvorkehrungen}

Während der Versuche war immer ein Arzt in Bereitschaft. Für den Fall eines epileptischen Anfalls wurde Diazepam bereitgehalten.

\subsection{Statistische Auswertung}

Die 13 verschiedenen Übungen jedes Takes wurden unter allen Kriterien von den drei Ratern beurteilt. Anschließend bildeten wir einen Mittelwert aus allen drei Beurteilungen. Hierfür wurden vorher alle Kriterien mithilfe der Intraklassenkorrelation (Cronbachs alpha) auf eine entsprechende Interrater-Reliabilität hin beurteilt (s.u.). Für die weitere statistische Analyse verwendeten wir die Mittelwerte der Rater. Für die statistische Auswertung rechneten wir eine zweifaktorielle Varianzanalyse mit Messwiederholung. Erster Faktor war die Stimulationsbedingung Verum oder Placebo, dementsprechend 2-stufig. Der zweite Faktor war der Zeitpunkt vor, unmittelbar nach oder 60 Minuten nach Stimulation, dementsprechend 3-stufig. Die übrigen Zeitpunkte (während, 30 Minuten und 120 Minuten nach Stimulation) wurden für die weitere Auswertung aufgrund der Länge des zu beurteilenden Filmmaterials nicht berücksichtigt.

Das Signifikanzniveau p setzten wir auf 0,05 fest. Neben der ANOVA wurden einzelne Ergebnisse mithilfe eines gepaarten T-Testes überprüft. Die Datenanalyse wurde mit dem 
Statistikprogramm SPSS Version 16 gerechnet (SPSS, Chicago, Illinois). Für die gesonderte Auswertung des Arpeggios (Übung 1) wurde aus den o. g. Mittelwerten für die einzelnen Kriterien Rhythmus, Lautstärke, Klang, ADDS und grobmotorische Bewegungen ein die Kriterien zusammenfassender Mittelwert gebildet. Außerdem wurde die Anzahl der dystonen und kompensatorischen Bewegungen zu einem Mittelwert zusammengefasst. Mit diesen beiden Mittelwerten wurde wiederum eine o. g. Varianzanalyse gerechnet. 


\section{Ergebnisse}

\subsection{Effekte kathodaler tDCS über dem primär motorischen Kortex}

\subsubsection{Interrater-Reliabilität}

Um möglichst reliable Beurteilungen der Probanden zu erlangen, bildeten wir für alle Kriterien (mit einer Ausnahme: grobmotorische Bewegungen) einen Mittelwert aus allen drei Ratern. Voraussetzung hierfür ist jedoch eine hinreichende Übereinstimmung der individuellen Urteile der einzelnen Rater (Bortz und Döring 2006). Liegen, wie in unserem Fall, intervallskalierte Ratingwerte vor, stellt die Intraklassenkorrelation (ICC) die angemessene Methode zur Reliabilitätsbestimmung dar (Wirtz und Caspar 2002). Die justierte ICC des Mittelwerts mehrerer Rater (justiert = es wurden alle Probanden von allen Ratern beurteilt und die Interrater-Reliabilität ist begrenzt auf die drei tatsächlich an der Studie teilnehmenden Rater und ist nicht im Sinne einer Raterstichprobe übertragbar auf eine Population) als Maß für die Interrater-Raliabilität kann mittels Cronbachs- $\alpha$ berechnet werden (Wirtz und Caspar 2002). ICC-Werte > 0,7 gelten in der Literatur als ein Indiz für gute Reliabilität (Greve und Ventura 1997). Wir haben ICC-Werte für jedes Kriterium zu den verschiedenen Bedingungen (Zeitpunkt und Verum/Placebo) errechnet (s. Tabelle 2). Unser mittlerer ICC-Wert lag bei 0,87 $\pm 0,09$ (Standardabweichung). Der höchste ICC-Wert von 0,97 ergab sich bei Kriterium Gesamteindruck (vor Stimulation, tDCS), der niedrigste ICCWert von 0,56 ergab sich bei Kriterium Lautstärke (nach Stimulation, tDCS).

Tabelle 2 Intraklassenkorrelation für alle Kriterien

\begin{tabular}{|l|l|l|l|l|l|l|}
\hline \multirow{2}{*}{ ICC (Cronbachs alpha) } & \multicolumn{2}{|c|}{ Vor Stimulation } & \multicolumn{2}{c|}{ Nach Stimulation } & \multicolumn{2}{c|}{ 60 min nach Stimulation } \\
\cline { 2 - 7 } & tDCS & Placebo & tDCS & Placebo & tDCS & Placebo \\
\hline Gesamteindruck & 0,97 & 0,76 & 0,76 & 0,90 & 0,90 & 0,81 \\
\hline Rhythmus & 0,94 & 0,94 & 0,74 & 0,89 & 0,92 & 0,96 \\
\hline Lautstärke & 0,86 & 0,79 & 0,56 & 0,92 & 0,88 & 0,79 \\
\hline Klang & 0,86 & 0,93 & 0,74 & 0,78 & 0,87 & 0,85 \\
\hline ADDS & 0,91 & 0,87 & 0,85 & 0,86 & 0,91 & 0,92 \\
\hline grobmotorische Bewegungen & 0,96 & 0,96 & 0,93 & 0,96 & 0,97 & 0,97 \\
\hline dystone Bewegungen & 0,90 & 0,88 & 0,95 & 0,71 & 0,94 & 0,93 \\
\hline $\begin{array}{l}\text { kompensatorische } \\
\text { Bewegungen }\end{array}$ & 0,88 & 0,88 & 0,81 & 0,82 & 0,67 & 0,90 \\
\hline
\end{tabular}

Die Intraklassenkorrelation (ICC) wurde mittels Cronbachs- $\alpha$ bestimmt

Werte $>\mathbf{0 , 7}$ gelten als gut

\subsubsection{Gesamteindruck}

Um in die Analyse der Ergebnisse einzusteigen, bietet sich der Gesamteindruck an, der von jedem Rater für jeden Take beurteilt werden musste. Hier zeigt sich, dass insgesamt keine 
deutlichen Spielverbesserungen unter Stimulationsbedingungen resultierten. Beurteilt wurden die Zeitpunkte vor (V), unmittelbar nach (N) und 60 Minuten nach Stimulation (S). Die Probanden kamen zu zwei Sitzungen, einer Verum-Stimulation (R, wie Real) und einer Placebo-Stimulation (P, wie Placebo). Daraus ergeben sich für die einzelnen Zeitpunkte die Abkürzungen VR, NR, SR, VP, NP und SP. Eine zweifaktorielle Varianzanalyse (ANOVA) mit Messwiederholungen (2-stufige Bedingung: Verum und Placebo sowie 3-stufiger Zeitpunkt: vor, unmittelbar nach und $60 \mathrm{~min}$ nach Stimulation) für das Kriterium Gesamteindruck ergab ebenfalls keine signifikanten Unterschiede, weder für die Bedingung $\left(\mathrm{F}(1,10)=0,03 ; \mathrm{p}=0,86 ; \eta^{2}=0,004\right)$ noch für den Zeitpunkt $\left(\mathrm{F}(2,10)=0,27 ; \mathrm{p}=0,77 ; \eta^{2}=\right.$ 0,03). Auch die Interaktion Bedingung $\times$ Zeit ergibt keine Signifikanz $\left(F=0,06 ; p=0,95,{ }^{2}\right.$ $=0,006)$.

Als einziger wurde Proband 1 im Verlauf besser bewertet. Hierauf wird in einem späteren Teil (einzelne Probanden) näher eingegangen.

Tabelle 3 statistische Auswertung Gesamteindruck und Selbsteinschätzung, gerechnet wurde eine zweifaktorielle ANOVA mit Messwiederholung, $\mathrm{P}=$ Signifikanzniveau

\begin{tabular}{|l|l|l|l|l|l|l|l|l|l|}
\hline ANOVA alle Probanden & \multicolumn{2}{l}{ Faktor 1: Bedingung } & \multicolumn{2}{l|}{ Faktor 2: Zeit } & \multicolumn{2}{l|}{ Interaktion: Bedingung $\times$ Zeit } \\
\cline { 2 - 11 } & $\mathbf{P}$ & $\mathbf{F}$ & $\mathbf{\eta}^{\mathbf{2}}$ & $\mathbf{P}$ & $\mathbf{F}$ & $\mathbf{\eta}^{2}$ & $\mathbf{P}$ & $\mathbf{F}$ & $\boldsymbol{\eta}^{2}$ \\
\hline Gesamteindruck & 0,86 & 0,03 & 0,004 & 0,77 & 0,27 & 0,03 & 0,95 & 0,06 & 0,006 \\
\hline Selbsteinschätzung & 0,97 & 0,002 & 0,0 & 0,91 & 0,09 & 0,01 & 0,94 & 0,06 & 0,006 \\
\hline
\end{tabular}

Auch in der Selbsteinschätzung, die jeder Proband angeben musste, lassen sich keine deutlichen Verbesserungen ausmachen. Eine ANOVA ist weder für Faktor Bedingung $\left(F(1,10)=0,002 ; p=0,97 ; \eta^{2}=0,0\right)$, noch für Faktor Zeitpunkt $\left(F(2,10)=0,09 ; p=0,91 ; \eta^{2}=\right.$ $0,01)$ signifikant. Auch die Interaktion Bedingung $\times$ Zeit ist nicht signifikant $(F=0,06 ; p=$ 0,94; $\left.\eta^{2}=0,006\right)$. Allerdings gibt Proband 1 passend zum objektiven Gesamteindruck auch eine subjektive Verbesserung der Spielfähigkeit um $10 \%$ an.

Abbildung 3.1.2-1 Gesamteindruck unter Verum-Bedingung;

Abbildung 3.1.2-2 Gesamteindruck unter PlaceboBedingung;

VR, NR und SR= Zeitpunkte

VP, NP und SP= Zeitpunkte
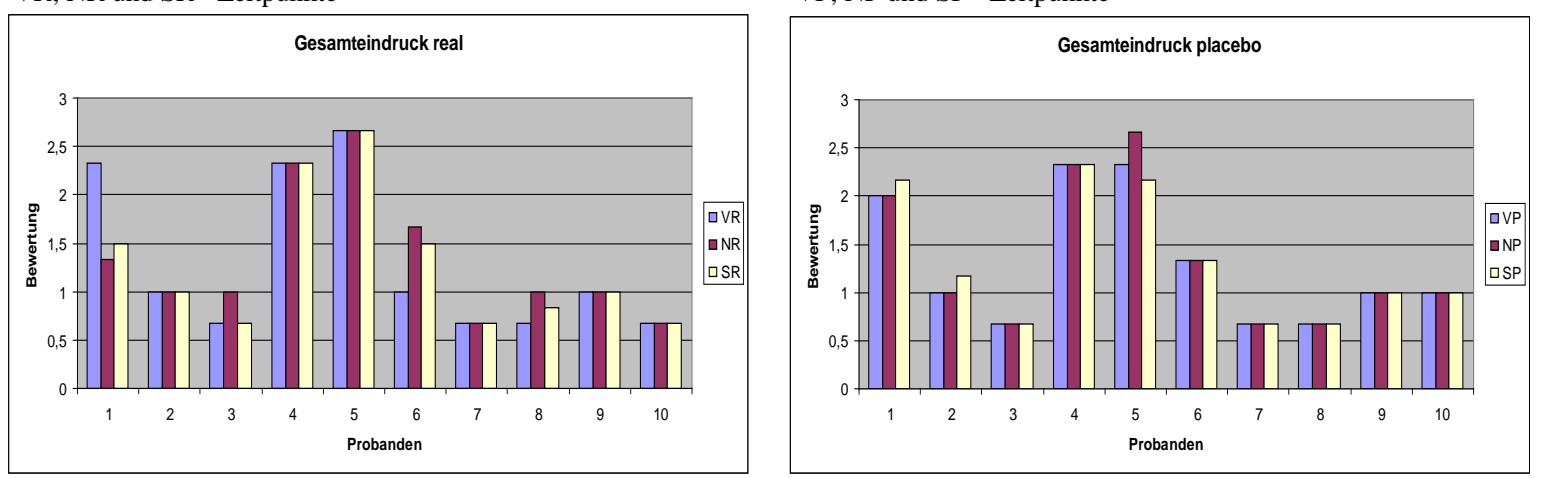

für beide Diagramme gilt: 0= normales Spiel, 1= leichte Schwierigkeiten, 2= moderate Schwierigkeiten und 3= deutliche Schwierigkeiten 
Abbildung 3.1.2-3 Selbsteinschätzung unter VerumBedingung; VR, NR und SR= Zeitpunkte; $100 \%=$ unbeeinträchtigte Spielfähigkeit

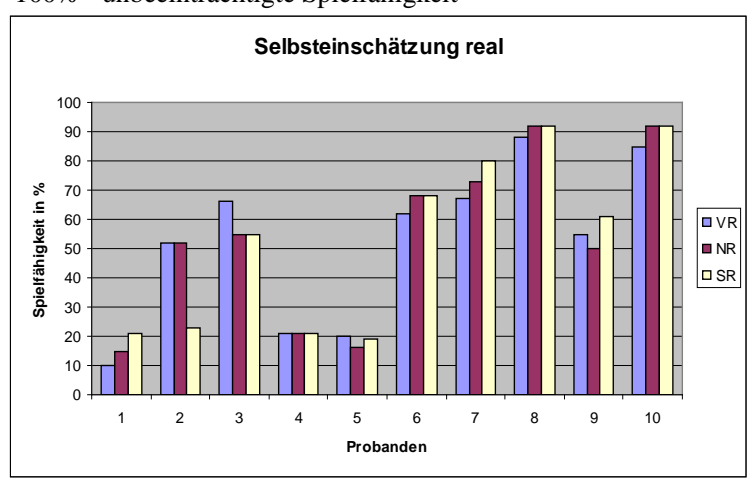

Abbildung 3.1.2-4 Selbsteinschätzung unter PlaceboBedingung; VP, NP und SP= Zeitpunkte; $100 \%=$ unbeeinträchtigte Spielfähigkeit

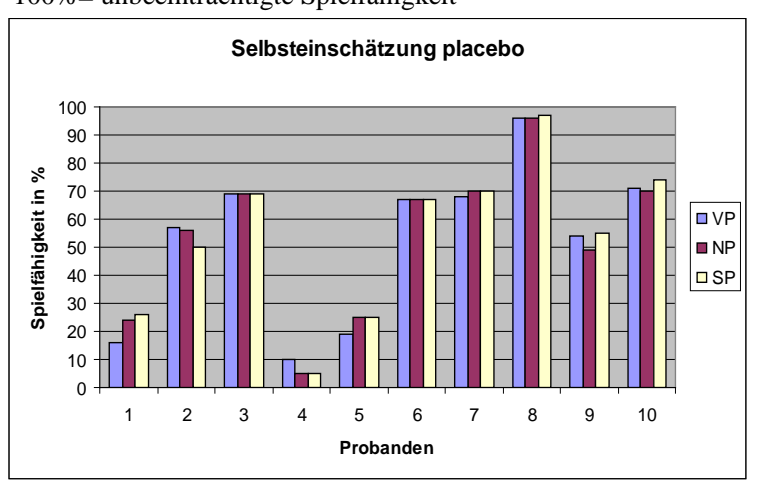

\subsubsection{Einzelne Kriterien}

Unter dem Kriterium Rhythmus zeigte sich unter Verum-Stimulation keine deutliche Verbesserung. Lediglich die Probanden 5 und 8 zeigen eine leichte Verbesserung, die sich aber auch unter Placebo-Bedingung zeigt. Eine zweifaktorielle ANOVA mit Messwiederholung ergibt keine signifikanten Veränderungen (Bedingung: $F(1,10)=1,19$; $p$ $=0,30 ; \eta^{2}=0,12$; Zeitpunkt: $F(2,10)=0,41 ; p=0,67 ; \eta^{2}=0,04$; Interaktion Bedingung $\times$ Zeit: $\left.F=0,31 ; p=0,74 ; \eta^{2}=0,03\right)$.

Das Kriterium Lautstärke zeigt eine leichte Verbesserung der Gleichmäßigkeit bei Proband 1, daneben verbessert sich Proband 4 unmittelbar nach Stimulation um eine Einheit, was sich aber zum Zeitpunkt 60 min nach Stimulation wieder relativiert. Bei den übrigen Probanden sind keine deutlichen Verbesserungen im Verlauf sichtbar. Eine zweifaktorielle ANOVA mit Messwiederholung ergibt keine signifikanten Ergebnisse (Bedingung: $F(1,10)=0,02 ; p=$ 0,$90 ; \eta^{2}=0,002$; Zeitpunkt: $\mathrm{F}(2,10)=0,49 ; \mathrm{p}=0,62 ; \eta^{2}=0,05$; Interaktion Bedingung $\times$ Zeit: $\left.\mathrm{F}=1,98 ; \mathrm{p}=0,17 ; \eta^{2}=0,18\right)$.

Tabelle 4 statistische Auswertung aller Kriterien, gerechnet wurde eine zweifaktorielle ANOVA mit Messwiederholung, $\mathrm{P}=$ Signifikanzniveau

\begin{tabular}{|l|l|l|l|l|l|l|l|l|l|l|}
\hline \multirow{2}{*}{ ANOVA alle Probanden } & \multicolumn{3}{|l}{ Faktor 1: Bedingung } & \multicolumn{3}{l|}{ Faktor 2: Zeit } & \multicolumn{3}{|l|}{ Interaktion: Bedingung Zeit } \\
\cline { 2 - 11 } & $\mathbf{P}$ & $\mathbf{F}$ & $\mathbf{\eta}^{\mathbf{2}}$ & $\mathbf{P}$ & $\mathbf{F}$ & $\mathbf{\eta}^{2}$ & $\mathbf{P}$ & $\mathbf{F}$ & $\boldsymbol{\eta}^{2}$ \\
\hline Gesamteindruck & 0,86 & 0,03 & 0,004 & 0,77 & 0,27 & 0,03 & 0,95 & 0,06 & 0,006 \\
\hline Selbsteinschätzung & 0,97 & 0,002 & 0,0 & 0,91 & 0,09 & 0,01 & 0,94 & 0,06 & 0,006 \\
\hline Rhythmus & 0,30 & 1,19 & 0,12 & 0,67 & 0,41 & 0,04 & 0,74 & 0,31 & 0,03 \\
\hline Lautstärke & 0,90 & 0,02 & 0,002 & 0,62 & 0,49 & 0,05 & 0,17 & 1,98 & 0,18 \\
\hline Klang & 0,77 & 0,09 & 0,01 & 0,28 & 1,36 & 0,13 & 0,03 & 4,26 & 0,32 \\
\hline ADDS & 0,92 & 0,01 & 0,001 & 0,32 & 1,21 & 0,12 & 0,86 & 0,15 & 0,02 \\
\hline $\begin{array}{l}\text { grobmotorische } \\
\text { Bewegungen (2 Rater) }\end{array}$ & 0,18 & 2,23 & 0,24 & 0,58 & 0,57 & 0,08 & 0,22 & 1,69 & 0,20 \\
\hline dystone Bewegungen & 0,81 & 0,06 & 0,007 & 0,09 & 2,83 & 0,24 & 0,67 & 0,42 & 0,04 \\
\hline $\begin{array}{l}\text { kompensatorische } \\
\text { Bewegungen }\end{array}$ & 0,06 & 4,46 & 0,33 & 0,22 & 1,67 & 0,16 & 0,64 & 0,47 & 0,05 \\
\hline
\end{tabular}


Abbildung 3.1.3-1 Kriterium Rhythmus unter VerumBedingung; VR, NR und SR= Zeitpunkte

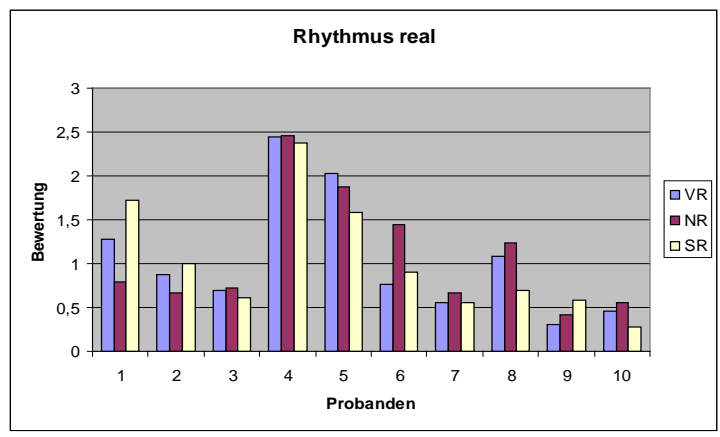

Abbildung 3.1.3-2 Kriterium Rhythmus unter PlaceboBedingung; VP, NP und SP= Zeitpunkte

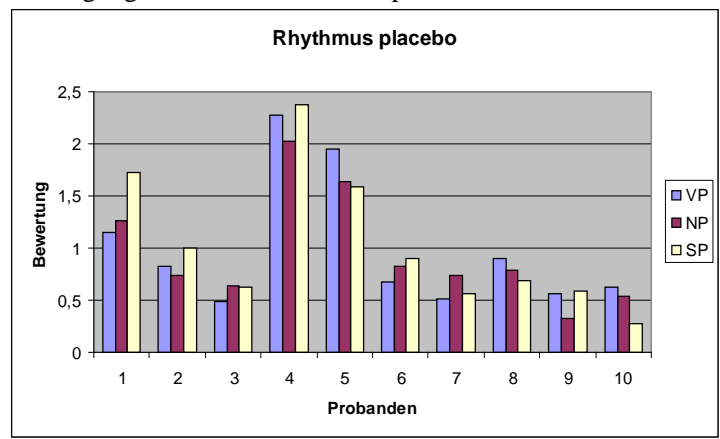

für beide Diagramme gilt: $0=$ keine Abweichung vom Metronom, 1= leichte Abweichung, 2= moderate Abweichung und 3= deutliche Abweichung vom Metronom

Abbildung 3.1.3-3 Kriterium Gleichmäßigkeit der Lautstärke unter Verum-Bedingung; VR, NR und SR= Zeitpunkte

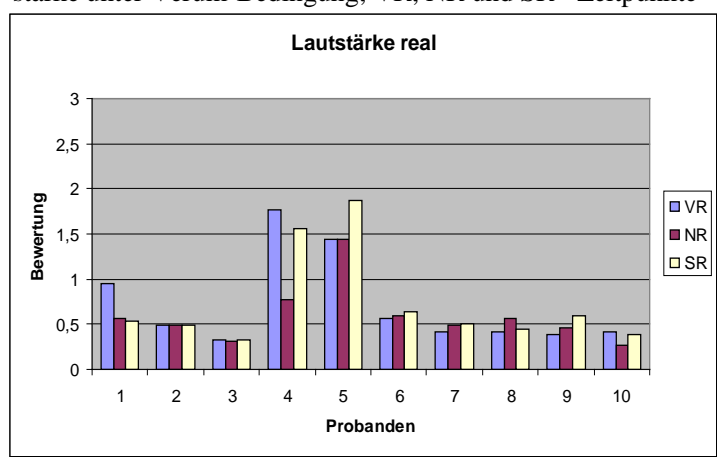

Abbildung 3.1.3-4 Kriterium Gleichmäßigkeit der Lautstärke unter Placebo-Bedingung; VP, NP und SP= Zeitpunkte

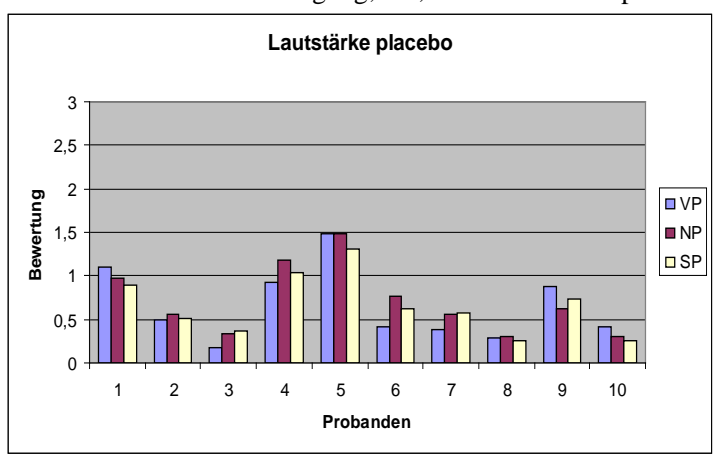

für beide Diagramme gilt: $0=$ keine Abweichung, $1=$ leichte Abweichung, $2=$ moderate Abweichung, $3=$ deutliche Abweichung

Das Kriterium Klang zeigt ebenfalls eine leichte Verbesserung bei Proband 1, außerdem zeigt sich bei den Probanden 4 und 5 eine Verbesserung des Klangs unmittelbar nach Stimulation von 0,6 und 0,3 Einheiten. Diese Verbesserung kehrt sich allerdings zum Zeitpunkt 60 min nach Stimulation wieder um. Ansonsten gibt es keine bemerkenswerten Veränderungen. Eine zweifaktorielle ANOVA mit Messwiederholung ergibt keine signifikanten Ergebnisse für die Faktoren Bedingung und Zeitpunkt (Bedingung: $\mathrm{F}(1,10)=0,09 ; \mathrm{p}=0,77 ; \eta^{2}=0,01$; Zeitpunkt: $\left.F(2,10)=1,36 ; p=0,28 ; \eta^{2}=0,13\right)$. In der Interaktion Bedingung $\times$ Zeit allerdings ergibt sich eine Signifikanz $\left(F=4,26 ; p=0,03 ; \eta^{2}=0,32\right)$. Eine statistische Überprüfung mithilfe eines gepaarten T-Testes, kann diese Signifikanz aber nicht bestätigen (s. Tabelle 5). 
Abbildung 3.1.3-5 Kriterium Klang unter VerumBedingung; VR, NR und SR= Zeitpunkte

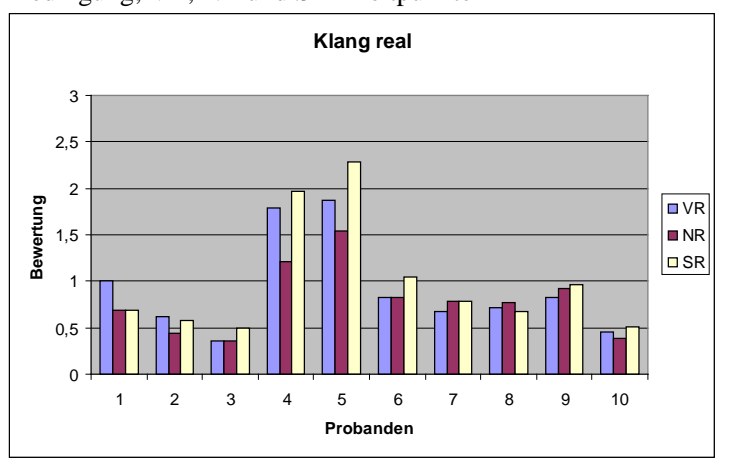

Abbildung 3.1.3-6 Kriterium Klang unter PlaceboBedingung; VP, NP und SP= Zeitpunkte

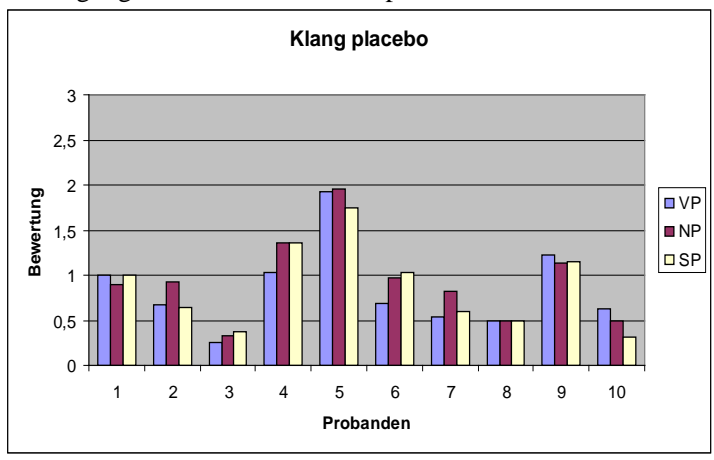

für beide Diagramme gilt: $0=$ nicht beeinträchtigt, $1=$ leicht beeinträchtigt, $2=$ moderat beeinträchtigt, $3=$ deutlich beeinträchtigt

Tabelle 5 statistische Überprüfung des Kriteriums Klang mithilfe eines T-Tests, die Mittelwerte der einzelnen Zeitpunkte aller Probanden wurden miteinander verglichen

\begin{tabular}{|l|l|l|l|l|l|l|l|l|l|}
\hline T-Test Klang & VP-VR & NP-NR & SP-SR & NR-VR & SR-VR & SR-NR & NP-VP & SP-VP & SP-NP \\
\hline Signifikanz (2-seitig) & 0,50 & 0,07 & 0,18 & 0,13 & 0,17 & 0,06 & 0,15 & 0,72 & 0,17 \\
\hline
\end{tabular}

Abbildung 3.1.3-7 Mittelwert aller Probanden für das Kriterium Klang; Linie am Ende der Balken zeigt den Standardfehler

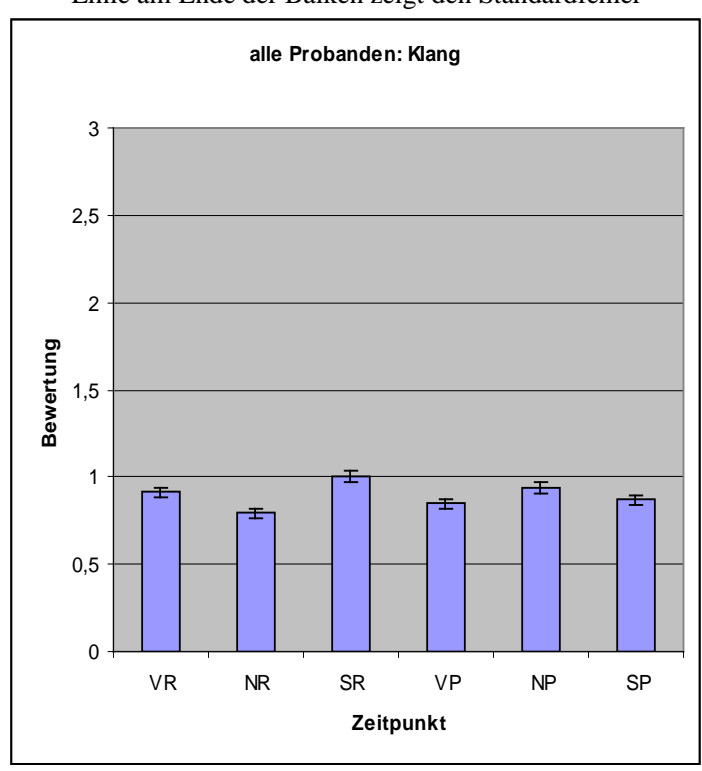


Unter Kriterium ADDS ergeben sich wie in den bisherigen Kriterien keine deutlichen Veränderungen, außer einer leichten Verbesserung bei Proband 1. Eine zweifaktorielle ANOVA mit Messwiederholung ergibt keine signifikanten Ergebnisse (Bedingung: $F(1,10)=$ 0,$01 ; \mathrm{p}=0,92 ; \eta^{2}=0,001 ;$ Zeitpunkt: $\mathrm{F}(2,10)=1,21 ; \mathrm{p}=0,32 ; \eta^{2}=0,12$; Interaktion Bedingung $\times$ Zeit: $\left.F=0,15 ; \mathrm{p}=0,86 ; \eta^{2}=0,02\right)$.
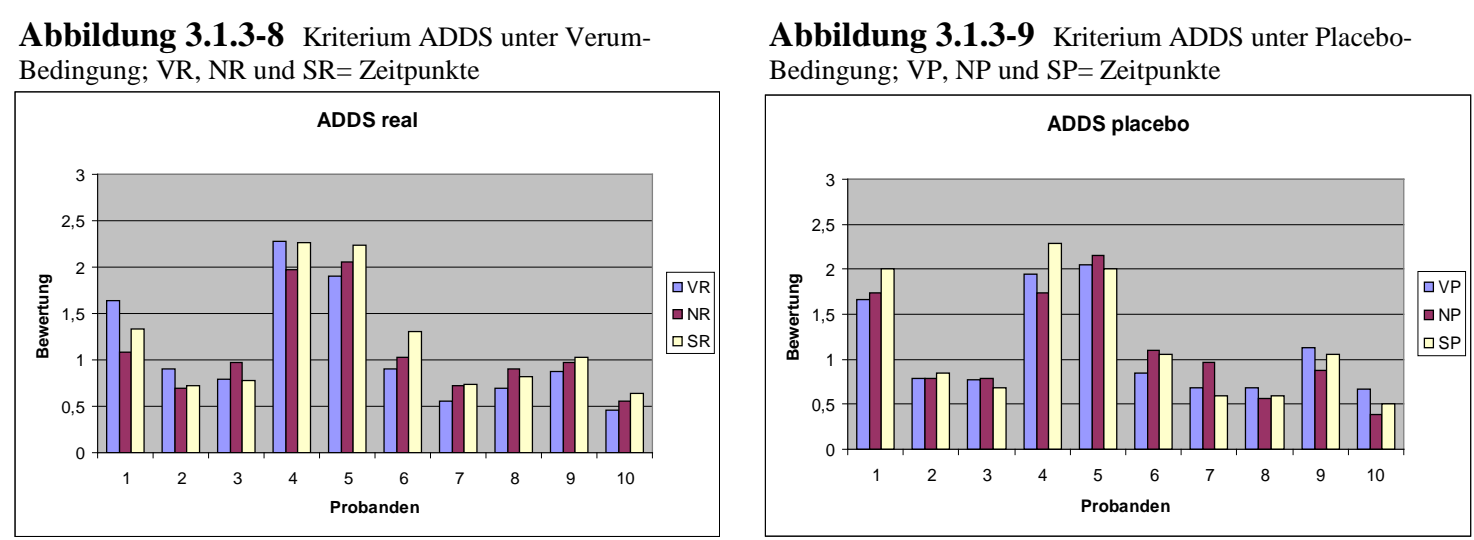

für beide Diagramme gilt: $0=$ normales Spiel, $1=$ leichte Schwierigkeiten, $2=$ moderate Schwierigkeiten, $3=$ deutliche Schwierigkeiten

Für die Kriterien dystone und kompensatorische Bewegungen wurde die Anzahl der unphysiologischen Bewegungen für jede einzelne Übung ausgezählt. Da viele Übungen nur sehr kurz waren und hier von den meisten Probanden keine unphysiologischen Bewegungen gemacht wurden, scheint es sinnvoll, für diese Kriterien nur die erste und umfangreichste Übung, das Arpeggio, zu berücksichtigen. Diese Übung ging meist über 1-2 Minuten und ist für die Anzahl der unphysiologischen Bewegungen am repräsentativsten. Die Diagramme zeigen, dass es zwar teilweise deutliche Veränderungen innerhalb der einzelnen Zeitpunkte gab (Proband 2 und 3), allerdings keine Verbesserungen. Für die dystonen Bewegungen ergab eine zweifaktorielle ANOVA mit Messwiederholung keine signifikanten Veränderungen (Bedingung: $F(1,10)=0,01 ; p=0,94 ; \eta^{2}=0,001 ;$ Zeitpunkt: $F(2,10)=1,14 ; p=0,34 ; \eta^{2}=$ 0,11; Interaktion Bedingung $\times$ Zeit: $\left.F=0,42 ; p=0,67 ; \eta^{2}=0,04\right)$. Für die kompensatorischen Bewegungen ergaben sich ebenfalls keine signifikanten Ergebnisse (Bedingung: $F(1,10)=$ 0,$16 ; \mathrm{p}=0,70 ; \eta^{2}=0,02 ;$ Zeitpunkt: $\mathrm{F}(2,10)=1,33 ; \mathrm{p}=0,29 ; \eta^{2}=0,13$; Interaktion Bedingung $\times$ Zeit: $\left.F=0,47 ; p=0,64 ; \eta^{2}=0,05\right)$. 
Abbildung 3.1.3-10 Anzahl dystoner Bewegungen während des Arpeggios unter Verum-Bedingung; VR, NR und SR= Zeitpunkte

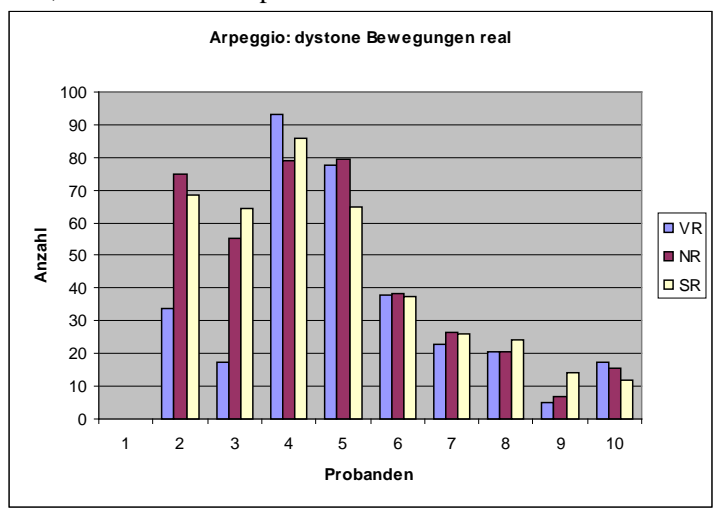

Abbildung 3.1.3-12 Anzahl kompensatorischer Bewegungen während des Arpeggios unter Verum-Bedingung; VR, NR und SR= Zeitpunkte

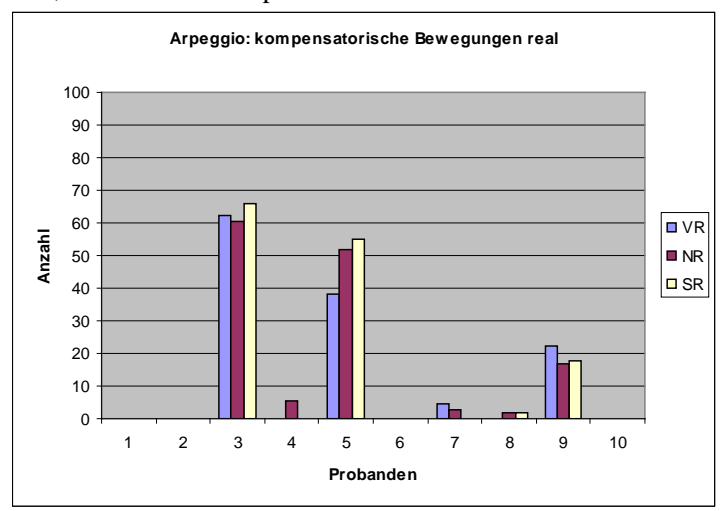

Abbildung 3.1.3-11 Anzahl dystoner Bewegungen während des Arpeggios unter Placebo-Bedingung. VP, NP und SP= Zeitpunkte

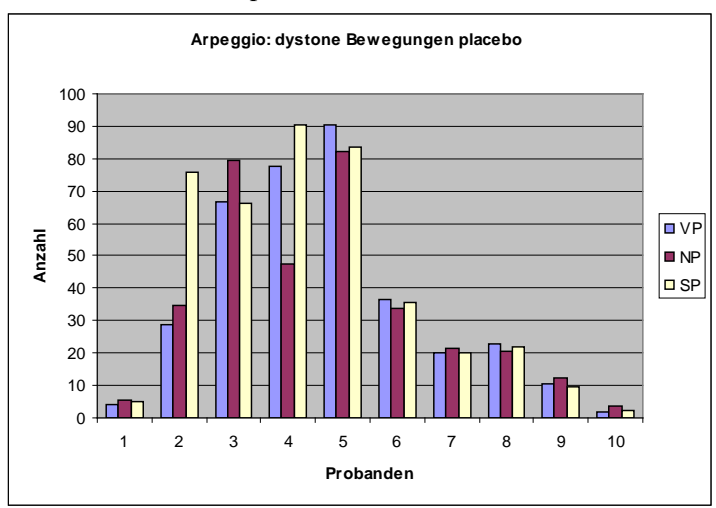

Abbildung 3.1.3-13 Anzahl kompensatorischer Bewegungen während des Arpeggios unter Placebo-Bedingung; VP, NP und SP $=$ Zeitpunkte

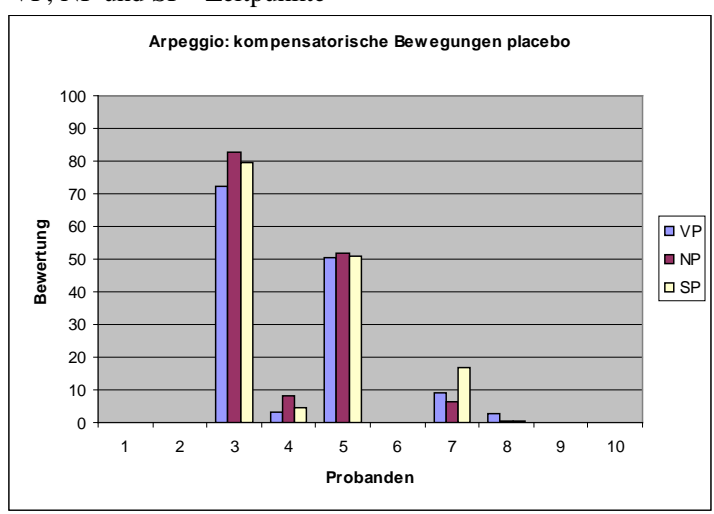

Auch unter dem Aspekt grobmotorische Bewegungen zeigten sich keine deutlichen Veränderungen (s. Abbildungen 3.1.3-13 und 3.1.3-14), ausgenommen Proband 1, bei dem wieder eine leichte Verbesserung unter Verum-Stimulation zu sehen ist. Eine zweifaktorielle ANOVA mit Messwiederholung ergab auch hier keine signifikanten Ergebnisse (Bedingung: $\mathrm{F}(1,10)=2,23 ; \mathrm{p}=0,18 ; \eta^{2}=0,24 ;$ Zeitpunkt: $\mathrm{F}(2,10)=0,57 ; \mathrm{p}=0,58 ; \eta^{2}=0,08$; Interaktion Bedingung $\times$ Zeit: $F=1,69 ; \mathrm{p}=0,22 ; \eta^{2}=0,20$ ).

Abbildung 3.1.3-14 Kriterium grobmotorische Bewegungen unter Verum-Bedingung; VR, NR und SR= Zeitpunkte

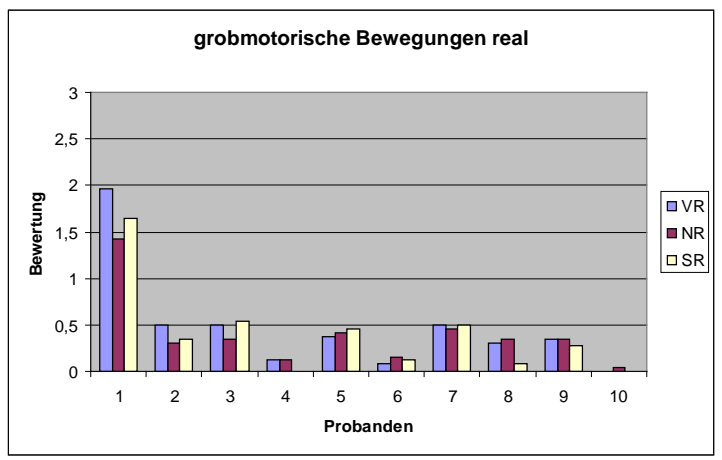

Abbildung 3.1.3-15 Kriterium grobmotorische Bewegungen unter Placebo-Bedingung; VP, NP und SP= Zeitpunkte

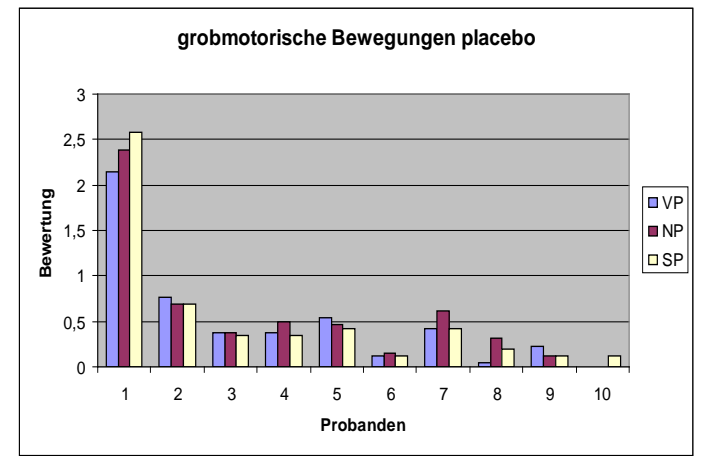

für beide Diagramme gilt: $0=$ keine abnormalen Bewegungen, $1=$ leichte abnormale Bewegungen, $2=$ moderate abnormale Bewegungen, $3=$ deutlich abnormale Bewegungen 


\subsubsection{Einzelne Probanden (Proband 1)}

Als einziger der zehn Probanden zeigte Proband 1 im Verlauf eine Verbesserung der Spielfähigkeit unter Verum-Stimulation (s Abbildungen 3.1.4-1 und 3.1.4-2). Betrachtet man auch hier zuerst den Gesamteindruck, ergibt sich eine objektive Verbesserung der Spielfähigkeit von 34\% unter Verum-Bedingung (VR 22\% - SR 56\%). Unter PlaceboBedingung verschlechtert sich die Spielfähigkeit um 6\% (VP 33\% - SP 28\%). Subjektiv schätzte sich der Proband bei beiden Terminen im Verlauf jeweils besser ein (VR 10\% - SR 21\%, VP 16\% - SP 26\%). Unter Kriterium ADDS verbesserte sich das Spiel bei VerumStimulation um 11\% (VR 45\% - SR 56\%), bei Placebo-Stimulation verschlechterte es sich um 12\% (VP 45\% - SP 33\%). Die Gleichmäßigkeit des Rhythmus verschlechterte sich sowohl unter Verum-, als auch unter Placebo-Bedingung (VR 57\% - SR 43\%, VP 62\% - SP 43\%). Die Regelmäßigkeit der Lautstärke verbesserte sich unter Verum-Bedingung um 14\% (VR 68\% -SR 82\%), unter Placebo-Bedingung um 7\% (VP 63\% - SP 70\%). Das Kriterium Klang verbesserte sich unter Verum-Stimulation um 10\% (VR 67\% - SR 77\%), unter PlaceboStimulation blieb es gleich (VP 67\% - SP 67\%).

Wegen einer etwas anders ausgeprägten Form der fokalen Dystonie (s. Teil 2.1 Probanden und 4.2 Verbesserung der Spielfähigkeit bei Proband 1), liegt ein besonderes Augenmerk bei Proband 1 nicht auf den dystonen oder kompensatorischen, sondern auf den grobmotorischen Bewegungen. Diese verbesserten sich unter Verum-Stimulation um 10\% (VR 35\% - SR 45\%), wohingegen sie sich unter Placebo-Stimulation um 14\% verschlechterten (VP 28\% -SP $14 \%)$. 
Abbildung 3.1.4-1 Proband 1 alle Kriterien unter Verum-Bedingung

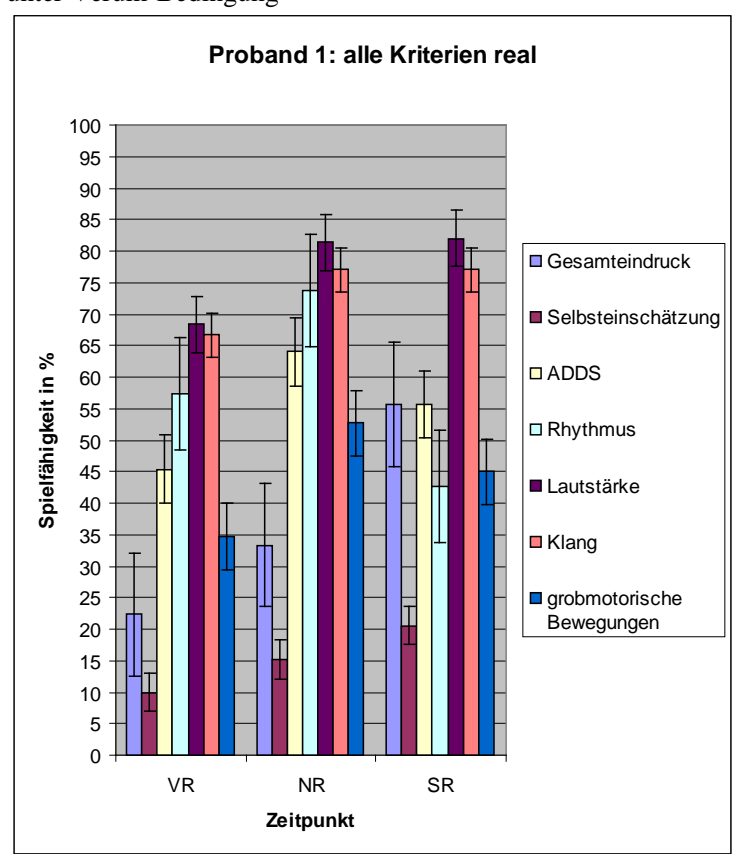

Abbildung 3.1.4-2 Proband 1 alle Kriterien unter Placebo-Bedingung

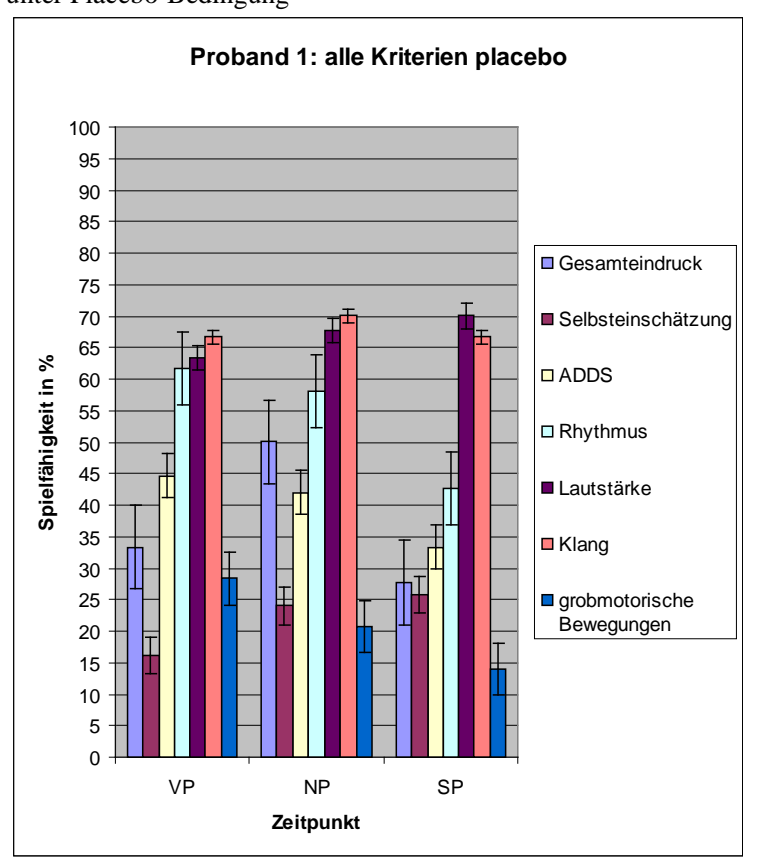

Für die Kriterien Gesamteindruck, ADDS, Rhythmus, Lautstärke, Klang \& grobmotorische Bewegungen erfolgte die Umrechnung der Bewertungsskala 0-3 ( $0=$ keine - 3=deutliche Beeinträchtigung) in eine prozentuale Angabe der Spielfähigkeit anhand der Formel: $\mathrm{y}=(3-\mathrm{x}) * 33,34 ; 100 \%$ bedeutet normales Spielvermögen, ohne jegliche Beeinträchtigung; Linie am Ende der Balken zeigt den Standardfehler

\subsubsection{Gruppierte Probanden (Erkrankungsdauer der Dystonie)}

Ein Aspekt der Beeinflussbarkeit der Dystonie ist die Dauer der Erkrankung. Besteht die Dystonie erst kürzere Zeit, ist die Wahrscheinlichkeit, die falschen Bewegungsmuster zu korrigieren, größer (Jabusch und Altenmüller 2006). Bei fünf unserer Probanden bestand die Dystonie erst seit 2 Jahren, bzw. kürzer als 2 Jahre (Proband 4: 0,5 Jahre; Probanden 3 \& 10: 1 Jahr; Probanden 6 \& 8: 2 Jahre). Die übrigen fünf Probanden hatten schon seit mehr als 2 Jahren Symptome der Dystonie (Proband 1: 11 Jahre; Proband 2: 9 Jahre; Proband 5: 18 Jahre; Proband 7: 17 Jahre; Proband 9: 26 Jahre) Die Gegenüberstellung der beiden Gruppen zeigt auch unter diesem Aspekt keinen Unterschied des Effekts durch tDCS. Außer bei oben besprochenem Probanden 1 zeigt sich in keiner der beiden Gruppen unter keinem Kriterium eine deutliche Verbesserung.

Abbildung 3.1.5-1 Gesamteindruck Dystonie $\leq 2$ Jahre unter Verum-Bedingung; VR, NR und SR= Zeitpunkte

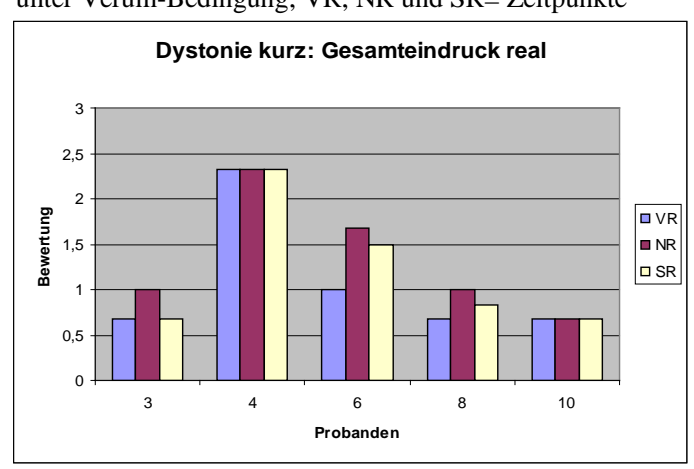

Abbildung 3.1.5-2 Gesamteindruck Dystonie $>2$ Jahre unter Verum-Bedingung; VR, NR und SR= Zeitpunkte

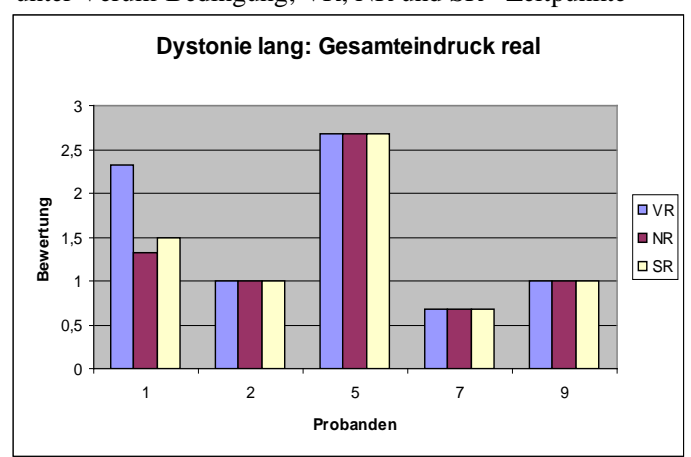


Abbildung 3.1.5-3 alle Kriterien bei Dystonie $\leq 2$ Jahre unter Verum-Bedingung

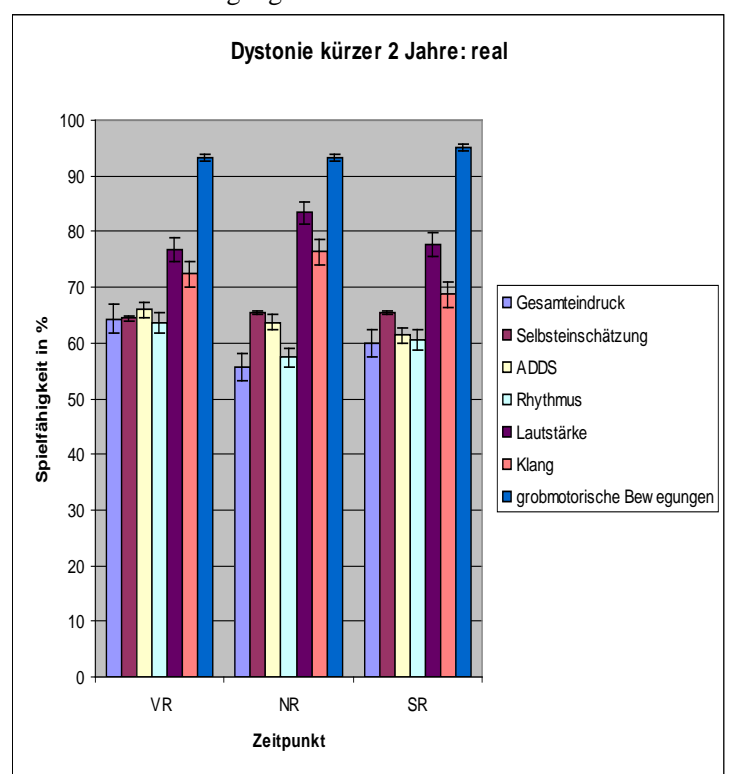

Abbildung 3.1.5-4 alle Kriterien bei Dystonie > 2 Jahre unter Verum-Bedingung

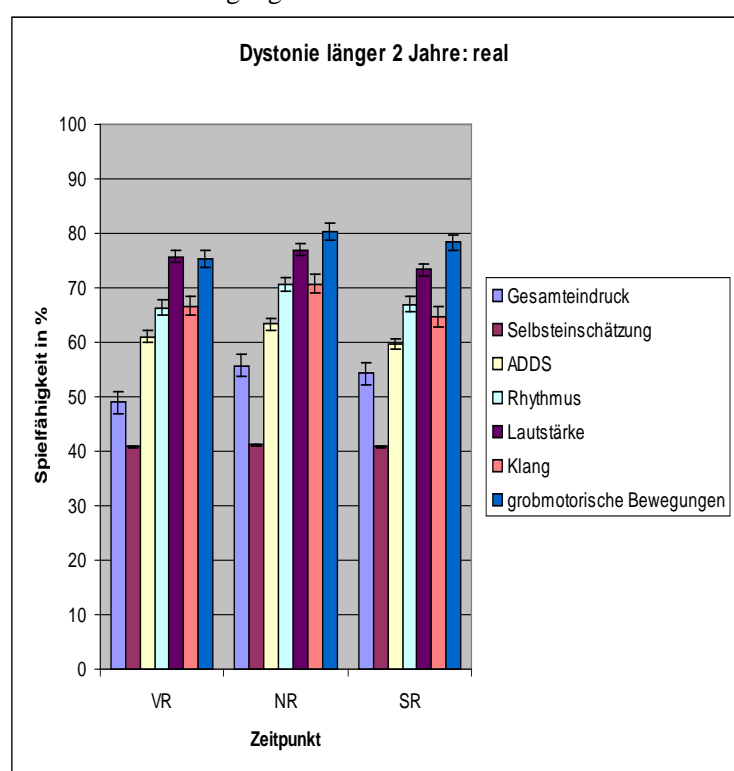

Für die Kriterien Gesamteindruck, ADDS, Rhythmus, Lautstärke, Klang \& grobmotorische Bewegungen erfolgte die Umrechnung der Bewertungsskala 0-3 ( $0=$ keine - 3=deutliche Beeinträchtigung) in eine prozentuale Angabe der Spielfähigkeit anhand der Formel: $\mathrm{y}=(3-\mathrm{x}) * 33,34 ; 100 \%$ bedeutet normales Spielvermögen, ohne jegliche Beeinträchtigung; Linie am Ende der Balken zeigt den Standardfehler

Abbildung 3.1.5-5 dystone und kompensatorische Bewegungen bei Dystonie $\leq 2$ Jahre unter Verum-Bedingung;

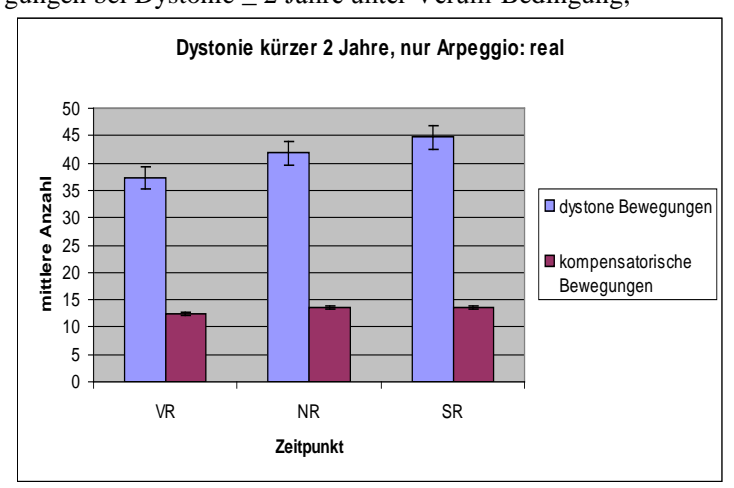

Abbildung 3.1.5-6 dystone und kompensatorische Bewegungen bei Dystonie > 2 Jahre unter Verum-Bedingung;

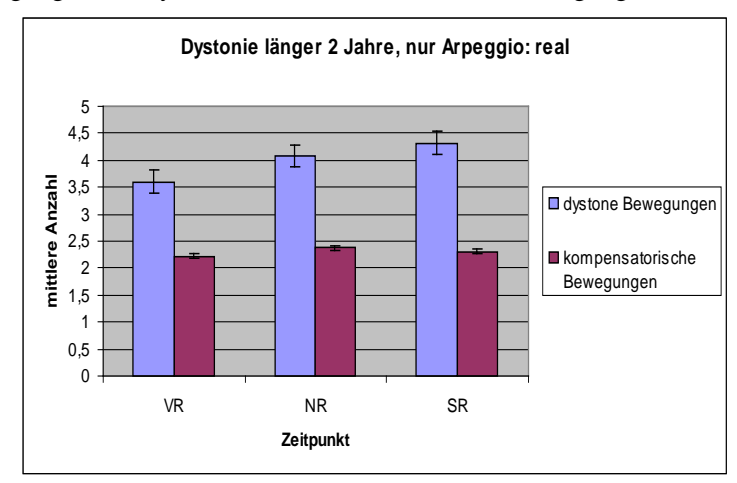

für beide Diagramme gilt: Linie am Ende der Balken zeigt den Standardfehler

\subsubsection{Einzelne Übungen (Arpeggio)}

Nachfolgend wird ein einzelne Übung, das Arpeggio, betrachtet. Es ist in meinen Augen sehr repräsentativ, weil es die einzige längere Übung über 1-2 Minuten war. Hier konnten auch die dystonen, bzw. kompensatorischen Bewegungen am besten ausgezählt werden. In den ersten Diagrammen sind alle Kriterien zu einem Mittelwert zusammengenommen, die mittels einer Likert-Scala (0-3) zu bewerten waren (Rhythmus, Lautstärke, Klang, ADDS \& grobmotorische Bewegungen). Es zeigt sich unter Verum-Bedingung wieder eine deutliche Verbesserung bei dem Probanden 1 um 0,7 Einheiten, leichte Verbesserungen bei Probanden 2, 3 und 7. Dieselben Probanden weisen unter Placebo-Bedingung keine Verbesserungen auf. 
Eine zweifaktorielle ANOVA mit Messwiederholung ergibt für die einzelnen Faktoren keine signifikanten Ergebnisse (Bedingung: $F(1,10)=3,20 ; p=0,11 ; \eta^{2}=0,26$; Zeitpunkt: $F(2,10)$ $\left.=1,56 ; p=0,24 ; \eta^{2}=0,15\right)$. In der Interaktion Bedingung $\times$ Zeit zeigt sich jedoch eine Signifikanz (Interaktion Bedingung $\times$ Zeit: $F=5,32 ; p=0,0 \not 2 ; ~{ }^{2}=0,37$ ). Zur Überprüfung dieser Signifikanz wurde ein T-Test (s. Tabelle 7) gerechnet. Hierbei ergibt sich ein signifikanter Unterschied zwischen den Zeitpunkten VR und NR mit einem p-Wert von 0,04. Signifikant unterschiedlich sind ebenfalls die jeweilige Baseline vor Verum- bzw. PlaceboBedingung mit einem p-Wert von unter 0,01 und die beiden Zeitpunkte SP und NP (jeweils unter Placebo-Bedingung) zueinander mit einem p-Wert von 0,01. Die Abbildung 3.1.6-1 zeigt, dass es sich hierbei allerdings um eine signifikante Spielverschlechterung handelt.

Tabelle 6 statistische Auswertung des Arpeggios (Übung 1), die erste Reihe zeigt die Auswertung der zusammengefassten Kriterien Rhythmus, Lautstärke, Klang, ADDS und grobmotorische Bewegungen, die zweite Reihe die gemittelten dystonen und kompensatorischen Bewegungen; gerechnet wurde eine zweifaktorielle ANOVA mit Messwiederholung, $\mathrm{P}=$ Signifikanzniveau

\begin{tabular}{|c|c|c|c|c|c|c|c|c|c|}
\hline \multirow[t]{2}{*}{ ANOVA nur Arpeggio } & \multicolumn{3}{|c|}{ Faktor 1: Bedingung } & \multicolumn{3}{|c|}{ Faktor 2: Zeit } & \multicolumn{3}{|c|}{ Interaktion: Bedingung $\times$ Zeit } \\
\hline & $\mathbf{P}$ & $\mathbf{F}$ & $\eta^{2}$ & $\mathbf{P}$ & $\mathbf{F}$ & $\eta^{2}$ & $\mathbf{P}$ & $\mathbf{F}$ & $\eta^{2}$ \\
\hline $\begin{array}{l}\text { Rhy, Laut, Klang, ADDS } \\
\text { \& grobmot. Bewegungen }\end{array}$ & 0,11 & 3,20 & 0,26 & 0,24 & 1,56 & 0,15 & 0,02 & 5,32 & 0,37 \\
\hline $\begin{array}{lcc}\text { dyst. } \quad \text { \& } & \text { kompensat. } \\
\text { Bewegungen } & \end{array}$ & 0,89 & 0,02 & 0,003 & 0,19 & 1,81 & 0,17 & 0,29 & 1,33 & 0,13 \\
\hline
\end{tabular}

Tabelle 7 statistische Überprüfung des Arpeggios mithilfe eines T-Tests, die Mittelwerte der einzelnen Zeitpunkte aller Probanden wurden miteinander verglichen

\begin{tabular}{|l|l|l|l|l|l|l|l|l|l|}
\hline $\begin{array}{l}\text { T-Test Arpeggio } \\
\text { (zusammengefasste Krit.) }\end{array}$ & VP-VR & NP-NR & SP-SR & NR-VR & SR-VR & SR-NR & NP-VP & SP-VP & SP-NP \\
\hline Signifikanz (2-seitig) & $<0,01$ & 0,61 & 0,71 & 0,04 & 0,10 & 0,78 & 0,73 & 0,01 & 0,10 \\
\hline
\end{tabular}

Abbildung 3.1.6-1 alle Probanden: Arpeggio, zusammengefasste Kriterien Rhythmus, Lautstärke, Klang, ADDS, grobmotor. Bewegungen

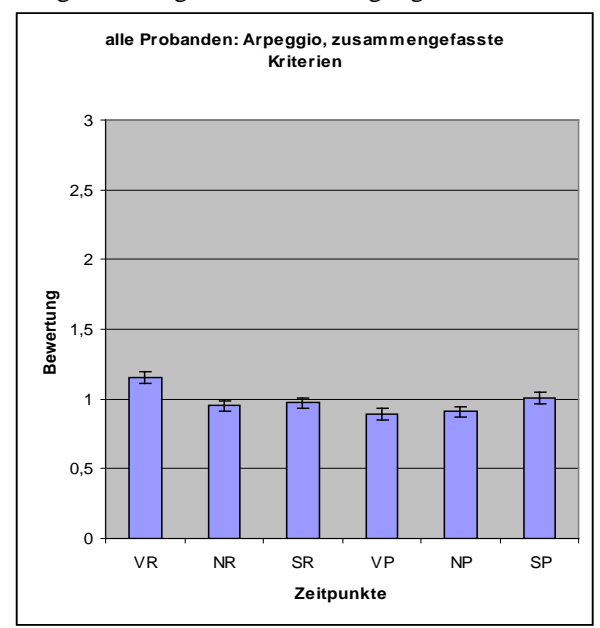

Abbildung 3.1.6-2 alle Probanden: Arpeggio, dystone \& kompensatorische Bewegungen

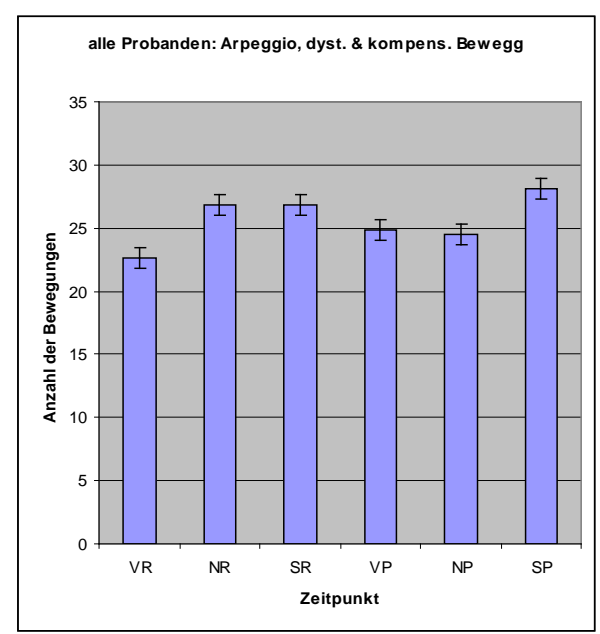

für beide Diagramme gilt: Linie am Ende der Balken zeigt den Standardfehler 
Abbildung 3.1.6-3 Arpeggio unter Verum-Bedingung, VR, NR, SR= Zeitpunkte

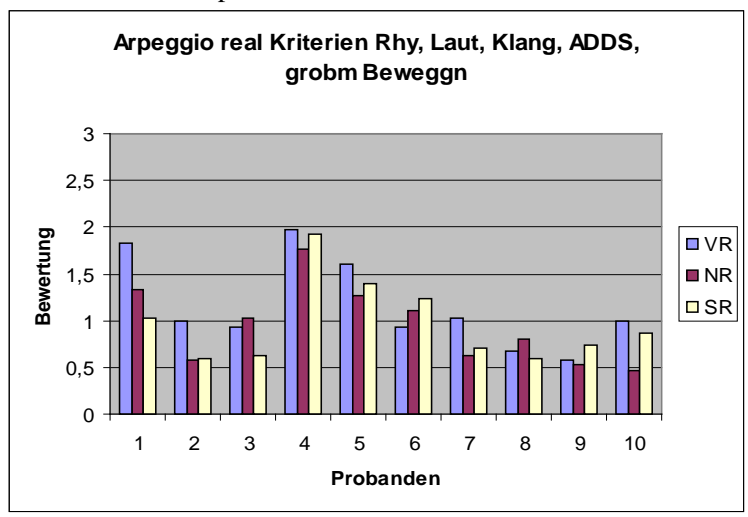

Abbildung 3.1.6-4 Arpeggio unter Placebo-Bedingung, VP, NP, SP= Zeitpunkte

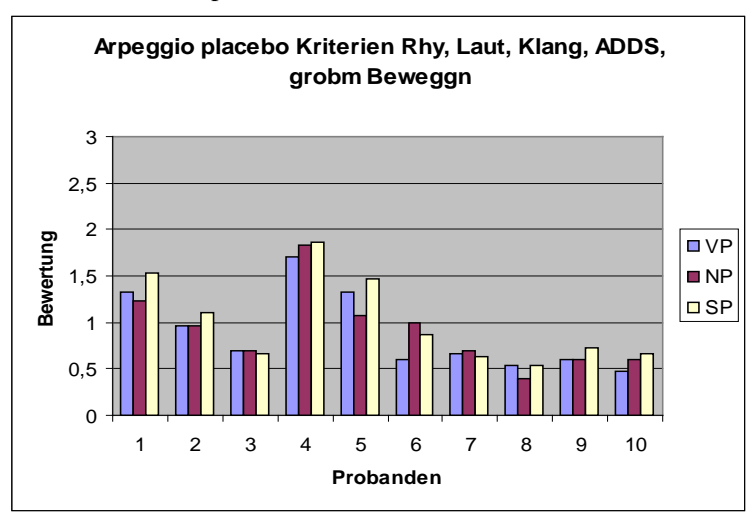

für beide Diagramme gilt: dargestellt ist der Mittelwert aus den Kriterien Rhythmus, Lautstärke, Klang, ADDS und grobmotorische Bewegungen

Die nächsten Diagramme fassen die ausgezählten dystonen und kompensatorischen Bewegungen während des Arpeggios jeweils zu einem Mittelwert zusammen. Hier zeigen sich weder unter Verum- noch unter Placebo-Bedingung Verbesserungen. Wie bereits oben erwähnt, fällt Proband 1 eher durch grobmotorische Bewegungen auf, weshalb er hier so gut wie keine dystonen, bzw. kompensatorischen Bewegungen zeigt. Eine zweifaktorielle ANOVA mit Messwiederholung zeigt entsprechend keine Signifikanz (Bedingung: $F(1,10)=$ 0,$02 ; \mathrm{p}=0,89 ; \eta^{2}=0,003$; Zeitpunkt: $\mathrm{F}(2,10)=1,81 ; \mathrm{p}=0,19 ; \eta^{2}=0,17$; Interaktion Bedingung $\times$ Zeit: $\left.F=1,33 ; p=0,29 ; \eta^{2}=0,13\right)$.

Abbildung 3.1.6-5 dystone und kompensatorische Bewegungen während des Arpeggios unter Verum-Bedingung; VR, NR, SR= Zeitpunkte

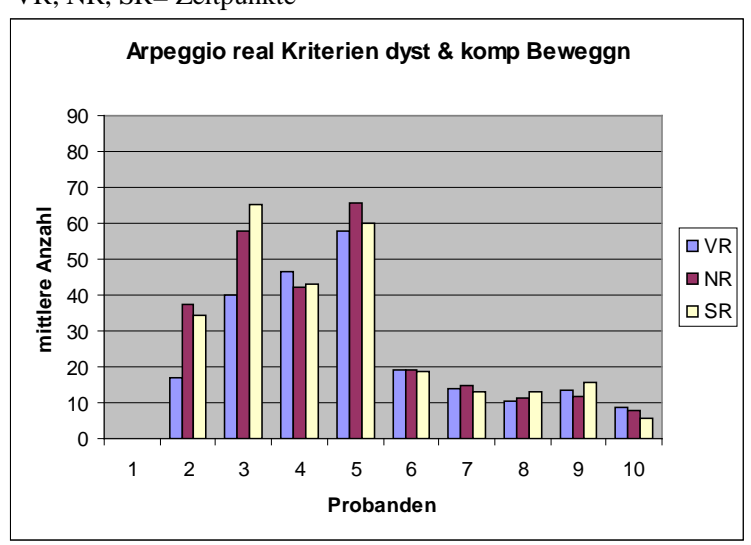

Abbildung 3.1.6-6 dystone und kompensatorische Bewegungen während des Arpeggios unter Placebo-Bedingung, VP, NP, SP= Zeitpunkte

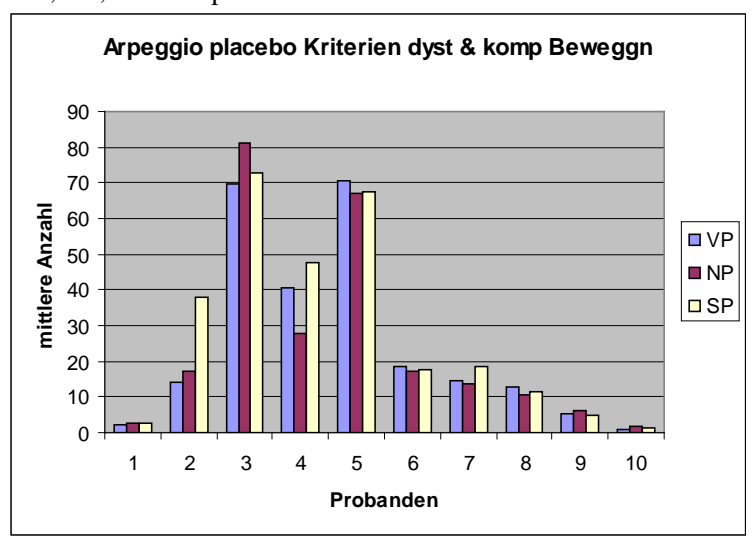

für beide Diagramme gilt: dargestellt ist der Mittelwert aus dystonen und kompensatorischen Bewegungen 


\subsubsection{Zusammenfassung der Ergebnisse}

Betrachtet man den Mittelwert aus allen Probanden zu den unterschiedlichen Zeitpunkten, muss man zusammenfassend sagen, dass es unter Verum-Stimulation keinen deutlichen positiven Effekt auf die Spielfähigkeit gab (s. Abbildung 3.1.7-1). Auch die gemittelte Selbsteinschätzung der Probanden bestätigt dies. Ebenso zeigt die mittlere Anzahl der dystonen und kompensatorischen Bewegungen aller Probanden, dass es unter VerumStimulation zu keinem Rückgang der unphysiologischen Bewegungen gekommen ist (s. Abbildung 3.1.7-3). Lediglich bei den beiden Kriterien Klang und Lautstärke kam es zu einer milden Verbesserung zum Zeitpunkt unmittelbar nach Verum-Stimulation um jeweils 4\% (s. Abbildung 3.1.7-1), diese Verbesserung war aber 60 min nach Stimulation schon nicht mehr auszumachen.

Als einziges signifikantes Ergebnis ergab sich die gesonderte statistische Auswertung der Übung 1, dem Arpeggio. Hier wurden zum einen die Kriterien zu einem Mittelwert zusammengenommen, die mittels einer Likert-Scala (0-3) zu bewerten waren (Rhythmus, Lautstärke, Klang, ADDS \& grobmotorische Bewegungen), zum anderen die Anzahl der dystonen und kompensatorischen Bewegungen beurteilt. Ein gepaarter T-Test ergab für die zusammengefassten Kriterien eine signifikante Verbesserung zwischen den Zeitpunkten vor und unmittelbar nach Stimulation mit einem p-Wert von 0,04. Dagegen steigt aber die mittlere Anzahl der dystonen und kompensatorischen Bewegungen zu denselben Zeitpunkten unter Verum-Bedingung während des Arpeggios von 23 auf 27 an (s. Abbildung 3.1.6-2).

Betrachtet man die Probanden einzeln, war Proband 1 der einzige unter der VerumStimulation profitierende Gitarrist (s.o.). 
Abbildung 3.1.7-1 alle Kriterien unter Verum-Bedingung

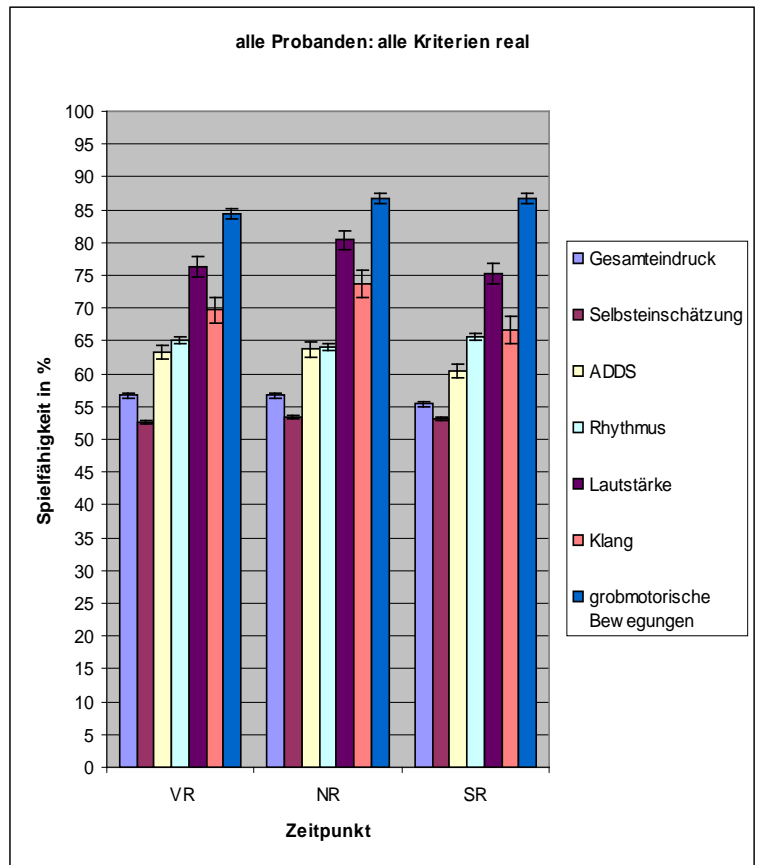

Abbildung 3.1.7-2 alle Kriterien unter Placebo-Bedingung

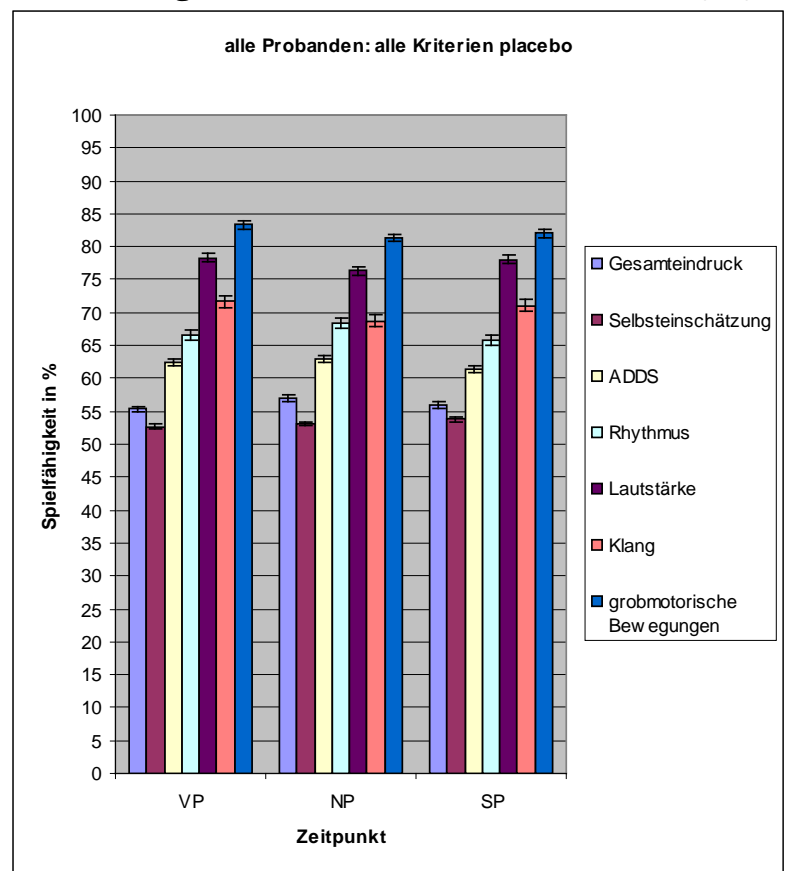

Für die Kriterien Gesamteindruck, ADDS, Rhythmus, Lautstärke, Klang \& grobmotorische Bewegungen erfolgte die Umrechnung der Bewertungsskala 0-3 ( $0=$ keine - 3=deutliche Beeinträchtigung) in eine prozentuale Angabe der Spielfähigkeit anhand der Formel: $\mathrm{y}=(3-\mathrm{x}) * 33,34 ; 100 \%$ bedeutet normales Spielvermögen, ohne jegliche Beeinträchtigung; Linie am Ende der Balken zeigt den Standardfehler

Abbildung 3.1.7-3 Mittlere Anzahl dystoner und kompensatorischer Bewegungen unter Verum-Bedingung

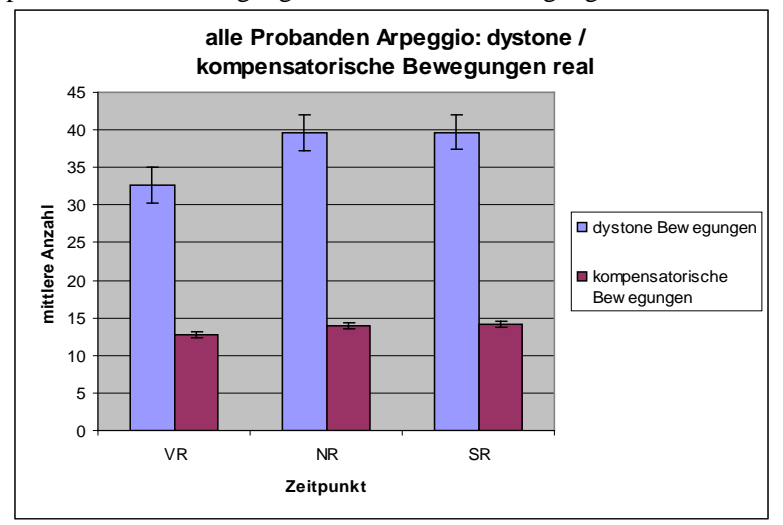

Abbildung 3.1.7-4 Mittlere Anzahl dystoner und kompensatorischer Bewegungen unter Placebo-Bedingung

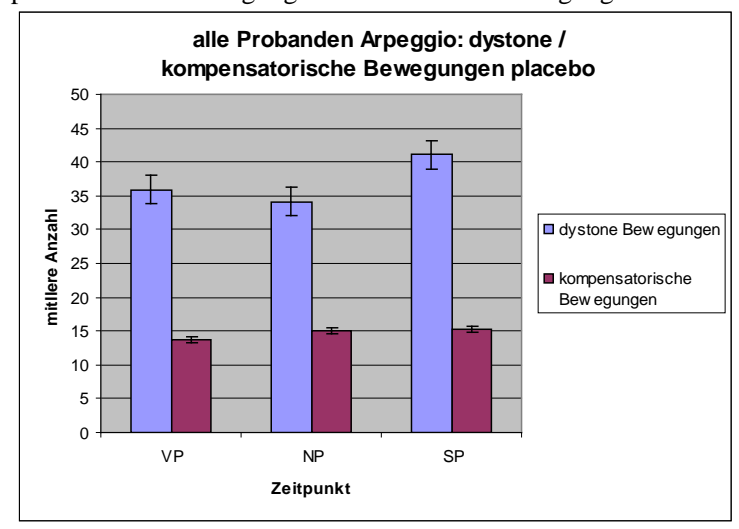

für beide Diagramme gilt: Linie am Ende der Balken zeigt den Standardfehler

\subsection{Kumulative Übezeit}

Wie schon im Methodikteil erwähnt, haben wir in unserem Fragebogen die kumulative Übezeit in den jeweiligen Altersabschnitten erfragt. Bei der Auswertung und graphischen Darstellung fällt auf, dass sich bei sechs Probanden (Proband 2, 4, 5, 6, 7 und 9; s. Abbildungen 3.1.7-2, -4, -5, -6, -7 und -9) die fokale Dystonie während oder unmittelbar im Anschluss an eine Phase intensiven Übens entwickelt hat. 
Abbildung 3.1.7-1

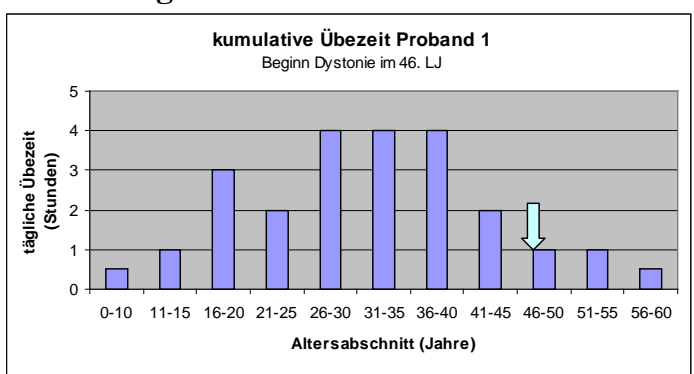

\section{Abbildung 3.1.7-3}

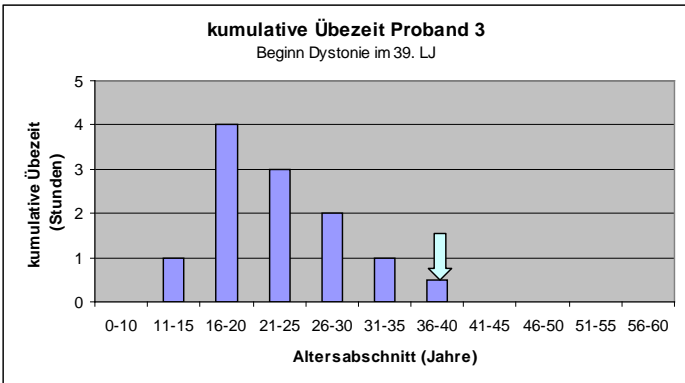

Abbildung 3.1.7-5

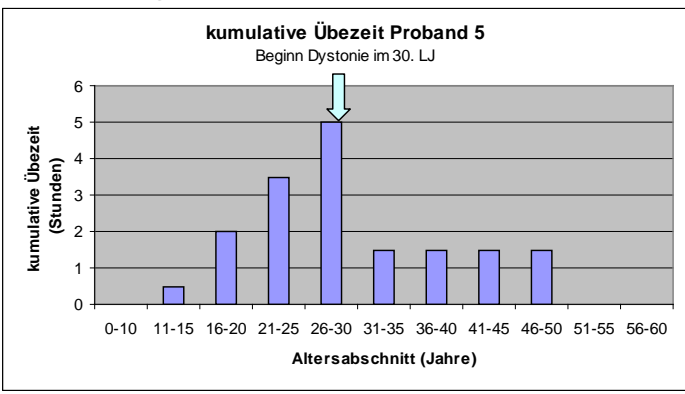

Abbildung 3.1.7-7

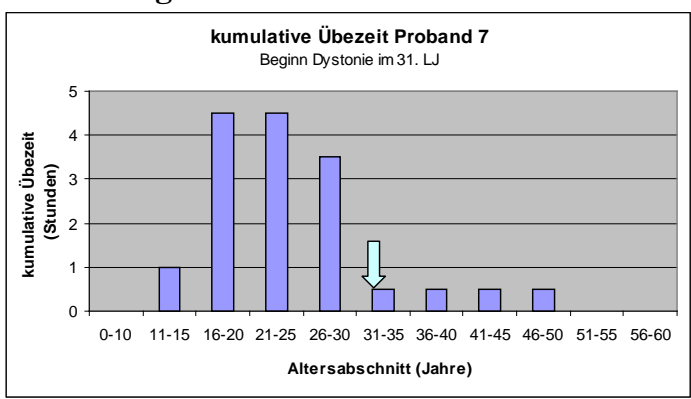

\section{Abbildung 3.1.7-9}

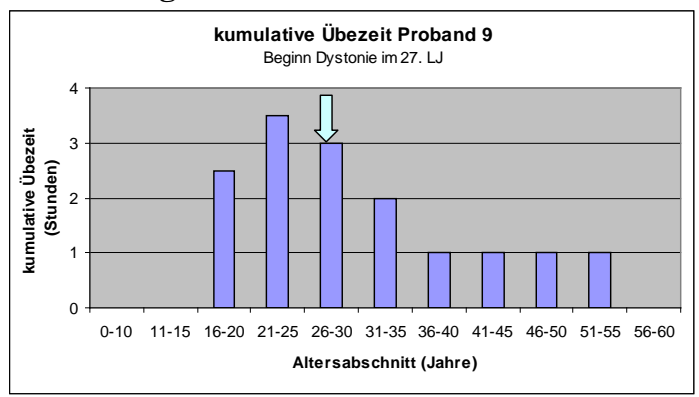

Abbildung 3.1.7-2

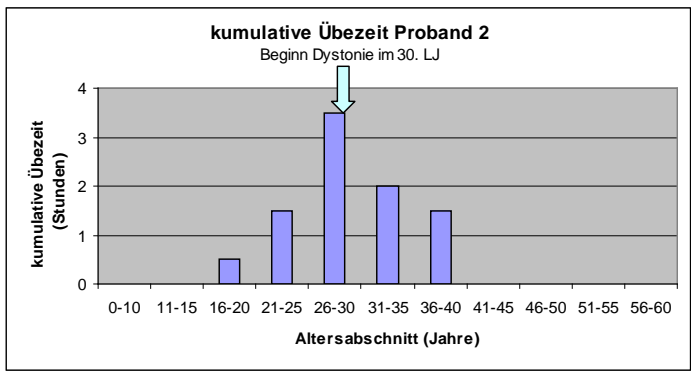

\section{Abbildung 3.1.7-4}

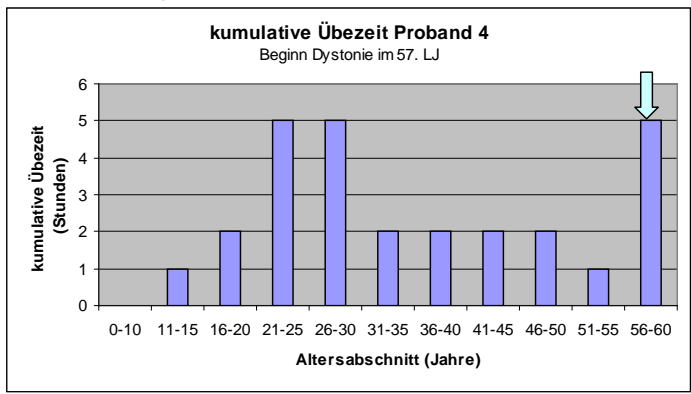

\section{Abbildung 3.1.7-6}

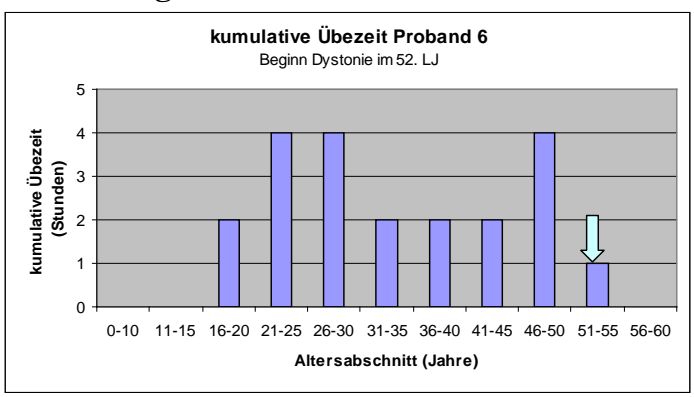

Abbildung 3.1.7-8

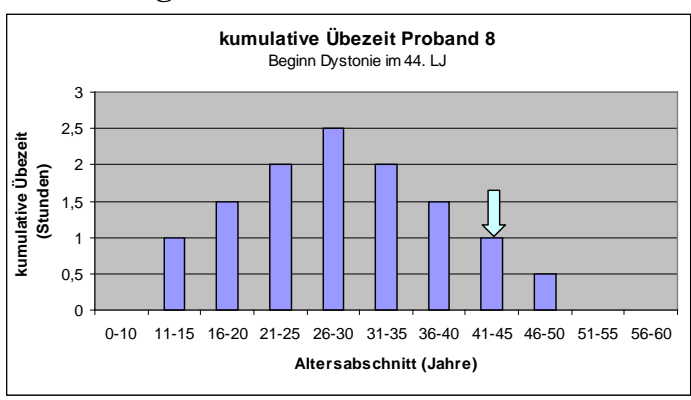

Abbildung 3.1.7-10

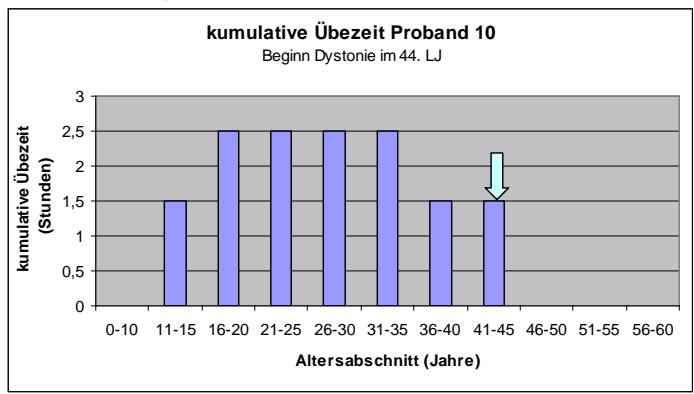




\subsection{State-Trait-Angstinventar}

Die Auswertung der STAI-Fragebögen ergibt die in Tabelle 8 ersichtlichen Werte. Die StateWerte eignen sich nur zum Vergleich der Intensität eines emotionalen Zustands zu den jeweiligen Terminen. Der Zustand ist gekennzeichnet durch Anspannung, Besorgtheit, Nervosität, innere Unruhe und Furcht vor zukünftigen Ereignissen. Ein Wert von 20 entspricht dem Nichtvorhandensein, ein Wert von 80 der maximalen Intensität des Gefühls (Laux et al. 1981). Die State-Werte sind ausnahmslos kleiner oder gleich dem StateErwartungswert 37,33 (SD 9,83). Dieser Erwartungswert beruht auf einer Eichstichprobe von 740 Männern im Alter von 30-59 Jahren, also entsprechend unseren Probanden (mittleres Alter: 48,8 Jahre \pm 6,43; MIN-MAX: 39-57 Jahre).

Der Trait-Wert, also Ängstlichkeit als generelle Eigenschaft, ist unter Berücksichtigung des 95-prozentigen Konfidenzintervalls („wahrer“ Trait-Wert) bei der Hälfte der Probanden (Proband 1, 2, 6, 7 \& 8) höher als der entsprechende Trait-Erwartungswert von 34,59 (SD 8,92). Wie bei dem State-Wert entstammt dieser Trait-Erwartungswert ebenfalls einer Eichstichprobe von 740 Männern im Alter von 30-59 Jahren. Der mittlere Trait-Wert unserer zehn Probanden liegt bei 40,7 (SD 7,89; MIN-MAX: 32-54, „wahrer“ TraitWert: 35,3 - 46,2), womit er unter Berücksichtigung des 95-prozentigen Konfidenzintervalls ebenfalls signifikant erhöht ist.

Tabelle 8 State- und Trait-Werte unserer Probanden

\begin{tabular}{|l|l|l|l|l|}
\hline Proband & $\begin{array}{l}\text { State } \\
\text { 1. Termin }\end{array}$ & $\begin{array}{l}\text { State } \\
\text { 2. Termin }\end{array}$ & Trait & "wahrer“ $_{\text {Trait-Wert }^{+}}$ \\
\hline 1 & $*$ & 39 & 46 & $40,5-51,5$ \\
\hline 2 & 37 & 37 & 46 & $40,5-51,5$ \\
\hline 3 & $*$ & 34 & 32 & $26,5-37,5$ \\
\hline 4 & 37 & 30 & 32 & $26,5-37,5$ \\
\hline 5 & $*$ & 34 & 33 & $27,5-38,5$ \\
\hline 6 & 37 & 31 & 49 & $43,5-54,5$ \\
\hline 7 & 34 & 32 & 53 & $47,5-58,5$ \\
\hline 8 & 28 & 28 & 44 & $38,5-49,5$ \\
\hline 9 & 31 & 36 & 33 & $27,5-38,5$ \\
\hline 10 & 34 & 31 & 39 & $33,5-44,5$ \\
\hline Mittelwert & $\mathbf{3 4}$ & $\mathbf{3 3 , 2}$ & $\mathbf{4 0 , 7}$ & $\mathbf{3 5 , 2}-\mathbf{4 6 , 2}$ \\
\hline MIN & 28 & 28 & 32 & \\
\hline MAX & 37 & 39 & 54 & \\
\hline SD & 3,46 & 3,43 & 7,89 & \\
\hline
\end{tabular}

Erwartungswerte (Männer, N=740, 30-59 Jahre): State 37,33 (SD 9,83),

Trait 34,59 (SD 8,92); ${ }^{+}$unter Berücksichtigung der Reliabilität des Messinstrumentes (des Fragebogens) liegt der wahre

Testwert mit 95prozentiger Wahrscheinlichkeit in diesem Intervall; grau hinterlegte Werte weichen signifikant vom Erwartungswert ab; * State vom 1 . Termin fehlt 


\section{Diskussion}

Ausgangspunkt unserer Studie war die Annahme, dass sich hinter der Pathophysiologie von Musikern mit fokaler Dystonie eine mangelnde Inhibition auf verschiedenen Ebenen im ZNS (somatosensorischer und motorischer Kortex, Basalganglien) verbirgt. Unter dieser Annahme wollten wir herausfinden, ob betroffene Musiker von einer kathodalen tDCS über dem primärmotorischen Kortex, und damit einer Verminderung der Erregbarkeit dieses Areals, profitieren.

\subsection{Fehlender Effekt von tDCS}

Betrachtet man die zehn Probanden insgesamt, stellte sich unter kathodaler tDCS keine Verbesserung der Spielfähigkeit ein. Innerhalb der einzelnen Beurteilungskriterien ergaben sich unter Stimulation keine signifikanten Effekte. Auch die Anzahl der dystonen und unphysiologisch kompensatorischen Bewegungen nahm unter Stimulation nicht ab. Bei gesonderter Betrachtung der Probanden, die erst seit bis zu zwei Jahren unter der Dystonie litten, ließen sich ebenfalls keine Effekte feststellen. Was kann der Grund hierfür sein?

Der vorliegende Versuch, tDCS bei Musikern mit fokaler Dystonie, stellt eine Pilot-Studie dar. Kriterium für die Auswahl der Methode war unter anderem auch die Zumutbarkeit für die betroffenen Musiker. TDCS stellte eine gute Methode auch wegen ihrer einfachen Handhabung dar: oberflächliche Elektroden, die sehr geringen Stromstärken, bei denen höchstens ein leichtes Kribbeln wahrzunehmen ist, ein handlicher Gleichstrom-Generator.

Eine Reihe möglicher Gründe kann die fehlenden Effekte der tDCS auf die Symptomatik der Patienten bedingt haben. Ein möglicher Kritikpunkt ist das Aufsuchen des primärmotorischen Kortex M1 in der aktuellen Studie. Die korrekte Identifikation des kortikalen Zielareals ist für die optimale Positionierung der Stimulationselektrode entscheidend. Wir haben den primärmotorischen Kortex anhand verschiedener Fixpunkte am Schädel aufgesucht (Articulatio temporomandibularis, Nasion und Inion). Eine Studie von Steinmetz und Mitarbeitern hat bereits Ende der Achtziger Jahre (Steinmetz et al. 1989) die Genauigkeit des Lokalisierens bestimmter Hirnareale anhand des auch von uns verwendeten 10-20-Systems mittels MRT-Aufnahmen überprüft. Bestimmt wurde unter anderem die Lage des Sulcus centralis. Anterior-posteriore Variationen der Lage des Sulcus centralis lagen im Mittel von 16 gesunden Probanden innerhalb von $2 \mathrm{~cm}$. Die Bestimmung der Hirnareale anhand von externen Fixpunkten am Schädel hielt man für vertretbar. Untersucht wurden allerdings 
ausschließlich gesunde Probanden. Möglicherweise ist aber auch die Repräsentation des motorischen Kortex bei der Musikerdystonie verändert.

Eine genauere Methode wäre die Bestimmung des primärmotorischen Kortex mittels TMS. Bei festgelegter Reizintensität einer Magnetspule wird hierbei der Ort über dem primärmotorischen Kortex aufgesucht, an dem die von den Zielmuskeln abgeleiteten MEPs die größte Amplitude haben. Ein TMS-Impuls ist aber im Vergleich zu tDCS deutlich unangenehmer. Dieses Verfahren schien uns für unsere Probanden zu abschreckend zu sein, weswegen wir uns für o.g. Methode entschieden haben.

Die fokale Dystonie ist eine sehr stabile Bewegungsstörung. Möglicherweise reicht eine einmalige 20-minütige Stimulation nicht aus, um therapeutisch relevante Veränderungen motorischer Funktionen zu erzeugen. Hiermit vereinbar sind Beobachtungen, die bei einem neueren neurochirurgischen Therapieansatz, der Tiefenhirnstimulation, gemacht wurden. Dies ist ein Verfahren, bei dem intraoperativ eine Elektrode im Globus pallidus internus, einem Teil der Basalganglien, eingebracht wird. Man weiß, dass eine abnormale Aktivität des Globus pallidus internus mit der Dystonie zusammenhängt (Collins et al. 2010), je geringer die Feuerrate, desto stärker scheint die Dystonie ausgeprägt zu sein. In einer Studie von Ruge et al. (Ruge et al. 2009) untersuchte man die Effekte, die durch Tiefenhirnstimulation bei Patienten mit primärer Dystonie erzielt wurden. Messparameter, um Effekte zu beurteilen, waren neben anderen die short-latency intracortical inhibition (SICI) als ein Kriterium der GABAergen Erregbarkeit. Diese war vorab erniedrigt. Ergebnis war, dass sich bei primären Dystonieformen die SICI unter Tiefenhirnstimulation auf ein normales Niveau bringen ließ und sich hierunter die klinische Symptomatik der Patienten verbesserte. Diese Verbesserung stellte sich aber oft erst nach einer Latenz von 3-6 Monaten ein.

Monte-Silva et al. (Monte-Silva et al. 2010) veröffentlichten eine Studie, in der sie geeignete kathodale tDCS-Protokolle suchten, mithilfe derer ein Maximum an Nacheffekten erzielt werden kann. Es wurde repetitiv stimuliert, wobei die Pause zwischen den einzelnen Applikationen variiert wurde. Es zeigte sich, dass der richtige Zeitpunkt einer wiederholten Stimulation von besonderer Bedeutung ist. Wird ein zweites Mal innerhalb der Nacheffekte einer ersten kathodalen tDCS stimuliert, lassen sich hierdurch sowohl Dauer als auch Stärke der Nacheffekte nach der zweiten Stimulation verlängern, bzw. vergrößern. Dies wäre ein interessanter Ansatz, um zu untersuchen, ob eine längerfristige kathodale tDCS bei Musikern mit Dystonie einen positiven Effekt bringt.

Ein anderer Grund für das Ausbleiben eines Effektes kann die Komplexität des Gitarrespiels sein, wodurch eine ausschließliche Stimulation des primärmotorischen Kortex M1 
möglicherweise unzureichend wäre. Das Spielen eines Instrumentes ist eine motorisch anspruchsvolle Aufgabe für das Gehirn, die nicht nur den primärmotorischen Kortex, sondern auch andere Areale, wie den prämotorischen und supplementärmotorischen Kortex mit einbezieht. Hier werden Bewegungsabläufe geplant, deren Abfolge festgelegt und das Zusammenspiel der beiden Hände koordiniert (Altenmüller 2003). In einer Studie von Boros et al. (Boros et al. 2008) konnte gezeigt werden, dass auch tDCS über dem prämotorischen Kortex in der Lage ist, die Erregbarkeit des ipsilateralen primärmotorischen Kortex M1 zu beeinflussen. Allerdings konnten unter motorischer Ruhebedingung Effekte nur unter anodaler tDCS des prämotorischen Kortex erzielt werden. Hierbei wurde die intrakortikale Inhibition (SICI) gesenkt. Die Beeinflussung der Erregbarkeit von M1 durch Stimulation des prämotorischen Kortex verdeutlicht die enge kortiko-kortikale Verbindung der beiden Hirnareale.

Denkbar ist auch, dass kathodale tDCS nicht in der Lage ist, den Mangel an Inhibition oder anders ausgedrückt, den Überschuss an kortikaler Erregbarkeit speziell bei der Musikerdystonie (MD) ausreichend zu senken. Zu den Unterschieden der MD im Vergleich zum Schreibkrampf haben Rosenkranz et al. (Rosenkranz et al. 2005) eine interessante Studie herausgebracht. Dabei untersuchten sie die Interaktion eines sensiblen Reizes auf die motorische Erregbarkeit. Vibration wurde als sensibler Reiz an einem einzelnen intrinsischen Handmuskel gesetzt, gleichzeitig wurde die motorische Erregbarkeit via TMS des stimulierten Muskels und anderer Handmuskeln gemessen. Messungen wurden durchgeführt am ersten Musculus interosseus dorsalis (FDI), am Musculus abductor pollicis brevis (APB) und am Musculus abductor digiti minimi (ADM). Dabei liegen der FDI und der APB anatomisch sehr nah zu einander, der ADM sehr weit von den anderen beiden entfernt. Bei Patienten mit Schreibkrampf hatte die Vibration nur einen sehr kleinen Effekt auf die motorische Erregbarkeit. Bei Musikern mit FD dagegen war der Einfluss des sensiblen Reizes sehr groß, aber auch sehr unfokussiert. Neben den MEPs als Maß der motorischen Erregbarkeit wurde auch die intrakortikale Inhibition (SICI) gemessen. Von ihr wird vermutet, dass sie von GABAergen Synapsen innerhalb des motorischen Kortex erzeugt wird (Ziemann et al. 1996). Es stellte sich heraus, dass bei Patienten mit Schreibkrampf unter sensibler Stimulation eines Handmuskels die SICI aller Handmuskeln nahezu unverändert blieb. Bei der MD dagegen war die SICI unter sensibler Stimulation in allen Handmuskeln deutlich reduziert. Und zwar nicht nur, wie in einer Kontrollgruppe gesunder Musiker (hier war die SICI nur in korrespondierenden Muskeln wie dem FDI und dem APB vermindert), sondern auch in einem weit entfernt liegendem Muskel, wie dem ADM (passend zu dem 
Modell von Hallet, dem Verlust der surround-inhibition). Möglicherweise erfordert dieser ausgeprägte Verlust an SICI bei Musikern mit FD ein höheres Maß an Reduzierung des Überschusses kortikaler Erregbarkeit, als durch unsere einzelne kathodale tDCS erfolgt ist.

\subsection{Verbesserung der Spielfähigkeit bei Proband 1}

Zwar hat kathodale tDCS bei Betrachtung aller Probanden keinen signifikanten Effekt gebracht, ein einzelner Proband (Proband 1) konnte aber von der Stimulation profitieren.

Die Besonderheit des Probanden 1 ist seine Form der Dystonie. Es ist keine fokale Dystonie einzelner Finger, die sich durch dystone bzw. kompensatorische Bewegungen der betroffenen Finger äußern würde. Es ist eine Dystonie des Armes, insbesondere des Unterarmes, die sich in grobmotorischen Bewegungen des Unterarmes und der gesamten Hand äußert. Der Patient spielt insgesamt mit deutlich erhöhtem Muskeltonus. Die gesamte Hand hat die Tendenz, vom Gitarrenkorpus wegzuziehen, wogegen vom Probanden aktiv gegengesteuert werden muss. Sehr stark werden gleichzeitig funktionelle Muskelagonisten und -antagonisten aktiviert. Dies zeigt sich in ausgeprägt grobmotorischen Bewegungen.

Der Gesamteindruck verbesserte sich unter Stimulation um 34\%, die grobmotorischen Bewegungen verbesserten sich um 10\% (s. Abbildung 3.1.4-1). Wie ist dieses Ergebnis einzuschätzen?

Kann es durch die Einnahme eines Betablockers (Propanolol) zu einer Effektverstärkung gekommen sein? Den Betablocker nahm der Proband, um seinen generell erhöhten Muskeltonus etwas herabzuregulieren. In einer Studie untersuchten Nitsche et al. (Nitsche et al. 2004a) den Einfluss von u.a. adrenergen Substanzen auf die durch tDCS verursachten kortikalen Erregbarkeitsveränderungen. Die Probanden nahmen zwei Stunden vor Stimulationsbeginn 80mg Propanolol ein. Ergebnis war, dass der Betablocker die Dauer der Nacheffekte sowohl nach anodaler als auch nach kathodaler tDCS verkürzte. Eine Effektverstärkung kann in unserem Fall also ausgeschlossen werden.

Möglicherweise liegt bei diesem Probanden eine etwas andere Pathophysiologie zugrunde. Neben der tätigkeitsspezifischen Dystonie fällt der generell erhöhte Muskeltonus beim Gitarrespiel auf. Insgesamt liegt also eine etwas globalere Bewegungsstörung vor. Die Stimulation über die $35 \mathrm{~cm}^{2}$ großen Elektroden ist vermutlich auch nicht nur fokussiert auf die von der FD betroffenen motorischen Repräsentationsareale, sondern eher global, größere Anteile des primärmotorischen Kortex betreffend. Möglicherweise lässt sich hierdurch der Effekt durch die Stimulation bei dem Probanden 1 erklären. 
In unserer Studie ist es nur ein einzelner Proband, der profitiert hat. Vielleicht können weitergehende Studien mit tDCS speziell bei Musikern mit dieser besonderen wenig fokalen Form der Dystonie weitere Erkenntnisse bringen.

\subsection{Gesteigerte Ängstlichkeit}

Die Erhebung der Ängstlichkeit unserer zehn Probanden nach dem State-Trait-Angstinventar nach Laux und Mitarbeitern ergab, dass die Ängstlichkeit als generelle Eigenschaft (Trait) gegenüber einer der Normalbevölkerung entsprechenden Eichstichprobe erhöht ist (mittlerer Trait-Wert unserer Probanden: 40,7; mittlerer Trait-Wert der Eichstichprobe: 34,6).

Dieses Ergebnis unterstreicht die Erkenntnisse von Jabusch und Altenmüller (Jabusch und Altenmüller 2004, Jabusch et al. 2004b). In den damaligen Studien zeigte sich, dass Musiker mit FD häufiger unter verschiedenen Formen von Phobien leiden (insbesondere Klaustrophobie, Akrophobie (Höhenangst) oder sozialer Phobie). Anhand verschiedener Fragebögen (Freiburg Personality Inventory, Questionnaire for Competence and Control Orientations) wurden Ängstlichkeit und Perfektionismus abgefragt. Insbesondere wurde zwischen den Zeitpunkten vor Erkrankung und nach Erkrankung differenziert. Gegenüber einer gesunden Kontrollgruppe zeigte sich, dass Patienten mit fokaler Dystonie signifikant häufiger schon vor Erkrankungsbeginn vermehrte Ängstlichkeit aufwiesen.

Diese erhöhte Ängstlichkeit spielt eine Rolle bei der Entwicklung von FD (s. Teil 1.1.1. Epidemiologie und Ätiologie Abb. 1). Dystone Bewegungen werden von Musikern mit gesteigerter Ängstlichkeit und Perfektionismus zu Beginn der Erkrankung als besonders störend und beängstigend wahrgenommen. Dieser emotionale Stress bedingt eine Aktivierung der Amygdala, die ihrerseits Einfluss auf den primärmotorischen Kortex im Sinne eines gedächtnissteigernden Effekts nimmt. Dadurch kommt eine emotionsbedingte stärkere Einprägung dystoner Bewegungen bei Musikern mit gesteigerter Ängstlichkeit zustande. Unsere Musiker bringen also einen intrinsischen Triggerfaktor, nämlich den der gesteigerten Ängstlichkeit mit sich.

Zwei Dinge gilt es allerdings bei diesem Ergebnis zu berücksichtigen. Zum einen ist es nur eine sehr kleine Anzahl an Probanden, aus denen wir einen Mittelwert gebildet haben. Zum anderen sind es gleich zwei Kriterien, die unsere Probanden von der Normalbevölkerung unterscheiden: erstens die Dystonie, zweitens die Eigenschaft als Musiker. Ganz korrekt wäre es also gewesen, hätte man den Trait-Wert unserer Probanden mit einer Kontrollgruppe aus gesunden Musikern verglichen. 


\subsection{Ausblick}

Auf was sollten nun also zukünftige Studien abzielen, was könnte an der tDCS verändert, bzw. verbessert werden, um mögliche therapeutische Effekte bei Patienten mit Musikerdystonie zu erzielen?

Die Effektivität einer Stimulation sollte zukünftig auch in den klinischen Studien neurophysiologisch mittels TMS und den dadurch abgeleiteten MEPs überprüft werden. So könnte die korrekte Position der Stimulationselektrode kontrolliert und eine unmittelbare Auswirkung auf die kortikale Erregbarkeit überprüft werden.

Die Stimulation könnte fokussierter, insbesondere auf den dystonen Finger zielend, erfolgen. Nitsche und Mitarbeiter haben dazu eine Studie herausgebracht (Nitsche et al. 2007). Neben einer tDCS mit zwei $35 \mathrm{~cm}^{2}$ großen Elektroden, stimulierten sie auch mit einer kleineren 3,5 $\mathrm{cm}^{2}$ großen Stimulationselektrode. Um die gleiche Stromdichte von $0,03 \mathrm{~mA} / \mathrm{cm}^{2}$ wie bei üblicher Elektrodengröße zu erzeugen, wurde hierbei die Stromstärke auf 0,1 mA reduziert. Auch mit reduzierter Elektrodengröße konnten gleiche Effekte während und nach der Stimulation erzeugt werden. MEPs, die an zwei verschiedenen Handmuskeln abgeleitet wurden, zeigten aber, dass die Stimulation mit der kleineren Elektrode fokussierter ist. Es wurden durch TMS induzierte MEPs am Musculus interosseus dorsalis (FDI) und am Musculus abductor digiti minimi (ADM) abgeleitet. Während die $35 \mathrm{~cm}^{2}$ große Elektrode beide Repräsentationsareale über dem primärmotorischen Kortex abdeckte, wurde bei Stimulation mit der kleineren 3,5 $\mathrm{cm}^{2}$ großen Elektrode nur das Repräsentationsareal des ADM erfasst, das Repräsentationsareal des FDI lag außerhalb. Bei Stimulation mit der üblichen $35 \mathrm{~cm}^{2}$ großen Elektrode veränderten sich die MEPs beider Muskeln signifikant. Bei Stimulation mit der kleineren 3,5 $\mathrm{cm}^{2}$ großen Elektrode wirkte sich die Stimulation nur auf den ADM aus, die MEPs des FDI blieben unverändert. Möglicherweise brächte eine auf den dystonen Finger fokussierte kathodale tDCS einen besseren Effekt.

Die Erfahrung aus der Tiefenhirnstimulation bei Patienten mit primärer Dystonie zeigt, dass klinische Effekte erst nach einer Latenzzeit von 3-6 Monaten auftreten. Übertragen auf die tDCS hieße das, dass sich auch hier klinische Effekte eventuell erst nach deutlich längerer Stimulationszeit einstellen. Hilfreich hierbei wäre bereits oben erwähnte Verlängerung der Nacheffekte durch repetitive Stimulationsprotokolle (Monte-Silva et al. 2010).

Eine weitere Möglichkeit zur Verlängerung der Nacheffekte besteht in der Kombination der tDCS mit Medikamenten. Kuo et al. haben hierzu eine interessante Studie herausgebracht (Kuo et al. 2008). TDCS wurde mit der Dopamin-Vorstufe L-Dopa kombiniert. Anhand elf gesunder Probanden wurde der Effekt u.a. in Kombination mit kathodaler Stimulation 
untersucht. Eine Stunde vor Stimulation nahmen die Probanden 100 mg L-Dopa zu sich. Es stellte sich heraus, dass der inhibitorische Nacheffekt der kathodalen tDCS um den Faktor 20 verlängert werden konnte. Durch MEPs erhobene Nacheffekte, die unter alleiniger Stimulation bis zu 120 min nachweisbar waren, hielten bei Kombination mit L-Dopa bis zum nächsten Morgen nach Stimulation an. Es wird vermutet, dass intrazerebrale D2-Rezeptoren an dem Plastizität stabilisierenden Effekt beteiligt sind.

Vergessen darf man auch nicht die Kombination mit den verschiedenen ebenso sehr effektiven Retraining-Methoden wie z.B. dem „Sensory-Motor-Retuning“ nach Candia oder dem speziell entwickelten Retraining nach Boullet (s. Therapie der Musikerdystonie). Solche Retraining-Methoden ziehen sich oft über viele Monate hin und erfordern von dem betroffenen Musiker eine besondere Disziplin. Candia und Mitarbeiter konnten zeigen, dass sich nach erfolgreichem Absolvieren des „Sensory-Motor-Retunings“ der somatosensorische Kortex der von der FD betroffenen Musiker reorganisiert hatte (Candia et al. 2003). Die bei der FD typischen Verschmelzungen einzelner somatosensorischer Repräsentationsareale der Finger bildeten sich zurück, der somatosensorische Kortex rehabilitierte immer mehr im Sinne eines somatotopisch normalen Homunculus. Weitere Studien könnten untersuchen, ob z.B. eine anodale tDCS einen begünstigenden Effekt auf das Neuerlernen von nicht-dystonen Bewegungen hat. Hierbei stände ein dadurch gesteigertes Knüpfen von Aufgaben-relevanten Synapsen in der Phase des Erlernens im Vordergrund (Antal et al. 2004b). Eventuell könnten hierdurch bleibende Erfolge durch eine Kombination mit tDCS schneller herbeigeführt werden.

Abschließend kann man zusammenfassen, dass sich in unserer Pilotstudie unter einmaliger 20-minütiger kathodaler tDCS bei Gitarristen mit FD keine spielverbessernden Effekte zeigten. Es gibt aber durchaus verschiedene Ansätze, eine solche Stimulation zu optimieren, um eventuell in Zukunft relevante Effekte erzielen zu können. 


\section{Zusammenfassung}

Die Musikerdystonie ist eine aufgabenspezifische Bewegungsstörung, die in einer schmerzlosen Beeinträchtigung der Feinmotorik besteht. Häufig tritt sie nach Phasen intensiven Übens auf. Bei der Pathophysiologie der FD geht man von einem Mangel an Inhibition auf verschiedenen Ebenen des ZNS aus. Neben einer Übererregbarkeit des motorischen Kortex, erschwert eine mangelnde surround-inhibition (Hallett 2004) auf Ebene der Basalganglien das Ausführen von präzisen Bewegungen. Eine gestörte Homöostase (Siebner et al. 2004, Quartarone et al. 2005) bedingt eine überschießende kortikale Erregbarkeit außerhalb eines physiologisch sinnvollen Rahmens. TDCS ist ein nicht invasives Verfahren zur Erzeugung von Neuroplastizität über dem motorischen Kortex. Durch kathodale Stimulation wird das Ruhemembranpotential herabgesetzt und die Erregbarkeit dadurch vermindert. Eine Studie von Antal et al. (Antal et al. 2004b) über visuo-motorisches Lernen bei gesunden Probanden zeigte, dass kathodale tDCS über dem visuellen Kortex V5 einen begünstigenden Effekt hat, wenn eine Aufgabe bereits „überlernt“ ist. Wahrnehmung und Ausführung wurden fokussiert. Wir gingen der Frage nach, ob eine kathodale tDCS über dem primärmotorischen Kortex bei Gitarristen mit FD in der Lage ist, gesteigerte kortikale Erregbarkeit (mangelnde Inhibition) zu reduzieren und damit eine fokussiertere neuronale Aktivität zu ermöglichen. Wir applizierten zehn von der FD betroffenen professionellen Gitarristen eine kathodale tCDS (2mA, 20 min) über dem primärmotorischen Kortex kontralateral zu der betroffenen Hand. Standardisierte Übungen mussten zu verschiedenen Zeitpunkten (vor, unmittelbar nach und 60 min nach Stimulation) gespielt und sowohl subjektiv als auch objektiv beurteilt werden. Die objektive Beurteilung erfolgte durch drei unabhängige Rater, die Videomitschnitte der einzelnen Probanden anhand verschiedener Kriterien $\mathrm{zu}$ beurteilen hatten. Die statistische Auswertung erfolgte mithilfe einer zweifaktoriellen Varianzanalyse (Faktor 1: Stimulationsbedingung, Faktor 2: Zeitpunkt) und einem gepaarten T-Test. Betrachtet man den Mittelwert aus allen Probanden zu den unterschiedlichen Zeitpunkten, muss man zusammenfassend sagen, dass es unter kathodaler tDCS keinen deutlichen positiven Effekt auf die Spielfähigkeit gab (s. Abbildung 3.1.7-1). Auch die gemittelte Selbsteinschätzung der Probanden bestätigt dies. Betrachtet man die Probanden einzeln, war Proband 1 der einzige unter kathodaler tDCS profitierende Gitarrist. Ihn unterscheidet eine weniger fokale, den gesamten Unterarm betreffende Form der Dystonie von den übrigen Probanden. Neben den Ergebnissen zur kathodalen tDCS ergab die Erhebung 
des State-Trait-Angstinventars eine gesteigerte Ängstlichkeit (Trait) unserer zehn Probanden gegenüber der Normalbevölkerung. Dies bestätigt die Ergebnisse früherer Studien von Altenmüller und Jabusch (Jabusch und Altenmüller 2004, Jabusch et al. 2004b), wonach eine gesteigerte Ängstlichkeit ein Triggerfaktor bei der Entwicklung einer FD ist. Auch die Erhebung der kumulativen Übezeit zeigt, dass der Zeitpunkt des Auftretens der FD häufig während oder unmittelbar nach Phasen sehr intensiven Übens ist. Gründe für das Ausbleiben eines Effektes unter kathodaler tDCS könnten möglicherweise eine nicht ausreichend genaue Lokalisierung des primärmotorischen Kortex oder die relativ große Stabilität der Bewegungsstörung, für dessen Beeinflussung eine einmalige 20-minütige tDCS nicht ausreicht, sein. Zukünftige tDCS-Protokolle könnten repetitiv über einen längeren Zeitraum und eventuell in Kombination mit Nacheffekt-verlängernden Medikamenten erfolgen. Eine anodale tDCS könnte in Kombination mit Retraining-Methoden einen begünstigenden Effekt auf das Neuerlernen von nicht-dystonen Bewegungen haben. 


\section{Anhang}

\subsection{Sicherheits-, bzw. Ausschlusskriterien tDCS}

Falls einer der folgenden Punkte auf einen Probanden zutraf, war eine Teilnahme an der Studie nicht möglich:

1. Herzschrittmacher

2. Metallimplantate im Kopfbereich (in den Kopfbereich eingesetztes Metall, z.B. Clips nach Operation eines intrazerebralen Aneurysmas (Gefäßaussackung im Bereich der Gehirngefäße), Implantation eine künstlichen Hörschnecke, eventuell auch Metallimplantate (Nägel, Platten, Schrauben) in anderen Körperabschnitten

3. Alter $<18$ oder $>75$ Jahre.

4. Hinweise auf eine chronische oder Residuen (Reste) einer neurologischen Erkrankung (Erkrankung des Nervensystems) in der Vorgeschichte.

5. Intrazerebrale Ischämien (Mangeldurchblutung des Gehirns/Schlaganfall/Blutungen) in der Vorgeschichte.

6. Hinweise auf epileptische Anfälle in der Vorgeschichte.

7. Vorliegen einer schwerwiegenden internistischen (Erkrankung der inneren Organe) oder psychiatrischen (seelische Erkrankung) Vorerkrankung, insbesondere Schizophrenie (seelische Erkrankung mit Trugwahrnehmungen und Denkstörungen) oder Manie (Erkrankung des Gemütes mit gehobener Stimmung).

8. Schwangerschaft oder Stillperiode

9. Medikamenten-, Drogen- und/oder Alkoholabhängigkeit

10. Rezeptive oder globale Aphasie (Störung des Sprachverständnisses bzw. zusätzlich des Sprechens)

11. Teilnahme an einer anderen klinischen Prüfung innerhalb der letzten 8 Wochen 


\subsection{Edingurgh Handedness Inventory (Oldfield 1971)}

Bitte geben Sie in der folgenden Liste mit Tätigkeiten an, welche Hand Sie für die jeweilige Tätigkeit bevorzugen, indem Sie ein + in die entsprechende Spalte setzen. Sollten Sie eine sehr starke Präferenz einer Hand für die jeweilige Tätigkeit verspüren, tragen Sie bitte ++ in die betreffende Spalte ein. Wenn Sie in einem Fall keine Hand bevorzugen, tragen Sie bitte + in beide Spalten ein.

Versuchen Sie bitte, alle Fragen zu beantworten. Lassen Sie nur dann eine Lücke, wenn Sie mit einer Tätigkeit überhaupt keine Erfahrung haben.

$\begin{array}{lll} & \text { rechts } & \text { links } \\ \text { Schreiben } & - & - \\ \text { Zeichnen } & - & - \\ \text { Werfen } & - & - \\ \text { Schere } & - & - \\ \text { Zahnbürste } & - & - \\ \text { Messer (ohne Gabel) } & - & - \\ \text { Löffel } & - & \\ \text { Besen (obere Hand) } & - & \\ \text { Streichholz anzünden (Streichholz) } & - & \\ \text { Schachtel öffnen (Deckel) } & - & \end{array}$

Welchen Fuß bevorzugen Sie zum Schießen?

Welches Auge nutzen Sie, wenn Sie nur eines benutzen würden?

L.Q.:

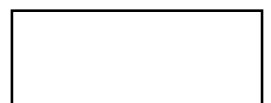

Decile:

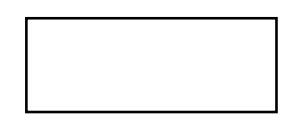




\section{3 Übungen}

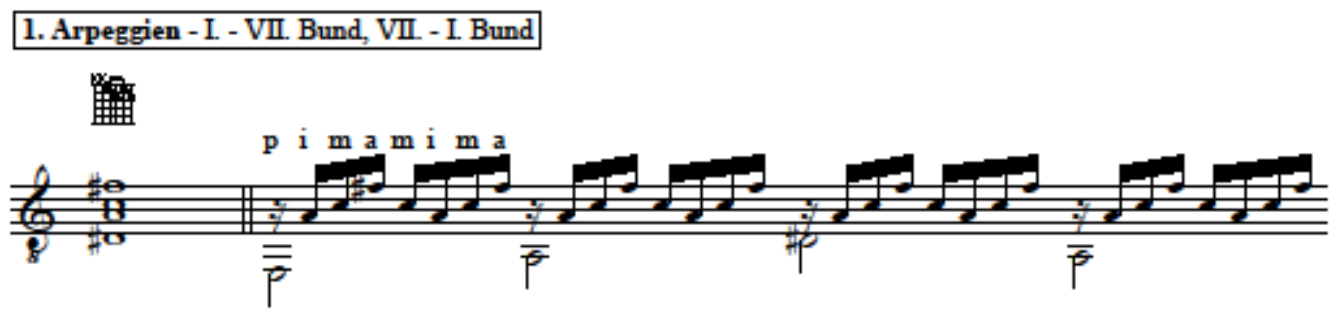

\section{Leere Saiten}

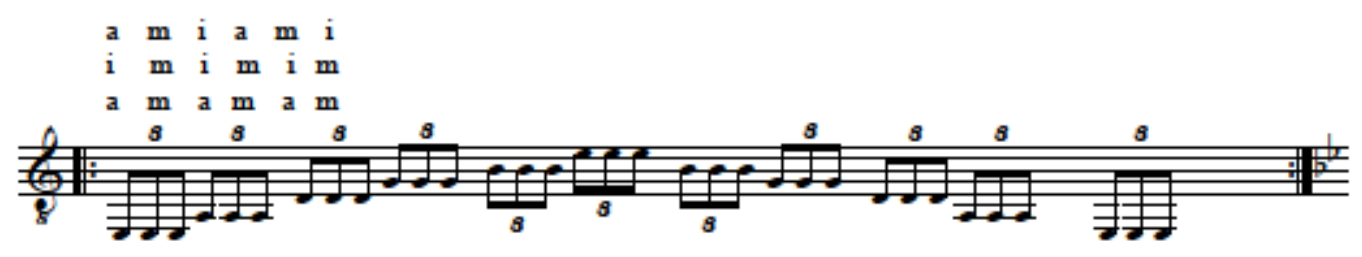

\begin{tabular}{l} 
3. Tonleiter - Bb-Dur, V. Lage (=F\#-Dur-Typ) \\
1. p-i \\
2. $\mathrm{m}-\mathrm{i}$ \\
3. $\mathrm{a}-\mathrm{m}$ \\
\hline
\end{tabular}

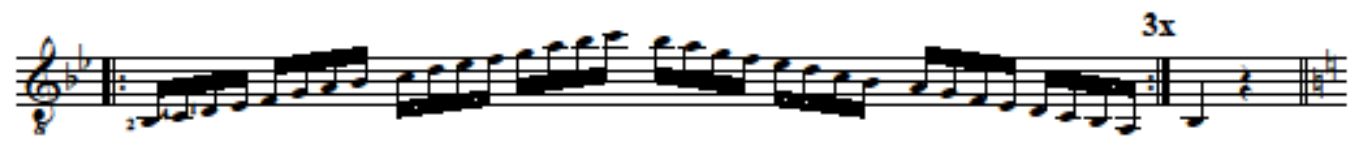

4. Simultananschlag

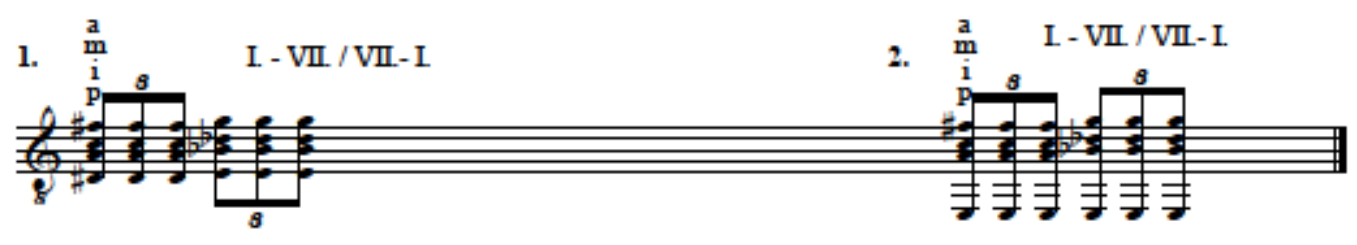




\subsection{STAI Fragebögen (Laux et al. 1981)}

Fragebogen zur Angst als momentaner Zustand (State):

Fragebogen zur Selbstbeschreibung

STAI-G Form X 1

Name

Mädchenname

Vorname Geburtsdatum Alter __Jahre

Beruf Geschlecht $\mathrm{m} / \mathrm{w}$

\begin{tabular}{|c|c|c|c|c|}
\hline $\begin{array}{l}\text { Anleitung: Im folgenden Fragebogen finden Sie eine Reihe von } \\
\text { Feststellungen, mit denen man sich selbst beschreiben kann. Bitte } \\
\text { esen Sie jede Feststellung durch und wählen Sie aus den vier Ant- } \\
\text { worten diejenige aus, die angibt, wie Sie sich jetzt, d.h. in diesem } \\
\text { Moment, fühlen. Kreuzen Sie bitte bei jeder Feststellung die Zahl } \\
\text { unter der von Ihnen gewählten Antwort an. } \\
\text { Es gibt keine richtigen oder falschen Antworten, überlegen Sie bitte } \\
\text { hicht lange und denken Sie daran, diejenige Antwort auszuwählen, } \\
\text { die Ihren augenblicklichen Gefühlszustand am besten beschreibt. }\end{array}$ & 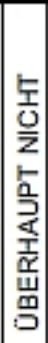 & 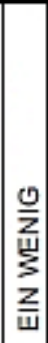 & $\frac{\frac{T}{0}}{\frac{T}{5}}$ & $\frac{\alpha}{\frac{\alpha}{山}}$ \\
\hline 1. Ich bin ruhig & 1 & 2 & 3 & 4 \\
\hline 2. Ich fühle mich geborgen & 1 & 2 & 3 & 4 \\
\hline 3. Ich fühle mich entspannt & 1 & 2 & 3 & 4 \\
\hline 4. Ich bin bekümmert & 1 & 2 & 3 & 4 \\
\hline 5. Ich bin gelöst & 1 & 2 & 3 & 4 \\
\hline 6. Ich bin aufgeregt & 1 & 2 & 3 & 4 \\
\hline 7. Ich bin besorgt, dass etwas schief gehen könnte & 1 & 2 & 3 & 4 \\
\hline 8. Ich fühle mich ausgeruht & 1 & 2 & 3 & 4 \\
\hline 9. Ich bin beunruhigt & 1 & 2 & 3 & 4 \\
\hline 10. Ich fühle mich wohl & 1 & 2 & 3 & 4 \\
\hline 11. Ich fühle mich selbstsicher & 1 & 2 & 3 & 4 \\
\hline 12. Ich bin nervös & 1 & 2 & 3 & 4 \\
\hline 13. Ich bin zappelig & 1 & 2 & 3 & 4 \\
\hline 14. Ich bin verkrampft & 1 & 2 & 3 & 4 \\
\hline 15. Ich bin entspannt & 1 & 2 & 3 & 4 \\
\hline 16. Ich bin zufrieden & 1 & 2 & 3 & 4 \\
\hline 17. Ich bin besorgt & 1 & 2 & 3 & 4 \\
\hline 18. Ich bin überreizt & 1 & 2 & 3 & 4 \\
\hline 19. Ich bin froh & 1 & 2 & 3 & 4 \\
\hline 20. Ich bin vergnügt & 1 & 2 & 3 & 4 \\
\hline
\end{tabular}


Fragebogen zur Änglichkeit als generelle Eigenschaft (Trait):

Fragebogen zur Selbstbeschreibung

STAI-G Form X 2

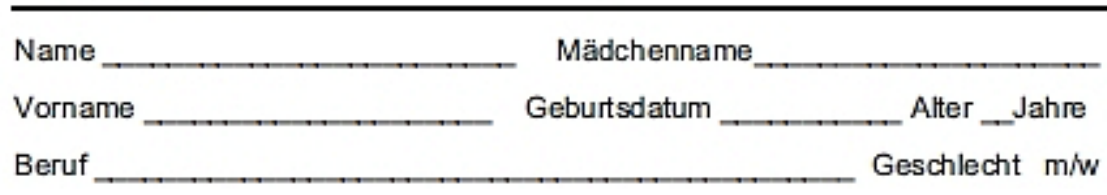

\begin{tabular}{|c|c|c|c|c|}
\hline $\begin{array}{l}\text { Anleitung: Im folgenden Fragebogen finden Sie eine Reihe von } \\
\text { Feststellungen, mit denen man sich selbst beschreiben kann. Bitte } \\
\text { esen Sie jede Feststellung durch und wählen Sie aus den vier Ant- } \\
\text { worten diejenige aus, die angibt, wie Sie sich im allgemeinen fühlen. } \\
\text { Kreuzen Sie bitte bei jeder Feststellung die Zahl unter der von Ihnen } \\
\text { gewählten Antwort an. Es gibt keine richtigen oder falschen Antwor- } \\
\text { en. Überlegen Sie bitte nicht lange und denken Sie daran, diejenige } \\
\text { Antwort auszuwählen, die am besten beschreibt, wie Sie sich im all- } \\
\text { gemeinen fühlen. }\end{array}$ & 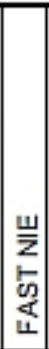 & \begin{tabular}{|l}
$\frac{1}{5}$ \\
$\frac{1}{2}$ \\
$\frac{1}{0}$ \\
$\frac{2}{2}$ \\
$\frac{1}{2}$
\end{tabular} & & 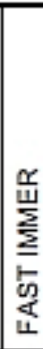 \\
\hline 21. Ich bin vergnügt & 1 & 2 & 3 & 4 \\
\hline 22. Ich werde schnell müde & 1 & 2 & 3 & 4 \\
\hline 23. Mir ist zum Weinen zumute & 1 & 2 & 3 & 4 \\
\hline 24. Ich glaube, mir geht es schlechter als anderen Leuten & 1 & 2 & 3 & 4 \\
\hline $\begin{array}{l}\text { 25. Ich verpasse günstige Gelegenheiten, weil ich mich nicht } \\
\text { schnell genug entscheiden kann }\end{array}$ & 1 & 2 & 3 & 4 \\
\hline 26. Ich fühle mich ausgeruht & 1 & 2 & 3 & 4 \\
\hline 27. Ich bin ruhig und gelassen & 1 & 2 & 3 & 4 \\
\hline $\begin{array}{l}\text { 28. Ich glaube, dass mir meine Schwierigkeiten über den Kopf } \\
\text { wachsen }\end{array}$ & 1 & 2 & 3 & 4 \\
\hline 29. Ich mache mir zu viel Gedanken über unwichtige Dinge & 1 & 2 & 3 & 4 \\
\hline 30. Ich bin glücklich & 1 & 2 & 3 & 4 \\
\hline 31. Ich neige dazu, alles schwer zu nehmen & 1 & 2 & 3 & 4 \\
\hline 32. Mir fehlt es an Selbstvertrauen & 1 & 2 & 3 & 4 \\
\hline 33. Ich fühle mich geborgen & 1 & 2 & 3 & 4 \\
\hline 34. Ich mache mir Sorgen über mögliches Mißgeschick & 1 & 2 & 3 & 4 \\
\hline 35. Ich fühle mich niedergeschlagen & 1 & 2 & 3 & 4 \\
\hline 36. Ich bin zufrieden & 1 & 2 & 3 & 4 \\
\hline $\begin{array}{l}\text { 37. Unwichtige Gedanken gehen mir durch den Kopf und bedrücken } \\
\text { mich }\end{array}$ & 1 & 2 & 3 & 4 \\
\hline $\begin{array}{l}\text { 38. Enttäuschungen nehme ich so schwer, daß ich sie nicht } \\
\text { vergessen kann }\end{array}$ & 1 & 2 & 3 & 4 \\
\hline 39. Ich bin ausgeglichen & 1 & 2 & 3 & 4 \\
\hline $\begin{array}{l}\text { 40. Ich werde nervös und unruhig, wenn ich an meine derzeitigen } \\
\text { Angelegenheiten denke }\end{array}$ & 1 & 2 & 3 & 4 \\
\hline
\end{tabular}




\section{Literaturverzeichnis}

Alexander GE, Crutcher MD (1990): functional architecture of basal ganglia circuits: neural substrates of parallel processing. Trends Neurosci $\underline{13}$, 266-271

Altenmüller E (2003): Focal dystonia: advances in brain imaging and understanding of fine motor control in musicians. Hand Clin 19, 523-538

Altenmüller E, Jabusch HC: Focal hand dystonia in musicians: phenomenology, etiology, and psychological trigger factors. J Hand Ther $2009 \underline{22}$, 144-54

Antal A, Nitsche MA, Kruse W, Tamás ZK, Hoffmann KP, Paulus W (2004a): Direct current stimulation over V5 enhances visuomotor coordination by improving motion perception in humans. J Cogn Neurosci 16, 521-527

Antal A, Nitsche MA, Tamás ZK, Kruse W, Hoffmann KP, Paulus W (2004b): Facilitation of visuo-motor learning by transcranial direct current stimulation of the motor and extrastriate visual areas in humans. Eur J Neurosci $\underline{19}$, 2888-2892

Antal A, Kincses TZ, Nitsche MA, Bartfai O, Paulus W (2004c): Excitability changes induced in the human primary visual cortex by transcranial direct current stimulation. Invest Ophthalmol Vis Sci $\underline{45}, 702-707$

Bara-Jimenez W, Catalan MJ, Hallett M, Gerloff C (1998): abnormal somatosensory homunculus in dystonia of the hand. Ann Neurol $\underline{44}$, 828-831

Bindman LJ, Lippold OCJ, Redfearn JWT (1964): The action of brief polarizing currents on the cerebral cortex of the rat (1) during current flow and (2) in the production of long-lasting after-effects. J Physiol 172, 369-382

Boros K, Poreisz C, Münchau A, Paulus W, Nitsche MA (2008): Premotor transcranial direct current stimulation (tDCS) affects primary motor excitability in humans. Eur J Neurosci 27, $1292-1300$ 
Bortz J, Döring N: Forschungsmethoden und Evaluation für Human- und Sozialwissenschaftler. 4. Auflage; Springer, Heidelberg 2006

Brandfonbrener AG, Robson C (2002): A review of 111 musicians with focal dystonia seen at a performing artist’s clinic 1985-2002. Mov Disord 17, 1135

Byl NN, McKenzie A (2000): Treatment effectiveness for patients with a history of repetitive hand use and focal hand dystonia: a planned, prospective follow-up study. J Hand Ther $\underline{13}$, 289-301

Byl NN, Merzenich MM, Jenkins WM (1996): a primate genesis model of focal dystonia and repetitive strain injury. Neurology $\underline{47}, 508-520$

Candia V, Schäfer T, Taub E, Rau H, Altenmüller E, Rockstroh B, Elbert T (2002): sensory motor retuning: a behavioural treatment for focal hand dystonia of pianists and guitarists. Arch Phys Med Rehabil 83, 1342-1348

Candia V, Wienbruch C, Elbert T, Rockstroh B, Ray W (2003): Effective behavioural treatment of focal hand dystonia in musicians alters somatosensory cortical organization. Proc Natl Acad Sci 100, 7942-7946

Collins KL, Lehmann EM, Patil PG (2010): Deep brain stimulation for movement disorders. Neurobiology of Disease, article in press

Dymond AM, Coger RW, Serafetinides EA (1975): Intracerebral current levels in man during electrosleep therapy. Biol Psychiatry $\underline{10}, 101-104$

Elbert T, Candia V, Altenmüller E, Rau H, Sterr A, Rockstroh B, et al. (1998): alteration of digital representations in somatosensory cortex in focal hand dystonia. Neuroreport $\underline{9}, 3571$ 3575 
Fregni F, Boggio PS, Mansur CG, Wagner T, Ferreira MJL, Lima M, Rigonatti SP, Marcolin MA, Freedman SD, Nitsche MA, Pasual-Leone A (2005): Transcranial direct current stimulation of the unaffected hemisphere in stroke patients. Neuroreport $\underline{16}$, 1551-1555

Gartside IB (1968): Mechanisms of sustained increases of firing rate of neurons in the rat cerebral cortex after polarization: role of protein synthesis. Nature 220, 383-384

Greve W, Wentura D: Wissenschaftliche Beobachtungen: Eine Einführung. Psychologie Verlags Union, Weinheim 1997

Hallett M: Dystonia: abnormal movements result from loss of inhibition. In: Fahn S, Hallett M, DeLong MR (Autoren) “Dystonia 4: Advances in Neurology”, Lippincott Williams \& Wilkins, Philadelphia 2004, 1-9

Hattori Y, Moriwaki A, Hori Y (1990): Biphasic effects of polarizing current on adenosinesensitive generation of cyclic AMP in rat cerebral cortex. Neurosci Lett $\underline{116}$, 320-324

Hummel F, Celnik P, Giraux P, Floel A, Wu WH, Gerloff C, Cohen LG (2005): Effects of non-invasive cortical stimulation on skilled motor function in chronic stroke. Brain $\underline{128}$, 490499

Ikoma K, Samii A, Mercuri B, et al. (1996): abnormal cortical motor excitability in dystonia. Neurology $\underline{46}$, 1371-1376

Islam N, Aftabuddin M, Moriwaki A, Hattori Y, Hori Y (1995): Increase in the calcium level following anodal polarization in the rat brain. Brain Res $\underline{684}$, 206-208

Iyer MB, Mattu U, Grafman J, Lomarev M, Sato S, Wassermann EM (2005): Safety and cognitive effect of frontal DC brain polarization in healthy individuals. Neurology $\underline{64}, 872-$ 875

Jabusch HC, Altenmüller E (2004): Anxiety as an aggravating factor during onset of focal dystonia in musicians. Med Probl Perform Art $\underline{19}$, 81-87 
Jabusch HC, Altenmüller E: Epidemiology, phenomenology and therapy of musician's cramp. In: Altenmüller E, Wiesendanger M, Kesselring J (Autoren) "Music, Motor Control, and the Brain,” Oxford University Press, Oxford 2006, 265-282

Jabusch HC, Vauth H, Altenmüller E (2004a): Quantification of focal dystonia in pianists using scale analysis. Mov Disord $\underline{19}, 171-180$

Jabusch HC, Müller SV, Altenmüller E (2004b): Anxiety in musicians with focal dystonia and those with chronic pain. Mov Disord $\underline{19}$, 1169-1175

Jankovic J, Shale H (1989): Dystonia in musicians. Sem Neurol $\underline{9}$, 131-135

Kuo MF, Paulus W, Nitsche MA (2008): boosting focally-induced brain plasticity by dopamine. Cereb Cortex 18, 648-651

Laux L, Glanzmann P, Schaffner P, Spielberger CD: Das State-Trait-Angstinventar (STAI). Beltz Testgesellschaft, Weinheim 1981

Lederman RJ (1991): Focal dystonia in instrumentalists: clinical features. Med Probl Perform Art $\underline{6}, 132-136$

Liepert J, Miltner WH, Bauder H, Sommer M, Dettmers C, Taub E, Weiller C (1998): Motor cortex plasticity during constrain-induced movement therapy in stroke patients. Neurosci Lett $\underline{250}, 5-8$

Matsumura M, Sawaguchi T, Oishi T, et al. (1991): behavioral deficits induced by local injection of bicuculline and muscimol into the primate motor and premotor cortex. $\mathrm{J}$ Neurophysiol $\underline{65}, 1542-1553$

Matsunaga K, Nitsche MA, Tsuji S, Rothwell J (2004): Effect of transcranial DC sensorimotor cortex stimulation on somatosensory evoked potentials in humans. Clin Neurophysiol $\underline{115}, 456-460$ 
Mink JW (1996): the basal ganglia: focused selection and inhibition of competing motor programs. Prog Neurobiol $\underline{50}$, 381-425

Monte-Silva K, Kuo MF, Liebetanz D, Paulus W, Nitsche MA (2010): shaping the optimal repetition interval for cathodal transcranial direct current stimulation (tDCS). J Neurophysiol $\underline{103}, 1735-1740$

Moriwaki A (1991): Polarizing currents increase noradrenaline-elicited accumulation of cyclic AMP in rat cerebral cortex. Brain Res $\underline{544}$, 248-252

Nitsche MA, Paulus W (2000): Excitability changes induced in the human motor cortex by weak transcranial direct current stimulation. J Physiol 527,633-639

Nitsche MA, Paulus W (2001): sustained excitability elevations induced by transcranial DC motor cortex stimulation in humans. Neurology $\underline{57}, 1899-1901$

Nitsche MA, Fricke K, Henschke U, Schlitterlau A, Liebetanz D, Lang N, Henning S, Tergau F, Paulus W (2003a): Pharmacological modulation of cortical excitability shifts induced by transcranial DC stimulation. J Physiol $\underline{553}$, 293-301

Nitsche MA, Nitsche MS, Klein CC, Tergau F, Rothwell JC, Paulus W (2003b): level of action of cathodal DC polaristion induced inhibition of the human motor cortex. Clin Neurophysiol 114, 600-604

Nitsche MA, Schauenburg A, Lang N, Liebetanz D, Exner C, Paulus W, Tergau F (2003c): Facilitation of implicit motor learning by weak transcranial direct current stimulation of the primary motor cortex in the human. J Cogn Neurosci $\underline{15}$, 619-626

Nitsche MA, Grundey J, Liebetanz D, Lang N, Tergau F, Paulus W (2004a):

Catecholaminergic consolidation of motor cortex neuroplasticity in humans. Cereb Cortex $\underline{14}$, $1240-1245$ 
Nitsche MA, Jaussi W, Liebetanz D, Lang N, Tergau F, Paulus W (2004b): Consolidation of externally induced human motor cortical neuroplasticity by d-cycloserine.

Neuropsychopharmacology $\underline{29}$, 1573-1578

Nitsche MA, Liebetanz D, Schlitterlau A, Henschke U, Fricke K, Lang N, Henning S, Frommann K, Paulus W, Tergau F (2004c): GABAergic modulation of DC-stimulationinduced motor cortex excitability shifts in the human. Eur J Neurosci 19, 2720-2726

Nitsche MA, Niehaus L, Hoffmann KT, Hengst S, Liebetanz D, Paulus W, Meyer BU (2004d): MRI study of human brain exposed to weak direct current stimulation of the frontal cortex. Clin Neurophysiol $\underline{115}$, 2419-2423

Nitsche MA, Lampe C, Antal A, Liebetanz D, Lang N, Tergau F, Paulus W (2006):

Dopaminergic modulation of long-lasting direct current-induced cortical excitability changes in the human motor cortex. Eur J Neurosci 23,1651-1657

Nitsche MA, Doemkes S, Karaköse T, Antal A, Liebetanz D, Lang N, Tergau F, Paulus W (2007): shaping the effects of transcranial direct current stimulation of the human motor cortex. J Neurophysiol 97, 3109-3117

Oldfield RC (1971): The assessment and analysis of handedness: the Edingurgh inventory. Neuropsychologia $\underline{9}$, 97-113

Priori A, Pesenti A, Capellari A, Scarlato G, Barbieri S (2001): limb immobilization for the treatment of focal occupational dystonia. Neurology $\underline{57}$, 405-409

Purpura DP, McMurtry JG (1965): Intracellular activities and evoked potential changes during polarization of motor cortex. J Neurophysiol 28, 166-185

Quartarone A, Rizzo V, Bagnato S, Morgante F, Sant’Angelo A, Romano M, Crupi D, Girlanda P, Rothwell JC, Siebner HR (2005): Homeostatic-like plasticity of the primary motor hand area is impaired in focal hand dystonia. Brain 128,1943-1950 
Ridding MC, Sheean G, Rothwell JC, et al. (1995): changes in the balance between motor cortical excitation and inhibition in focal, task-specific dystonia. J Neurol Neurosurg Psychiatry $\underline{59}$, 493-498

Rogalewski A, Breitenstein C, Nitsche MA, Paulus W, Knecht S (2004): Transcranial direct current stimulation disrupts tactile perception. Eur J Neurosci 20, 313-316

Rosenkranz K, Williamon A, Butler k, Cordivari C, Lees AJ, Rothwell JC (2005):

Pathophysiological differnces between musician’s dystonia and writer's cramp. Brain $\underline{128}$, 918-931

Rothwell JC, Obeso JA, Dy BL, et al. (1983): pathophysiology of dystonias. Adv Neurol $\underline{39}$, 851-863

Ruge D, Tisch S, Limousin P, Hariz MI, Bhatia KP, Quinn N, Zrinzo L, Jahanshahi M, Rothwell JC (2009): Longitudinal effects of deep brain stimulation in the globus pallidus on intracortical GABAergic inhibition and LTP-like plasticty in dystonia. Mov Disord 24 (Supplement), S105-S106

Rush S, Discroll DA (1968): current distribution in the brain from surface electrodes. Anaest Analg Curr Res $\underline{47}$, 717-723

Scholfield CN (1990): Properties of K-currents in unmyelinated presynaptic axons of brain revealed by extracellular polarization. Brain Res $\underline{507,121-128}$

Siebner HR, Lang N, Rizzo V, Nitsche MA, Paulus W, Lemon RN, Rothwell JC (2004):

Preconditioning of low-frequency repetitive transcranial magnetic stimulation with transcranial direct current stimulation: evidence for homeostatic plasticity in the human motor cortex. J Neurosci 24, 3379-3385

Spector JT, Brandfonbrener AG (2005): a new method for quantification of musician’s dystonia: the freqency of abnormal movement scale. Med Probl Perform Art 20, 157-162 
Spector JT, Brandfonbrener AG (2007): methods of evaluation of musician’s dystonia: critique of measurement tools. Mov Disord 22, 309-312

Steinmetz H, Fürst G, Meyer BU (1989): Craniocerebral topography within the international 10-20 system. Electroencephalogr Clin Neurophysiol 72, 499-506

Trepel M: Neuroanatomie: Struktur und Funktion. 3. Auflage; Elsevier GmbH, München 2004

Tubiana R, Chamagne P: Prolonged rehabilitation treatment of musician’s focal dystonia. In: Tubiana R und Amadio PC (Autoren) “Medical problems of the instrumentalist musician”, Martin Dunitz, London 2000, 369-378

Valls-Solé J, Hallett M (1995): modulation of electromyographic activity of wrist flexor and extensor muscles in patients with writer’s cramp. Mov Disord 10, 741-748

Wirtz M, Caspar F: Beurteilerübereinstimmung und Beurteilerreliabilität. Hogrefe, Göttingen 2002

Ziemann U, Lonnecker S, Steinhoff BJ, Paulus W (1996): Effects of antiepileptic drugs on motor cortex excitability in humans: a transcranial magnetic stimulation study. Ann Neurol 40, 367-378 


\section{Danksagung}

Dank sagen möchte ich Herrn Prof. Dr. med. Eckart Altenmüller, Leiter des Institutes für Musikphysiologie und Musikermedizin an der Hochschule für Musik und Theater in Hannover, für seine Ideengebung für dieses Thema und den Entwurf der Studie. Seine überaus kompetente und freundliche Art war mir immer ein Vorbild und Motivation.

Außerdem danken möchte ich Herrn Prof. Dr. med. Walter Paulus als Leiter der Abteilung für klinische Neurophysiologie am Universitätsklinikum Göttingen und besonders Prof. Dr. med. Michael Nitsche für seine Betreuung und sein Wissen über die tDCS, seine häufig geduldigen Erklärungen und zügigen Korrekturen meiner Dissertation.

Für die Betreuung am Institut für Musikphysiologie und Musikermedizin an der Hochschule für Musik und Theater in Hannover danke ich Prof. Dr. med. Hans-Christian Jabusch, mittlerweile Leiter des Institutes für Musikermedizin an der Hochschule für Musik in Dresden, Dr. Dipl.-Psych. Sabine Schneider und insbesondere auch Dipl.-Psych. Franziska Buttkus. 